\title{
Aerobic Acetoxyhydroxylation of Alkenes Cocatalyzed by Organic Nitrite and Palladium
}

\author{
Xian-Min Chen, Xiao-Shan Ning and Yan-Biao Kang* \\ Department of Chemistry, University of Science and Technology of China, Hefei, Anhui 230026, China \\ E-mail: ybkang@ustc.edu.cn
}

\section{Contents}

1. General information

S1

2. General procedures, experiments and data

3. Kinetic study

S18-S28

4. NMR monitoring experiments

5. References

6. NMR spectra

S33-S103

\section{General information}

${ }^{1} \mathrm{H},{ }^{13} \mathrm{C}$ and ${ }^{19} \mathrm{~F}$ NMR spectra were recorded on a Bruker 400 spectrometer. Chemical shifts are reported in $\delta$ units relative to $\mathrm{CDCl}_{3}\left[{ }^{1} \mathrm{H} \delta=7.26,{ }^{13} \mathrm{C} \delta=77.36\right]$ and DMSO- $d_{6}\left[{ }^{1} \mathrm{H} \delta=2.50\right]$. Mass spectra were recorded by the mass spectrometry service at the University of Science and Technology of China. Bis(acetonitrile)dichloropalladium(II), glycial acid and other reagents were commercial available without purification. Acetic anhydride was purified by distillation over $\mathrm{P}_{2} \mathrm{O}_{5}$ under an argon atmosphere.

\section{General procedures}

\subsection{Screening reaction conditions (Table S1)}

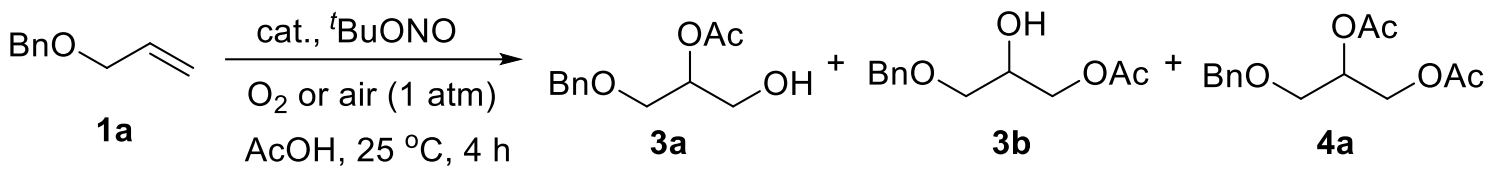

Allyl benzyl ether $(0.5 \mathrm{mmol})$ was weighed into a $50 \mathrm{~mL}$ round-bottomed flask charged with a stir bar, $5 \mathrm{~mL}$ of $\mathrm{AcOH}$ was added to the flask, fallowed with the catalyst $\left[\mathrm{PdCl}_{2}, \mathrm{Pd}(\mathrm{OAc})_{2}, \mathrm{Pd}\left(\mathrm{PPh}_{3}\right)_{2} \mathrm{Cl}_{2}\right.$, 
$\left.\mathrm{Pd}\left(\mathrm{CH}_{3} \mathrm{CN}\right)_{2} \mathrm{ClNO}_{2}, \mathrm{Pd}\left(\mathrm{CH}_{3} \mathrm{CN}\right)_{2}\left(\mathrm{NO}_{2}\right)_{2}, \mathrm{Pd}(\mathrm{PhCN})_{2} \mathrm{Cl}_{2}, \mathrm{Pd}\left(\mathrm{CH}_{3} \mathrm{CN}\right)_{2} \mathrm{Cl}_{2}\right]$ (2.5-7.5 mol \%), and ${ }^{t} \mathrm{BuONO}$ (7.5-20 mol \%) were added at the same time. The reaction vessel was shielded with light using aluminum foil. After stirring for 4 hours with the oil bath and the indoor temperature at $25^{\circ} \mathrm{C}$ under the atmosphere of air or oxygen (balloon), concentrating by a rotary evaporator under reduced pressure, the crude reaction residue was examined by ${ }^{1} \mathrm{H}$ NMR using ethyl 3-phenylpropionate as internal standard and the results were demonstrated in Table S1.

Table S1. Screening reaction conditions ${ }^{a}$

\begin{tabular}{|c|c|c|c|c|c|}
\hline entry & catalyst $(\mathrm{mol} \%)$ & changes & ${ }^{t} \mathrm{BuONO}(\mathrm{mol} \%)$ & $\mathbf{3 a}(\%)^{b}$ & $\mathbf{3 b}(\%)^{b}$ \\
\hline 1 & $\mathrm{Pd}\left(\mathrm{CH}_{3} \mathrm{CN}\right)_{2} \mathrm{Cl}_{2}(2.5)$ & Air & 20 & 15 & 20 \\
\hline 2 & $\mathrm{Pd}\left(\mathrm{CH}_{3} \mathrm{CN}\right)_{2} \mathrm{Cl}_{2}(5)$ & Air & 20 & 17 & 21 \\
\hline 3 & $\mathrm{Pd}\left(\mathrm{CH}_{3} \mathrm{CN}\right)_{2} \mathrm{Cl}_{2}(7.5)$ & Air & 5 & 7 & 16 \\
\hline 4 & $\mathrm{Pd}\left(\mathrm{CH}_{3} \mathrm{CN}\right)_{2} \mathrm{Cl}_{2}(7.5)$ & Air & 7.5 & 10 & 18 \\
\hline 5 & $\mathrm{Pd}\left(\mathrm{CH}_{3} \mathrm{CN}\right)_{2} \mathrm{Cl}_{2}(7.5)$ & Air & 10 & 10 & 24 \\
\hline 6 & $\mathrm{Pd}\left(\mathrm{CH}_{3} \mathrm{CN}\right)_{2} \mathrm{Cl}_{2}(7.5)$ & Air & 15 & 16 & 29 \\
\hline 7 & $\mathrm{Pd}\left(\mathrm{CH}_{3} \mathrm{CN}\right)_{2} \mathrm{Cl}_{2}(7.5)$ & Air & 20 & 21 & 32 \\
\hline 8 & $\mathrm{Pd}\left(\mathrm{CH}_{3} \mathrm{CN}\right)_{2} \mathrm{Cl}_{2}(7.5)$ & with $\mathrm{O}_{2}$ & 20 & 22 & 30 \\
\hline 9 & $\mathrm{Pd}\left(\mathrm{CH}_{3} \mathrm{CN}\right)_{2} \mathrm{Cl}_{2}(7.5)$ & Air & 30 & 24 & 34 \\
\hline 10 & $\mathrm{Pd}\left(\mathrm{CH}_{3} \mathrm{CN}\right) \mathrm{ClNO}_{2}(7.5)$ & Air & - & 17 & 13 \\
\hline 11 & $\mathrm{Pd}\left(\mathrm{CH}_{3} \mathrm{CN}\right)(\mathrm{NO})_{2}(7.5)$ & Air & - & 4 & 8 \\
\hline 12 & $\mathrm{Pd}(\mathrm{PhCN})_{2} \mathrm{Cl}_{2}(7.5)$ & Air & 20 & 13 & 36 \\
\hline 13 & $\mathrm{PdCl}_{2}(7.5)$ & Air & 20 & 15 & 23 \\
\hline 14 & $\mathrm{Pd}\left(\mathrm{PPh}_{3}\right)_{2} \mathrm{Cl}_{2}(7.5)$ & Air & 20 & - & - \\
\hline 15 & $\mathrm{Pd}(\mathrm{OAc})_{2}(7.5)$ & Air & 20 & 3 & 5 \\
\hline 16 & $\mathrm{Pd}(\mathrm{OAc})_{2}(7.5)$ & $\mathrm{O}_{2}, \mathrm{KI}$ & - & - & - \\
\hline 17 & $\mathrm{Pd}\left(\mathrm{CH}_{3} \mathrm{CN}\right)_{2} \mathrm{Cl}_{2}(7.5)$ & under argon & 20 & 2 & 5 \\
\hline 18 & - & Air & 20 & - & - \\
\hline 19 & $\operatorname{Pd}\left(\mathrm{CH}_{3} \mathrm{CN}\right)_{2} \mathrm{Cl}_{2}$ & Air, with light & - & 16 & 30 \\
\hline
\end{tabular}


${ }^{a}$ Conditions: 1a $(0.5 \mathrm{mmol}, 74.1 \mathrm{mg})$, Air/ $\mathrm{O}_{2}$ (1 atm, balloon), ${ }^{t} \mathrm{BuONO}(20 \mathrm{~mol} \%, 10.3 \mathrm{mg})$, catalyst (2.5-7.5 mol \%), AcOH (5 mL), $25{ }^{\circ} \mathrm{C}$, shielded with light. ${ }^{b}$ Determined by ${ }^{1} \mathrm{H}$ NMR (400 MHz), the crude reaction mixture using ethyl 3-phenylpropionate $(0.25 \mathrm{mmol}, 45 \mathrm{mg})$ as internal standard.

\subsection{Preparation of substrates}

(1) but-3-en-1-ylbenzene (1f) ${ }^{1}$

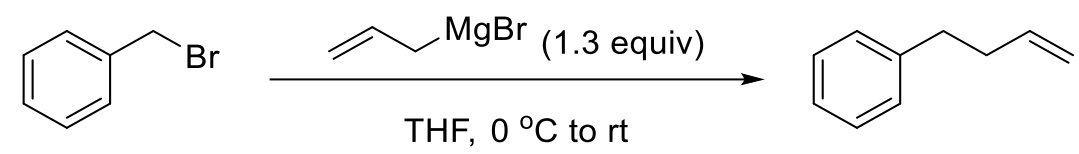

Benzyl bromide (1.71 g, $10 \mathrm{mmol})$ and $20 \mathrm{~mL}$ dry THF were added to a $100 \mathrm{~mL}$ Schlenk bottom, allylmagnesium bromide $(1.0 \mathrm{M}$ in diethyl ether, $15 \mathrm{~mL}, 15 \mathrm{mmol})$ was added dropwise at $0{ }^{\circ} \mathrm{C}$. The resulting suspension was allowed to warm room temperature for $8 \mathrm{~h}$ and then quenched with saturated aqueous $\mathrm{NH}_{4} \mathrm{Cl}$, the aqueous layer was extracted with $\mathrm{CH}_{2} \mathrm{Cl}_{2}$ and the combined organic layers were dried over $\mathrm{Na}_{2} \mathrm{SO}_{4}$, filtered and concentrated by rotary evaporation. The mixture was purified by column chromatography on silica gel $(\mathrm{PE} / \mathrm{EA}=40 / 1)$ to afford a colorless oil $(0.67 \mathrm{~g}, 50 \%) .{ }^{\mathbf{1}} \mathbf{H} \mathbf{N M R}(400 \mathrm{MHz}$, $\left.\mathrm{CDCl}_{3}\right) \delta$ 7.30-7.24 (m, $\left.2 \mathrm{H}\right)$, 7.20-7.16 (m, $\left.3 \mathrm{H}\right)$, 5.91-5.81 (m, $\left.1 \mathrm{H}\right)$, 5.07-5.01 (m, $\left.1 \mathrm{H}\right)$, 4.99-4.96 (m, 1 $\mathrm{H}), 2.71(\mathrm{t}, J=8.0 \mathrm{~Hz}, 2 \mathrm{H}), 2.40-2.34(\mathrm{~m}, 2 \mathrm{H})$.

\section{(2) 1-(but-3-en-1-yl)-4-(trifluoromethyl)benzene (1g) ${ }^{1}$}

Above procedure for but-3-en-1-ylbenzene (1f) was used and the crude product was purified by flash column chromatography on silica gel using petroleum ether as eluent to afford a colorless oil ( $0.73 \mathrm{~g}$, 24\%). ${ }^{1} \mathbf{H}$ NMR (400 MHz, $\left.\mathrm{CDCl}_{3}\right) \delta 7.53(\mathrm{~d}, J=8.4 \mathrm{~Hz}, 2 \mathrm{H}), 7.29(\mathrm{~d}, J=8.0 \mathrm{~Hz}, 2 \mathrm{H})$, 5.88-5.78 (m, 1 H), 5.07-4.98 (m, $2 \mathrm{H}), 2.77(\mathrm{t}, J=7.6 \mathrm{~Hz}, 2 \mathrm{H}), 2.41-2.36(\mathrm{~m}, 2 \mathrm{H}) .{ }^{19} \mathbf{F} \mathbf{N M R}\left(376 \mathrm{MHz}, \mathrm{CDCl}_{3}\right) \delta$ 62.3 .

(3) 1-phenylundec-10-en-1-one (1i)

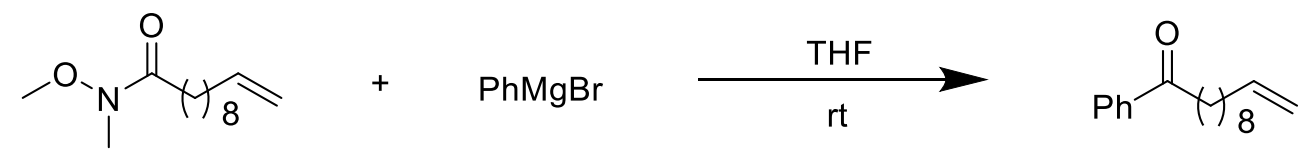

Weinreb amide $(0.68 \mathrm{~g}, 3.0 \mathrm{mmol})$ in dry THF $(20 \mathrm{~mL})$ was added slowly into $\mathrm{PhMgBr}(1.0 \mathrm{M}$ in diethyl 
ether, $3 \mathrm{~mL}, 3.0 \mathrm{mmol}$ ) under $\mathrm{N}_{2}$. After the reaction mixture became clear, it was stirred for $2 \mathrm{~h}$. The reaction was quenched with saturated $\mathrm{NH}_{4} \mathrm{Cl}$ aqueous solution, extracted with ethyl acetate 3 times. The combined organic layers were washed with brine, dried over $\mathrm{Na}_{2} \mathrm{SO}_{4}$, filtered and concentrated in vacuo. The crude product was purified by flash column chromatography on silica gel using petroleum ether and ethyl acetate (50:1) as eluent to afford a colorless oil $(0.64 \mathrm{~g}, 87 \%) .{ }^{1} \mathbf{H} \mathbf{~ N M R}\left(400 \mathrm{MHz}, \mathrm{CDCl}_{3}\right) \delta 7.98$ $7.95(\mathrm{~m}, 2 \mathrm{H}), 7.58-7.53(\mathrm{~m}, 1 \mathrm{H}), 7.48-7.44(\mathrm{~m}, 2 \mathrm{H}), 5.86-5.76(\mathrm{~m}, 1 \mathrm{H}), 5.02-4.91(\mathrm{~m}, 2 \mathrm{H}), 2.96(\mathrm{t}, J=$ 7.6 Hz, $2 \mathrm{H}), 2.07-2.01(\mathrm{~m}, 2 \mathrm{H}), 1.77-1.70(\mathrm{~m}, 2 \mathrm{H}), 1.40-1.30(\mathrm{~m}, 10 \mathrm{H}) .{ }^{13} \mathbf{C ~ N M R}\left(100 \mathrm{MHz}, \mathrm{CDCl}_{3}\right)$ $\delta 200.9,139.5,137.4,133.2,128.9,128.4,114.5,38.9,34.1,29.7$ (two peaks), 29.6, 29.4, 29.2, 24.7. HRMS (ESI) calcd for calcd for $\mathrm{C}_{17} \mathrm{H}_{25} \mathrm{O}[\mathrm{M}+\mathrm{H}]^{+} 245.1900$, found: 245.1897.

\section{(4) Dodec-11-en-2-one (1j) $)^{2}$}

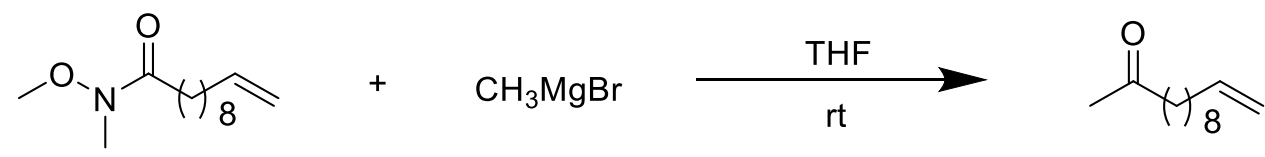

Above procedure for 1-phenylundec-10-en-1-one (1i) was used and the crude product was purified by flash column chromatography on silica gel using petroleum ether and ethyl acetate (50:1) as eluent to afford a colorless oil $(1.73 \mathrm{~g}, 95 \%) .{ }^{1} \mathbf{H}$ NMR $\left(400 \mathrm{MHz}, \mathrm{CDCl}_{3}\right) \delta$ 5.86-5.76 (m, $\left.1 \mathrm{H}\right), 5.01-4.92(\mathrm{~m}, 2$ H), $2.42(\mathrm{t}, J=7.4 \mathrm{~Hz}, 2 \mathrm{H}), 2.13(\mathrm{~s}, 3 \mathrm{H}), 2.04$ (q, $J=7.2 \mathrm{~Hz}, 2 \mathrm{H}), 1.60-1.53(\mathrm{~m}, 2 \mathrm{H}), 1.40-1.34$ (m, 2 $\mathrm{H}), 1.28(\mathrm{~s}, 8 \mathrm{H})$.

\section{(5) $N$-methoxy- $N$-methylundec-10-enamide $(1 \mathrm{k})^{3}$}

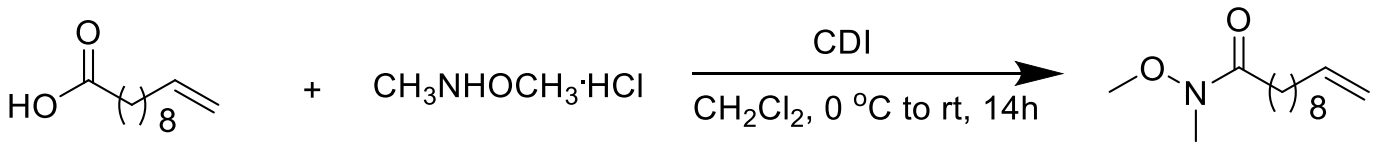

Undec-10-enoic acid $(1.84 \mathrm{~g}, 10.0 \mathrm{mmol})$ was dissolved in $\mathrm{CH}_{2} \mathrm{Cl}_{2}(75 \mathrm{~mL})$ and cooled to $0{ }^{\circ} \mathrm{C}$. carbonyldiimidazole $(1.94 \mathrm{~g}, 12.0 \mathrm{mmol})$ was added and the reaction mixture was stirred at $0{ }^{\circ} \mathrm{C}$ for 0.5 h, then $\mathrm{N}, \mathrm{O}$-dimethylhydroxylamine hydrochloride $(2.44 \mathrm{~g}, 25.0 \mathrm{mmol})$ was added. The reaction mixture was warmed to room temperature and stirred overnight. The resulting suspension was then filtered and the precipitate was washed with $1 \mathrm{M} \mathrm{HCl}$ aqueous solution and brine. The organic phase was dried with $\mathrm{Na}_{2} \mathrm{SO}_{4}$, filtered and concentrated. The crude product was purified by column chromatography on silica gel using petroleum ether and ethyl acetate (10:1) as eluent to afford a colorless oil (1.86 g, 87\%). ${ }^{1} \mathbf{H}$ 
NMR $\left(400 \mathrm{MHz}, \mathrm{CDCl}_{3}\right) \delta$ 5.85-5.75 (m, $\left.1 \mathrm{H}\right)$, 5.00-4.90 (m, $\left.2 \mathrm{H}\right), 3.67(\mathrm{~s}, 3 \mathrm{H}), 3.17(\mathrm{~s}, 3 \mathrm{H}), 2.40(\mathrm{t}, J$ $=7.6 \mathrm{~Hz}, 2 \mathrm{H}), 2.03(\mathrm{q}, J=7.2 \mathrm{~Hz}, 2 \mathrm{H}), 1.65-1.58(\mathrm{~m}, 2 \mathrm{H}), 1.38-1.30(\mathrm{~m}, 10 \mathrm{H})$.

(6) 2-(but-3-en-1-yl)isoindoline-1,3-dione (11) ${ }^{4}$<smiles>C=CCCBr</smiles><smiles>CCC(C)C(C)C</smiles><smiles>C=CCN1C(=O)c2ccccc2C1=O</smiles>

To a mixture of phthalimide $(1.77 \mathrm{~g}, 12 \mathrm{mmol})$ and potassium carbonate $(4.14 \mathrm{~g}, 30 \mathrm{mmol})$ in acetonitrile (25 mL), 4-bromo-1-butene $(1.0 \mathrm{~mL}, 10 \mathrm{mmol})$ was added at room temperature. The resulting reaction mixture was stirred at $90{ }^{\circ} \mathrm{C}$ for $13 \mathrm{~h}$. After the reaction was completed, the reaction mixture was filtered through a plug of Celite. Concentrated in vacuo, and the crude reaction mixture was purified by column chromatography on silica gel using petroleum ether and ethyl acetate (20:1) as eluent to afford a white solid (1.81 g, 90\%). m.p.: 52-53 ${ }^{\circ} \mathrm{C} .{ }^{1} \mathbf{H}$ NMR (400 MHz, $\left.\mathrm{CDCl}_{3}\right) \delta$ 7.84-7.81 (m, $\left.2 \mathrm{H}\right)$, 7.72-7.68 (m, 2 H), 5.83-5.73 (m, 1 H), 5.08-5.00 (m, 2 H), 3.76 (t, $J=7.2 \mathrm{~Hz}, 2 \mathrm{H}), 2.44$ (dd, $J=14.0,7.2 \mathrm{~Hz}, 2 \mathrm{H})$.

(7) Dec-9-en-1-yl 4-methylbenzenesulfonate (1m) ${ }^{5}$

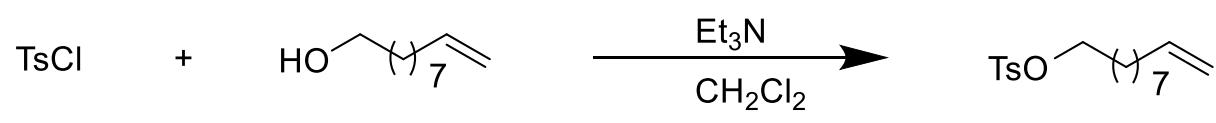

Dec-9-en-1-ol (1.56 g, $10.0 \mathrm{mmol})$ and tosyl chloride $(2.1 \mathrm{~g}, 11.0 \mathrm{mmol})$ was dissolved in $\mathrm{CH}_{2} \mathrm{Cl}_{2}$ (45 $\mathrm{mL})$, triethylamine $(1.11 \mathrm{~g}, 11.0 \mathrm{mmol})$ was added to the reaction. The mixture was stirred at $50{ }^{\circ} \mathrm{C}$ for 4 $\mathrm{h}$ (monitored by TLC). Then the reaction solution washed with water $(3 \times 50 \mathrm{~mL})$, dried with $\mathrm{Na}_{2} \mathrm{SO}_{4}$, filtered and concentrated. The residue was purified by a shot flash column chromatography on silica gel using petroleum ether and ethyl acetate (40:1) as eluent to afford a colorless oil (2.63 g, 85\%). ${ }^{1} \mathbf{H}$ NMR $\left(400 \mathrm{MHz}, \mathrm{CDCl}_{3}\right) \delta 7.79(\mathrm{~d}, J=8.4 \mathrm{~Hz}, 2 \mathrm{H}), 7.34(\mathrm{~d}, J=8.4 \mathrm{~Hz}, 2 \mathrm{H}), 5.85-5.75(\mathrm{~m}, 1 \mathrm{H}), 5.01-4.91$ (m, $2 \mathrm{H}), 4.02(\mathrm{t}, J=6.6 \mathrm{~Hz}, 2 \mathrm{H}), 2.45(\mathrm{~s}, 3 \mathrm{H}), 2.05-2.00(\mathrm{~m}, 2 \mathrm{H}), 1.66-1.60(\mathrm{~m}, 2 \mathrm{H}), 1.36-1.23(\mathrm{~m}, 10$ $\mathrm{H})$.

(8) Dec-9-en-1-yl methanesulfonate (1n) ${ }^{6}$

$$
\mathrm{MsCl}+\mathrm{HO}^{\mathrm{H}} \widehat{\mathrm{HCM}} \rightarrow \frac{\mathrm{Et}_{3} \mathrm{~N}}{\mathrm{DCM}} \rightarrow \mathrm{MsO}_{7}
$$


Above procedure for dec-9-en-1-yl 4-methylbenzenesulfonate (1m) was used. The residue was purified by a shot flash column chromatography on silica gel using petroleum ether and ethyl acetate (10:1) as eluent to afford a colorless oil $(1.85 \mathrm{~g}, 79 \%) .{ }^{1} \mathbf{H}$ NMR $\left(400 \mathrm{MHz}, \mathrm{CDCl}_{3}\right) \delta 5.86-5.76(\mathrm{~m}, 1 \mathrm{H}), 5.02-$ $4.91(\mathrm{~m}, 2 \mathrm{H}), 4.22(\mathrm{t}, J=6.6 \mathrm{~Hz}, 2 \mathrm{H}), 3.00$ (s, $3 \mathrm{H}), 2.07-2.01(\mathrm{~m}, 2 \mathrm{H}), 1.78-1.71(\mathrm{~m}, 2 \mathrm{H}), 1.41-1.28$ $(\mathrm{m}, 10 \mathrm{H})$.

\section{(9) (but-3-en-1-yloxy)(tert-butyl)diphenylsilane (10) ${ }^{7}$}

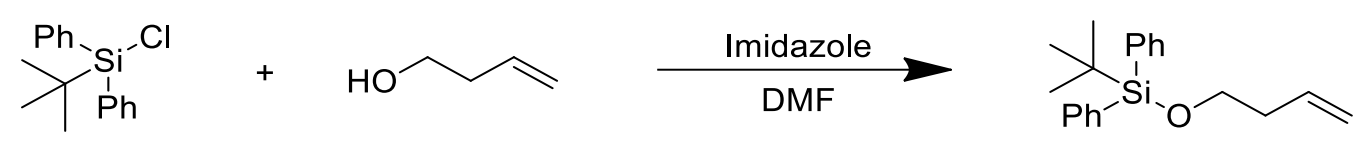

But-3-en-1-ol (0.36 g, $5.0 \mathrm{mmol})$ was dissolved in DMF (15 mL), imidzole (1.23 g, $14.0 \mathrm{mmol})$ and tertbutylchlorodiphenylsilane $(1.92 \mathrm{~g}, 7.0 \mathrm{mmol})$ were added and the reaction mixture was stirred at $50{ }^{\circ} \mathrm{C}$ for $24 \mathrm{~h}$. Then the suspension was washed with $1 \mathrm{M} \mathrm{HCl}$ solution and extracted with $\mathrm{Et}_{2} \mathrm{O}$, the organic phase was washed with brine and dried over $\mathrm{Na}_{2} \mathrm{SO}_{4}$, filtered and concentrated. The residue was purified by a shot flash column chromatography on silica gel using petroleum ether and ethyl acetate (50:1) as eluent to afford a colorless oil (1.32 g, 90\%). ${ }^{\mathbf{1}} \mathbf{H}$ NMR (400 MHz, $\left.\mathrm{CDCl}_{3}\right) \delta$ 7.70-7.67 (m, $\left.4 \mathrm{H}\right), 7.45$ 7.37 (m, $6 \mathrm{H}), 5.90-5.80(\mathrm{~m}, 1 \mathrm{H}), 5.09-5.01(\mathrm{~m}, 2 \mathrm{H}), 3.72(\mathrm{t}, J=6.8 \mathrm{~Hz}, 2 \mathrm{H}), 2.33(\mathrm{dd}, J=13.4,6.6 \mathrm{~Hz}$, $2 \mathrm{H}), 1.06(\mathrm{~s}, 9 \mathrm{H})$.

\subsection{General procedure for dihydroxylation of alkenes}

The alkene substrates $(0.5 \mathrm{mmol})$ was weighed into a $50 \mathrm{~mL}$ round-bottomed flask charged with a stir bar, $5 \mathrm{~mL}$ of $\mathrm{AcOH}$ was added to the flask, fallowed with $\mathrm{Pd}\left(\mathrm{CH}_{3} \mathrm{CN}\right)_{2} \mathrm{Cl}_{2}(7.5 \mathrm{~mol} \%, 9.7 \mathrm{mg})$ and ${ }^{t}$ BuONO (20 mol \%, $10.3 \mathrm{mg}$ ) were added at the same time. The reaction vessel was shielded with light using aluminum foil. After stirring for the setted time with the oil bath and the indoor temperature at 25 ${ }^{\circ} \mathrm{C}$ under the atmosphere of air or oxygen (balloon), The solvent was removed under reduced pressure, then $\mathrm{MeOH}(5 \mathrm{~mL})$ and $\mathrm{K}_{2} \mathrm{CO}_{3}(2.0 \mathrm{mmol})$ was added to the flask. The reaction was then allowed to stir at $-10^{\circ} \mathrm{C}$ to $25^{\circ} \mathrm{C}$ for $1-8 \mathrm{~h}$. $\mathrm{MeOH}$ was removed under reduced pressure at reaction temperature and the crude mixture was purified by silica gel chromatography to afford the corresponding products.

\subsection{General procedure for diacetoxylation of alkenes}


$\mathrm{Pd}\left(\mathrm{CH}_{3} \mathrm{CN}\right)_{2} \mathrm{Cl}_{2}(7.5 \mathrm{~mol} \%, 9.7 \mathrm{mg})$ was weighed directly into a $50 \mathrm{~mL}$ round-bottomed flask, charged with a stir bar and dried under high vacuum for 15 mins, purge oxygen or air 3 times. Under an atmosphere of oxygen or air ( $1 \mathrm{~atm}$, balloon) $5 \mathrm{~mL}$ of $\mathrm{AcOH}$ and ${ }^{t} \mathrm{BuONO}(20 \mathrm{~mol} \%, 10.3 \mathrm{mg})$ were added and stirred at the oil bath and the indoor temperature at $25^{\circ} \mathrm{C}$. Alkene $(0.5 \mathrm{mmol})$ was then added and the reaction vessel was shielded with light using aluminum foil, the resulting reaction mixture was monitored by TLC. After completion, $\mathrm{AcOH}$ was removed under reduced pressure, then $2 \mathrm{~mL} \mathrm{Ac}_{2} \mathrm{O}$ was added to the flask, the reaction mixture was then allowed to stir at $45^{\circ} \mathrm{C}$ for $4 \mathrm{~h}$. After completion, the solvent was removed under reduced pressure and the crude mixture was purified by chromatography on silica gel to afford the corresponding products.

$\underbrace{\mathrm{OH}}_{3-\left(\text { benzyloxy)propane-1,2-diol (2a) }{ }^{8}\right.}$

Prepared according to the general procedure $2.3,25^{\circ} \mathrm{C}, 8 \mathrm{~h}$, purified by flash column chromatography $(\mathrm{PE} / \mathrm{EA}=2 / 1)$, colorless oil $(49.9 \mathrm{mg}, 55 \%) .{ }^{1} \mathbf{H}$ NMR $\left(400 \mathrm{MHz}, \mathrm{CDCl}_{3}\right) \delta$ 7.38-7.28 (m, $\left.5 \mathrm{H}\right), 4.55(\mathrm{~s}$, $2 \mathrm{H}), 3.89(\mathrm{~s}, 1 \mathrm{H}), 3.70(\mathrm{dd}, J=11.2,3.2 \mathrm{~Hz}, 1 \mathrm{H}), 3.62(\mathrm{dd}, J=11.6,5.6 \mathrm{~Hz}, 1 \mathrm{H}), 3.59-3.51(\mathrm{~m}, 2 \mathrm{H})$, 2.89 (br s, $1 \mathrm{H}), 244$ (br s, $1 \mathrm{H})$.<smiles>CCC(O)CO</smiles>

\section{Hexane-1,2-diol (2b) ${ }^{8}$}

Prepared according to the general procedure $2.3,25{ }^{\circ} \mathrm{C} 8 \mathrm{~h}$, purified by flash column chromatography $(\mathrm{PE} / \mathrm{EA}=2 / 1)$, colorless oil (42.8 mg, 72\%). ${ }^{1} \mathbf{H}$ NMR $\left(400 \mathrm{MHz}, \mathrm{CDCl}_{3}\right) \delta 3.73-3.68(\mathrm{~m}, 1 \mathrm{H}), 3.65(\mathrm{dd}$, $J=11.0,3.0 \mathrm{~Hz}, 1 \mathrm{H}), 3.43(\mathrm{dd}, J=11.2,7.6 \mathrm{~Hz}, 1 \mathrm{H}), 2.40(\mathrm{br} \mathrm{s}, 2 \mathrm{H}), 1.44-1.28(\mathrm{~m}, 6 \mathrm{H}), 0.91(\mathrm{t}, J=$ $7.0 \mathrm{~Hz}, 3 \mathrm{H})$.<smiles>CCCC(O)CO</smiles>

\section{Butane-1,2-diol (2c) ${ }^{9}$}

Prepared according to the general procedure $2.3,25{ }^{\circ} \mathrm{C} 8 \mathrm{~h}$, purified by flash column chromatography $(\mathrm{PE} / \mathrm{EA}=2 / 1)$, colorless oil (55.8 mg, 76\%). ${ }^{1} \mathbf{H} \mathbf{~ N M R}\left(400 \mathrm{MHz}, \mathrm{CDCl}_{3}\right) \delta 3.72-3.65(\mathrm{~m}, 2 \mathrm{H}), 3.44(\mathrm{dd}$, $J=10.2,8.6 \mathrm{~Hz}, 1 \mathrm{H}), 2.15($ br s, $1 \mathrm{H}), 2.05(\mathrm{br} \mathrm{s}, 1 \mathrm{H}), 1.45-1.29(\mathrm{~m}, 10 \mathrm{H}), 0.88(\mathrm{t}, J=6.6 \mathrm{~Hz}, 3 \mathrm{H})$. 
$\mathrm{Me}_{9}^{\mathrm{OH}} \mathrm{OH}$

Dodecane-1,2-diol (2d) ${ }^{10}$

Prepared according to the general procedure $2.3,25{ }^{\circ} \mathrm{C} 8 \mathrm{~h}$, purified by flash column chromatography (PE/EA = 2/1), white solid (71.8 mg, 71\%). m.p.: 52-54 ${ }^{\circ} \mathrm{C} .{ }^{1} \mathbf{H}$ NMR (400 MHz, $\mathrm{CDCl}_{3}$,) $\delta 3.72-3.65$ (m, $2 \mathrm{H}), 3.46-3.42(\mathrm{~m}, 1 \mathrm{H}), 2.03$ (br s, $1 \mathrm{H}), 1.91$ (br s, $1 \mathrm{H}), 1.48-1.26(\mathrm{~m}, 18 \mathrm{H}), 0.88(\mathrm{t}, J=6.8 \mathrm{~Hz}, 3 \mathrm{H})$.<smiles>CC(O)CBr</smiles>

\section{6-bromohexane-1,2-diol (2e)}

Prepared according to the general procedure $2.3,0{ }^{\circ} \mathrm{C} 8 \mathrm{~h}$, purified by flash column chromatography $(\mathrm{PE} / \mathrm{EA}=2 / 1)$, colorless oil (84.4 mg, 86\%). ${ }^{1} \mathbf{H}$ NMR $\left(400 \mathrm{MHz}, \mathrm{CDCl}_{3}\right) \delta$ 3.76-3.70 (m, $\left.1 \mathrm{H}\right), 3.67(\mathrm{dd}$, $J=10.8,2.8 \mathrm{~Hz}, 1 \mathrm{H}), 3.48-3.41$ (m, $3 \mathrm{H}), 2.15$ (br s, $2 \mathrm{H}), 1.94-1.86$ (m, $2 \mathrm{H}), 1.68-1.44(\mathrm{~m}, 4 \mathrm{H}) .{ }^{13} \mathrm{C}$ NMR $\left(100 \mathrm{MHz}, \mathrm{CDCl}_{3}\right) \delta 72.3,67.1,33.9,32.9,32.5,24.5$. HRMS (ESI) calcd for $\mathrm{C}_{6} \mathrm{H}_{13} \mathrm{O}_{2} \mathrm{BrNa}$ $[\mathrm{M}+\mathrm{Na}]^{+}$218.9997, found: 218.9992 .

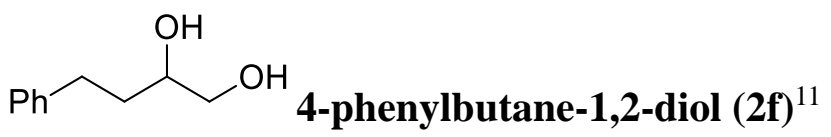

Prepared according to the general procedure $2.3,25^{\circ} \mathrm{C}, 8 \mathrm{~h}$, purified by flash column chromatography $(\mathrm{PE} / \mathrm{EA}=2 / 1)$, colorless oil $(66.4 \mathrm{mg}, 80 \%)$. ${ }^{1} \mathbf{H}$ NMR (400 MHz, $\left.\mathrm{CDCl}_{3}\right) \delta$ 7.26-7.22 (m, $\left.2 \mathrm{H}\right), 7.17$ $7.13(\mathrm{~m}, 3 \mathrm{H}), 3.71-3.66(\mathrm{~m}, 1 \mathrm{H}), 3.61(\mathrm{dd}, J=11.0,3.0 \mathrm{~Hz}, 1 \mathrm{H}), 3.42(\mathrm{dd}, J=11.2,7.6 \mathrm{~Hz}, 1 \mathrm{H}), 2.81-$ 2.73 (m, $1 \mathrm{H}), 2.69-2.62$ (m, $1 \mathrm{H}), 2.29$ (br s, $1 \mathrm{H}), 2.11$ (br s, $1 \mathrm{H}), 1.79-1.65$ (m, $2 \mathrm{H})$.<smiles>OCC(O)CCc1ccc(C(F)(F)F)cc1</smiles>

4-(4-(trifluoromethyl)phenyl)butane-1,2-diol (2g)

Prepared according to the general procedure $2.3,25{ }^{\circ} \mathrm{C}, 8 \mathrm{~h}$, purified by flash column chromatography (PE/EA = 2/1), colorless oil $(98.7 \mathrm{mg}, 84 \%) .{ }^{1} \mathbf{H} \mathbf{N M R}\left(400 \mathrm{MHz}, \mathrm{CDCl}_{3}\right) \delta 7.53(\mathrm{~d}, J=8.0 \mathrm{~Hz}, 2 \mathrm{H})$, $7.31(\mathrm{~d}, J=7.6 \mathrm{~Hz}, 2 \mathrm{H}), 3.74-3.69$ (m, $1 \mathrm{H}), 3.66$ (dd, $J=10.8,3.2 \mathrm{~Hz}, 1 \mathrm{H}), 3.47$ (dd, $J=10.8,7.6 \mathrm{~Hz}$, $1 \mathrm{H}), 2.91-2.84(\mathrm{~m}, 1 \mathrm{H}), 2.79-2.71(\mathrm{~m}, 1 \mathrm{H}), 2.39$ (br s, $2 \mathrm{H}), 1.83-1.69$ (m, $2 \mathrm{H}) .{ }^{13} \mathbf{C} \mathbf{N M R}(100 \mathrm{MHz}$, $\left.\mathrm{CDCl}_{3}\right) \delta 146.1(\mathrm{~d}, J=1.5 \mathrm{~Hz}), 129.0,128.7(\mathrm{q}, J=32.1), 125.7(\mathrm{q}, J=3.5 \mathrm{~Hz}), 71.6,67.0,34.6,31.9$. ${ }^{19} \mathbf{F}$ NMR (376 MHz, $\left.\mathrm{CDCl}_{3}\right) \delta$-62.4. HRMS (ESI) calcd for $\mathrm{C}_{11} \mathrm{H}_{14} \mathrm{~F}_{3} \mathrm{O}_{2}[\mathrm{M}+\mathrm{H}]^{+}$235.0940, found:235.0938. 


\section{0,11-dihydroxyundecanoic acid $(\mathbf{2 h})^{12}$}

Prepared according to the general procedure 2.3, $10 \mathrm{~mL} \mathrm{AcOH}, 25^{\circ} \mathrm{C}, 8 \mathrm{~h}$ purified by flash column chromatography (PE/EA = 1/1), white solid (91.4 mg, 84\%). m.p.: $64{ }^{\circ} \mathrm{C} .{ }^{1} \mathbf{H}$ NMR $(400 \mathrm{MHz}$, DMSOd6) $\delta 11.95(\mathrm{~s}, 1 \mathrm{H}), 4.38(\mathrm{~s}, 1 \mathrm{H}), 4.30(\mathrm{~s}, 1 \mathrm{H}), 3.40-3.32(\mathrm{~m}, 3 \mathrm{H}), 3.27-3.19(\mathrm{~m}, 2 \mathrm{H}), 2.18(\mathrm{t}, J=7.4$ $\mathrm{Hz}, 2 \mathrm{H}), 1.48$ (t, $J=6.6 \mathrm{~Hz}, 2 \mathrm{H}), 1.43-1.33$ (m, $2 \mathrm{H}), 1.31-1.14$ (m, $8 \mathrm{H})$.<smiles>O=C(NC(O)CO)c1ccccc1</smiles>

\section{0,11-dihydroxy-1-phenylundecan-1-one (2i)}

Prepared according to the general procedure $2.3,25^{\circ} \mathrm{C}, 8 \mathrm{~h}$, purified by flash column chromatography $(\mathrm{PE} / \mathrm{EA}=2 / 1)$, white solid $(100.4 \mathrm{mg}, 72 \%)$. m.p.: 68-69 ${ }^{\circ} \mathrm{C} .{ }^{\mathbf{1}} \mathbf{H} \mathbf{N M R}\left(400 \mathrm{MHz}, \mathrm{CDCl}_{3}\right) \delta$ 7.97-7.95 (m, $2 \mathrm{H}), 7.58-7.54(\mathrm{~m}, 1 \mathrm{H}), 7.48-7.44(\mathrm{~m}, 2 \mathrm{H}), 3.73-3.64(\mathrm{~m}, 2 \mathrm{H}), 3.44(\mathrm{dd}, J=10.8,7.6 \mathrm{~Hz}, 1 \mathrm{H})$, $2.97(\mathrm{t}, J=7.2 \mathrm{~Hz}, 2 \mathrm{H}), 2.11$ (br s, $1 \mathrm{H}), 1.99$ (br s, $1 \mathrm{H}), 1.77-1.70$ (m, $2 \mathrm{H}), 1.44-1.32(\mathrm{~m}, 12 \mathrm{H}) .{ }^{13} \mathrm{C}$ NMR $\left(100 \mathrm{MHz}, \mathrm{CDCl}_{3}\right) \delta 201.0,137.4,133.3,128.9,128.4,72.6,67.2,38.9,33.5,29.9,29.7$ (two peaks), 29.6, 25.8, 24.6. HRMS (ESI) calcd for $\mathrm{C}_{17} \mathrm{H}_{26} \mathrm{O}_{3} \mathrm{Na}[\mathrm{M}+\mathrm{Na}]^{+} 301.1780$, found: 301.1781.<smiles>CC(=O)C(O)CO</smiles>

\section{1,12-dihydroxydodecan-2-one (2j)}

Prepared according to the general procedure $2.3,25{ }^{\circ} \mathrm{C}, 8 \mathrm{~h}$, purified by flash column chromatography (PE/EA = 2/1), white solid (75.0 mg, 69\%). m.p.: 58-59 ${ }^{\circ} \mathrm{C} .{ }^{1} \mathbf{H} \mathbf{~ N M R}\left(400 \mathrm{MHz}, \mathrm{CDCl}_{3}\right) \delta 3.71-3.65$ (m, 2 H), 3.46-3.41 (m, $1 \mathrm{H}), 2.42$ (t, $J=7.4 \mathrm{~Hz}, 2 \mathrm{H}), 2.14$ (s, $3 \mathrm{H}), 2.09$ (br s, $1 \mathrm{H}), 1.96$ (br s, $1 \mathrm{H}), 1.60$ $1.54(\mathrm{~m}, 2 \mathrm{H}), 1.43-1.29$ (m, 12 H). ${ }^{13} \mathbf{C} \mathbf{N M R}\left(100 \mathrm{MHz}, \mathrm{CDCl}_{3}\right) \delta 209.9,72.6,67.2,44.1,33.5,30.2$, 29.8, 29.6 (two peaks), 29.4, 25.8, 24.1. HRMS (ESI) calcd for $\mathrm{C}_{12} \mathrm{H}_{24} \mathrm{O}_{3} \mathrm{Na}[\mathrm{M}+\mathrm{Na}]^{+}$239.1623, found: 239.1624 .<smiles>CON(C)C(=O)[Hg]C(O)CO</smiles>

\section{0,11-dihydroxy- $N$-methoxy- $N$-methylundecanamide (2k)}

Prepared according to the general procedure $2.3,25{ }^{\circ} \mathrm{C}, 8 \mathrm{~h}$, purified by flash column chromatography $(\mathrm{PE} / \mathrm{EA}=1 / 1)$, colorless oil $(112.6 \mathrm{mg}, 86 \%) .{ }^{1} \mathbf{H}$ NMR $\left(400 \mathrm{MHz}, \mathrm{CDCl}_{3}\right) \delta 3.66(\mathrm{~s}, 4 \mathrm{H}), 3.61(\mathrm{dd}, J=$ 
11.0, 2.6 Hz, $1 \mathrm{H}), 3.39$ (dd, $J=11.0,7.8 \mathrm{~Hz}, 1 \mathrm{H}), 3.16$ (s, $3 \mathrm{H}), 2.94$ (br s, $2 \mathrm{H}), 2.39$ (t, $J=7.2 \mathrm{~Hz}, 2$ H), $1.59(\mathrm{t}, J=7.2 \mathrm{~Hz}, 2 \mathrm{H}), 1.40-1.28(\mathrm{~m}, 12 \mathrm{H}) .{ }^{13} \mathbf{C} \mathbf{N M R}\left(100 \mathrm{MHz}, \mathrm{CDCl}_{3}\right) \delta 175.2,72.6,67.1,61.5$, 33.4, 32.5, 32.1, 29.8, 29.6 (two peaks), 29.5, 25.8, 24.9. HRMS (ESI) calcd for $\mathrm{C}_{13} \mathrm{H}_{27} \mathrm{NO}_{4} \mathrm{Na}[\mathrm{M}+\mathrm{Na}]^{+}$ 284.1838, found: 284.1841 .

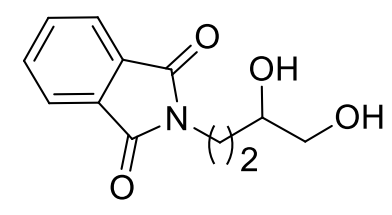

\section{2-(3,4-dihydroxybutyl)isoindoline-1,3-dione (2I)}

Prepared according to the general procedure $2.3,0{ }^{\circ} \mathrm{C} 1 \mathrm{~h}$, purified by flash column chromatography (PE/EA = 1/1), white solid (58.7 mg, 50\%). m.p.: $65^{\circ} \mathrm{C}^{\mathbf{1}}{ }^{\mathbf{H}} \mathbf{N M R}\left(400 \mathrm{MHz}, \mathrm{CDCl}_{3}\right) \delta$ 7.88-7.84 (m, 2 H), 7.76-7.73 (m, 2 H), 3.89 (dd, J = 7.6, 4.8 Hz, 2 H), 3.67-3.58 (m, 2 H), 3.52-3.47 (m, 1 H), 3.30 (br s, $1 \mathrm{H}), 2.16$ (br s, $1 \mathrm{H}), 1.79-1.68$ (m, $2 \mathrm{H}) .{ }^{13} \mathbf{C ~ N M R}\left(100 \mathrm{MHz}, \mathrm{CDCl}_{3}\right) \delta 169.2,134.5,132.3,123.7,69.5$, 66.7, 34.7, 32.3. HRMS (ESI) calcd for $\mathrm{C}_{12} \mathrm{H}_{13} \mathrm{NO}_{4} \mathrm{Na}[\mathrm{M}+\mathrm{Na}]^{+} 258.0742$, found: 258.0741 .<smiles>OC[14CH2]C[14CH2]O</smiles>

\section{9,10-dihydroxydecyl 4-methylbenzenesulfonate (2m)}

Prepared according to the general procedure $2.3,0{ }^{\circ} \mathrm{C} 8 \mathrm{~h}$, purified by flash column chromatography (PE/EA = 2/1), colorless oil (127.3 mg, 74\%). ${ }^{1} \mathbf{H} \mathbf{N M R}\left(400 \mathrm{MHz}, \mathrm{CDCl}_{3}\right) \delta 7.78(\mathrm{~d}, J=8.4 \mathrm{~Hz}, 2 \mathrm{H})$, $7.34(\mathrm{~d}, J=8.0 \mathrm{~Hz}, 2 \mathrm{H}), 4.01(\mathrm{t}, J=6.4 \mathrm{~Hz}, 2 \mathrm{H}), 3.72-3.62(\mathrm{~m}, 2 \mathrm{H}), 3.42(\mathrm{dd}, J=11.0,7.8 \mathrm{~Hz}, 1 \mathrm{H})$, 2.44 (s, 3 H), 2.31 (br s, 2 H), 1.66-1.59 (m, 2 H), 1.46-1.24 (m, 12 H). $\left.{ }^{13} \mathbf{C ~ N M R ~ ( 1 0 0 ~ M H z , ~ C D C l ~} 3\right) \delta$ 145.0, 133.6, 130.1, 128.2, 72.6, 71.0, 67.2, 33.4, 29.7, 29.5, 29.1, 25.7, 25.6, 22.0. HRMS (ESI) calcd for $\mathrm{C}_{17} \mathrm{H}_{28} \mathrm{O} 5 \mathrm{SNa}[\mathrm{M}+\mathrm{Na}]^{+} 367.1555$, found: 367.1558 .<smiles>COC[Al]C(O)CO</smiles>

\section{9,10-dihydroxydecyl methanesulfonate (2n)}

Prepared according to the general procedure $2.3,-10{ }^{\circ} \mathrm{C} 6 \mathrm{~h}$, purified by flash column chromatography $(\mathrm{PE} / \mathrm{EA}=1 / 1)$, white solid $(105.0 \mathrm{mg}, 78 \%)$. m.p.: 60-61 ${ }^{\circ} \mathrm{C} .{ }^{1} \mathbf{H} \mathbf{N M R}\left(400 \mathrm{MHz}, \mathrm{CDCl}_{3}\right) \delta 4.22(\mathrm{t}, J=$ 6.6 Hz, $2 \mathrm{H}), 3.74-3.69$ (m, $1 \mathrm{H}), 3.66$ (dd, $J=11.2,2.8 \mathrm{~Hz}, 1 \mathrm{H}), 3.44$ (dd, $J=10.8,7.6 \mathrm{~Hz}, 1 \mathrm{H}), 3.01$ (s, $3 \mathrm{H}), 2.11$ (br s, $1 \mathrm{H}), 1.97$ (br s, $1 \mathrm{H}), 1.78-1.71$ (m, $2 \mathrm{H}), 1.44-1.32$ (m, $12 \mathrm{H}) .{ }^{13} \mathbf{C}$ NMR (100 MHz, $\left.\mathrm{CDCl}_{3}\right) \delta 72.6,70.5,67.2,37.7,33.4,29.7,29.6,29.4,29.2,25.7$ (two peaks). HRMS (ESI) calcd for 
$\mathrm{C}_{11} \mathrm{H}_{24} \mathrm{O}_{5} \mathrm{SNa}[\mathrm{M}+\mathrm{Na}]^{+} 291.1242$, found: 291.1245 .<smiles>CC(C)(C)[Si](OCCC(O)CO)(c1ccccc1)c1ccccc1</smiles>

\section{4-((tert-butyldiphenylsilyl)oxy)butane-1,2-diol (2o)}

Prepared according to the general procedure $2.3,0{ }^{\circ} \mathrm{C} 8 \mathrm{~h}$, purified by flash column chromatography $(\mathrm{PE} / \mathrm{EA}=5 / 1)$, white solid $(145.0 \mathrm{mg}, 84 \%)$. m.p.: 55-56 ${ }^{\circ} \mathrm{C} .{ }^{\mathbf{1}} \mathbf{H} \mathbf{~ N M R}\left(400 \mathrm{MHz}, \mathrm{CDCl}_{3}\right) \delta$ 7.70-7.68 (m, $4 \mathrm{H})$, 7.48-7.39 (m, $6 \mathrm{H}), 4.04-3.99(\mathrm{~m}, 1 \mathrm{H}), 3.91-3.85(\mathrm{~m}, 2 \mathrm{H}), 3.66(\mathrm{dd}, J=11.2,2.8 \mathrm{~Hz}, 1 \mathrm{H})$, 3.54 (dd, $J=11.4,6.6 \mathrm{~Hz}, 1 \mathrm{H}), 3.51$ (br s, $1 \mathrm{H}), 2.64$ (br s, $1 \mathrm{H}), 1.84-1.75(\mathrm{~m}, 1 \mathrm{H}), 1.69-1.62(\mathrm{~m}, 1 \mathrm{H})$, $1.08(\mathrm{~s}, 9 \mathrm{H}) .{ }^{13} \mathrm{C}$ NMR $\left(100 \mathrm{MHz}, \mathrm{CDCl}_{3}\right) \delta 135.8(\mathrm{~d}, J=1.6 \mathrm{~Hz}), 133.2(\mathrm{~d}, J=7.0 \mathrm{~Hz}), 130.2,128.1$, 71.9, 67.0, 62.8, 35.1, 27.1,19.4. HRMS (ESI) calcd for $\mathrm{C}_{20} \mathrm{H}_{28} \mathrm{O}_{3} \mathrm{SiNa}[\mathrm{M}+\mathrm{Na}]^{+} 367.1705$, found: 367.1706.

$\mathrm{OAC}_{\mathrm{OAc}}^{\mathrm{BnO}}$ acetic acid 2-acetoxy-3-benzyloxypropyl ester $(\mathbf{4 a})^{13}$

Prepared according to the general procedure $2.4,4 \mathrm{~h}$, purified by flash column chromatography (PE/EA = 20/1), colorless oil (67.6 mg, 51\%). ${ }^{1} \mathbf{H}$ NMR (400 MHz, $\left.\mathrm{CDCl}_{3}\right) \delta$ 7.37-7.27 (m, $\left.5 \mathrm{H}\right)$, 5.24-5.19 (m, 1 $\mathrm{H}), 4.64(\mathrm{~d}, J=12.0 \mathrm{~Hz}, 1 \mathrm{H}), 4.51(\mathrm{~d}, J=12.4 \mathrm{~Hz}, 1 \mathrm{H}), 4.34(\mathrm{dd}, J=12.0,4.0 \mathrm{~Hz}, 1 \mathrm{H}), 4.19(\mathrm{dd}, J=$ 11.8, 6.2 Hz, $1 \mathrm{H}), 3.59$ (d, J = 5.2 Hz, $2 \mathrm{H}), 2.08$ (s, $3 \mathrm{H}), 2.03$ (s, $3 \mathrm{H})$.<smiles>CC(=O)OCC(C)OC(C)=O</smiles>

\section{Pentane-1,2-diyl diacetate $(4 b)^{14}$}

Prepared according to the general procedure $2.4,4 \mathrm{~h}$, purified by flash column chromatography $(\mathrm{PE} / \mathrm{EA}=$ 50/1), colorless oil (76.2 mg, 75\%). ${ }^{1} \mathbf{H}$ NMR (400 MHz, $\left.\mathrm{CDCl}_{3}\right) \delta$ 5.05-4.98 (m, $\left.1 \mathrm{H}\right), 4.17(\mathrm{dd}, J=12.0$, $3.2 \mathrm{~Hz}, 1 \mathrm{H}), 3.97$ (dd, $J=11.8,6.6 \mathrm{~Hz}, 1 \mathrm{H}), 2.01$ (s, $3 \mathrm{H}), 2.00(\mathrm{~s}, 3 \mathrm{H}), 1.55-1.50(\mathrm{~m}, 2 \mathrm{H}), 1.33-1.20$ $(\mathrm{m}, 4 \mathrm{H}), 0.84(\mathrm{t}, J=6.8 \mathrm{~Hz}, 3 \mathrm{H})$.<smiles>CC(=O)OCC(C)OC(C)=O</smiles>

\section{Octane-1, 2-diyldiacetate $(4 c)^{15}$}

Prepared according to the general procedure $2.4,4 \mathrm{~h}$, purified by flash column chromatography $(\mathrm{PE} / \mathrm{EA}=$ 50/1), colorless oil (75.6 mg, 66\%). ${ }^{1} \mathbf{H}$ NMR (400 MHz, $\left.\mathrm{CDCl}_{3}\right) \delta$ 5.07-5.02 (m, $\left.1 \mathrm{H}\right), 4.20(\mathrm{dd}, J=12.0$, 
$3.2 \mathrm{~Hz}, 1 \mathrm{H}), 4.00(\mathrm{dd}, J=12.0,6.8 \mathrm{~Hz}, 1 \mathrm{H}), 2.04$ (s, two peaks, $6 \mathrm{H}), 1.58-1.50(\mathrm{~m}, 2 \mathrm{H}), 1.27-1.25(\mathrm{~m}$, $8 \mathrm{H}), 0.85(\mathrm{t}, J=6.6 \mathrm{~Hz}, 3 \mathrm{H})$.<smiles>CC(=O)OCC(C)OC(C)=O</smiles>

\section{Dodecane-1,2-diyl diacetate $(4 \mathrm{~d})^{13}$}

Prepared according to the general procedure $2.4,4 \mathrm{~h}$, purified by flash column chromatography $(\mathrm{PE} / \mathrm{EA}=$ 50/1), colorless oil $(85.5 \mathrm{mg}, 60 \%) .{ }^{1} \mathbf{H}$ NMR $\left(400 \mathrm{MHz}, \mathrm{CDCl}_{3}\right) \delta 5.07-5.01(\mathrm{~m}, 1 \mathrm{H}), 4.20(\mathrm{dd}, J=11.6$, $3.2 \mathrm{~Hz}, 1 \mathrm{H}), 4.00$ (dd, $J=11.8,6.6 \mathrm{~Hz}, 1 \mathrm{H}), 2.04$ (s, $3 \mathrm{H}), 2.03$ (s, $3 \mathrm{H}), 1.57-1.52(\mathrm{~m}, 2 \mathrm{H}), 1.27-1.23$ $(\mathrm{m}, 16 \mathrm{H}), 0.85(\mathrm{t}, J=6.4 \mathrm{~Hz}, 3 \mathrm{H})$.<smiles>CC(=O)OCC(C)OC(C)=O</smiles>

\section{6-bromohexane-1,2-diyldiacetate (4e)}

Prepared according to the general procedure $2.4,4 \mathrm{~h}$, purified by flash column chromatography (PE/EA = 20/1), colorless oil (122.5 mg, 87\%). ${ }^{1} \mathbf{H}$ NMR (400 MHz, $\left.\mathrm{CDCl}_{3}\right) \delta 5.07-5.01(\mathrm{~m}, 1 \mathrm{H}), 4.19(\mathrm{dd}, J=$ 12.0, 3.6 Hz, $1 \mathrm{H}), 4.00(\mathrm{dd}, J=12.0,6.4 \mathrm{~Hz}, 1 \mathrm{H}), 3.37$ (t, J=6.6 Hz, $2 \mathrm{H}), 2.04(\mathrm{~s}, 3 \mathrm{H}), 2.03(\mathrm{~s}, 3 \mathrm{H})$, 1.88-1.80 (m, $2 \mathrm{H}), 1.62-1.54$ (m, $2 \mathrm{H}), 1.52-1.41$ (m, $2 \mathrm{H}) .{ }^{13} \mathbf{C}$ NMR (100 MHz, $\left.\mathrm{CDCl}_{3}\right) \delta 170.8,170.6$, 71.2, 65.0, 33.4, 32.4, 29.9, 23.8, 21.1, 20.9. HRMS (ESI) calcd for $\mathrm{C}_{10} \mathrm{H}_{17} \mathrm{O}_{4} \mathrm{BrNa}[\mathrm{M}+\mathrm{Na}]^{+}$303.0208, found: 303.0204 .

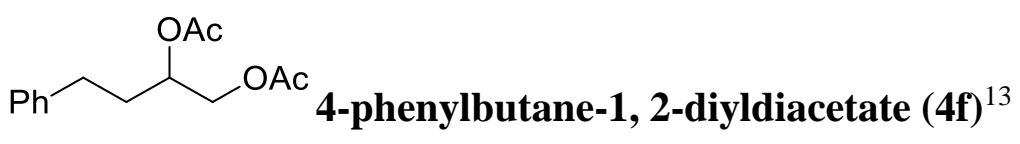

Prepared according to the general procedure $2.4,4 \mathrm{~h}$, purified by flash column chromatography (PE/EA = 20/1), colorless oil (99.6 mg, 80\%). ${ }^{1} \mathbf{H}$ NMR (400 MHz, $\left.\mathrm{CDCl}_{3}\right) \delta$ 7.30-7.25 (m, $\left.2 \mathrm{H}\right)$, 7.21-7.16 (m, 3 H), 5.14-5.08 (m, $1 \mathrm{H}), 4.24(\mathrm{dd}, J=11.8,3.4 \mathrm{~Hz}, 1 \mathrm{H}), 4.06(\mathrm{dd}, J=12.0,6.4 \mathrm{~Hz}, 1 \mathrm{H}), 2.73-2.59$ (m, 2 H), 2.06 (s, $3 \mathrm{H}), 2.05$ (s, $3 \mathrm{H}), 2.03-1.83(\mathrm{~m}, 2 \mathrm{H})$.<smiles>CC(=O)OCC(CCc1ccc(C(F)(F)F)cc1)OC(C)=O</smiles>

\section{4-(4-(trifluoromethyl)phenyl)butane-1,2-diyl diacetate (4g)}

Prepared according to the general procedure $2.4,4 \mathrm{~h}$, purified by flash column chromatography $(\mathrm{PE} / \mathrm{EA}=$ 
10/1), yellow oil (143.6 mg, 90\%). ${ }^{1} \mathbf{H}$ NMR (400 MHz, $\left.\mathrm{CDCl}_{3}\right) \delta 7.55(\mathrm{~d}, J=8.0 \mathrm{~Hz}, 2 \mathrm{H}), 7.29(\mathrm{~d}, J=$ $8.0 \mathrm{~Hz}, 2 \mathrm{H}), 5.14-5.08(\mathrm{~m}, 1 \mathrm{H}), 4.25(\mathrm{dd}, J=12.0,3.6 \mathrm{~Hz}, 1 \mathrm{H}), 4.07$ (dd, $J=12.0,6.4 \mathrm{~Hz}, 1 \mathrm{H}), 2.79$ $2.66(\mathrm{~m}, 2 \mathrm{H}), 2.07(\mathrm{~s}, 3 \mathrm{H}), 2.06(\mathrm{~s}, 3 \mathrm{H}), 2.02-1.85(\mathrm{~m}, 2 \mathrm{H}) .{ }^{13} \mathbf{C}$ NMR $\left(100 \mathrm{MHz}, \mathrm{CDCl}_{3}\right) \delta 171.0$, 170.8, 145.4, 129.0, $128.8(\mathrm{q}, J=32.4 \mathrm{~Hz}), 125.7(\mathrm{q}, J=3.5 \mathrm{~Hz}), 71.2,65.1,32.4,31.7,21.2,20.9 .{ }^{19} \mathbf{F}$ NMR $\left(376 \mathrm{MHz}, \mathrm{CDCl}_{3}\right) \delta$-62.4. HRMS (ESI) calcd for $\mathrm{C}_{15} \mathrm{H}_{18} \mathrm{~F}_{3} \mathrm{O}_{4}[\mathrm{M}+\mathrm{H}]^{+}$319.1152, found: 319.1149 .<smiles>CC(=O)OCC(C)OC(C)=O</smiles>

\section{0, 11-diacetoxyundecanoic acid $(4 h)^{13}$}

Prepared according to the general procedure 2.4, $10 \mathrm{~mL}$ of $\mathrm{AcOH}$ used as solvent, $4 \mathrm{~h}$, purified by flash column chromatography $(\mathrm{PE} / \mathrm{EA}=10 / 1)$, colorless oil $(132.3 \mathrm{mg}, 88 \%) .{ }^{1} \mathbf{H} \mathbf{N M R}\left(400 \mathrm{MHz}, \mathrm{CDCl}_{3}\right) \delta$ 5.09-5.03 (m, $1 \mathrm{H}), 4.22(\mathrm{dd}, J=11.8,3.4 \mathrm{~Hz}, 1 \mathrm{H}), 4.02(\mathrm{dd}, J=11.8,6.6 \mathrm{~Hz}, 1 \mathrm{H}), 2.34(\mathrm{t}, J=7.6 \mathrm{~Hz}$, $2 \mathrm{H}), 2.07$ (s, $3 \mathrm{H}), 2.06$ (s, $3 \mathrm{H}), 1.66-1.56$ (m, $4 \mathrm{H}), 1.29-1.25(\mathrm{~m}, 10 \mathrm{H})$.<smiles>CC(=O)OCC(C)OC(C)=O</smiles>

\section{1-oxo-11-phenylundecane-1, 2-diyldiacetate (4i)}

Prepared according to the general procedure $2.4,4 \mathrm{~h}$, purified by flash column chromatography (PE/EA = 20/1), white solid (145.3 mg, 80\%). m.p.: 39-41 ${ }^{\circ} \mathrm{C} .{ }^{1} \mathbf{H}$ NMR (400 MHz, $\left.\mathrm{CDCl}_{3}\right) \delta$ 7.97-7.95 (m, $\left.2 \mathrm{H}\right)$, 7.58-7.54 (m, $1 \mathrm{H}), 7.48-7.44(\mathrm{~m}, 2 \mathrm{H}), 5.10-5.04(\mathrm{~m}, 1 \mathrm{H}), 4.22(\mathrm{dd}, J=12.0,3.2 \mathrm{~Hz}, 1 \mathrm{H}), 4.03(\mathrm{dd}, J=$ 11.8, 6.6 Hz, $1 \mathrm{H}), 2.96$ (t, J = 7.4 Hz, $2 \mathrm{H}), 2.07$ (s, $3 \mathrm{H}), 2.06$ (s, $3 \mathrm{H}), 1.77-1.70(\mathrm{~m}, 2 \mathrm{H}), 1.61-1.52(\mathrm{~m}$, $2 \mathrm{H}), 1.41-1.25(\mathrm{~m}, 10 \mathrm{H}) .{ }^{13} \mathbf{C}$ NMR $\left(100 \mathrm{MHz}, \mathrm{CDCl}_{3}\right) \delta 200.7,171.0,170.8,137.4,133.1,128.8,128.3$, 71.8, 65.4, 38.8, 30.9, 29.6, 29.5 (two peaks), 25.3, 24.5, 21.3, 21.0. HRMS (ESI) calcd for $\mathrm{C}_{21} \mathrm{H}_{30} \mathrm{O}_{5} \mathrm{Na}$ [M+Na $]^{+}$385.1991, found: 385.1989.<smiles>CC(=O)OCC(C)OC(C)=O</smiles>

\section{1-oxododecane-1,2-diyl diacetate $(4 \mathbf{j})$}

Prepared according to the general procedure $2.4,4 \mathrm{~h}$, purified by flash column chromatography $(\mathrm{PE} / \mathrm{EA}=$ 20/1), colorless oil (117.3 mg, 78\%). ${ }^{1} \mathbf{H}$ NMR (400 MHz, $\left.\mathrm{CDCl}_{3}\right) \delta$ 5.06-5.00 (m, $\left.1 \mathrm{H}\right), 4.19$ (dd, $J=$ 11.8, 3.4 Hz, $1 \mathrm{H}), 3.99$ (dd, $J=11.8,6.6 \mathrm{~Hz}, 1 \mathrm{H}), 2.38$ (t, $J=7.4 \mathrm{~Hz}, 2 \mathrm{H}), 2.10$ (s, $3 \mathrm{H}) 2.04$ (s, $3 \mathrm{H}$ ), 2.03 (s, $3 \mathrm{H}), 1.54-1.51(\mathrm{~m}, 4 \mathrm{H}), 1.24$ (br s, $10 \mathrm{H}) .{ }^{13} \mathrm{C}$ NMR $\left(100 \mathrm{MHz}, \mathrm{CDCl}_{3}\right) \delta 209.5,171.1,170.9$, 71.8, 65.4, 44.0, 30.9, 30.1, 29.5 (three peaks), 29.3, 25.3, 24.0, 21.3, 21.0. HRMS (ESI) calcd for 
$\mathrm{C}_{16} \mathrm{H}_{28} \mathrm{O}_{5} \mathrm{Na}[\mathrm{M}+\mathrm{Na}]^{+}$323.1837, found: 323.1834.<smiles>CON(C)C(=O)CC(COC(C)=O)OC(C)=O</smiles>

\section{1-(methoxy(methyl)amino)-11-oxoundecane-1,2-diyldiacetate (4k)}

Prepared according to the general procedure $2.4,4 \mathrm{~h}$, purified by flash column chromatography $(\mathrm{PE} / \mathrm{EA}=$ 10/1), colorless oil (146.9 mg, 85\%). ${ }^{1} \mathbf{H}$ NMR (400 MHz, $\left.\mathrm{CDCl}_{3}\right) \delta 5.07-5.01(\mathrm{~m}, 1 \mathrm{H}), 4.20(\mathrm{dd}, J=$ 11.8, $3.4 \mathrm{~Hz}, 1 \mathrm{H}), 4.00(\mathrm{dd}, J=11.8,6.6 \mathrm{~Hz}, 1 \mathrm{H}), 3.66(\mathrm{~s}, 3 \mathrm{H}), 3.15(\mathrm{~s}, 3 \mathrm{H}), 2.38(\mathrm{t}, J=7.6 \mathrm{~Hz}, 2 \mathrm{H})$, 2.04 (s, two peaks, $6 \mathrm{H}), 1.61-1.53(\mathrm{~m}, 4 \mathrm{H}), 1.28$ (s, $10 \mathrm{H}) .{ }^{13} \mathbf{C ~ N M R}\left(100 \mathrm{MHz}, \mathrm{CDCl}_{3}\right) \delta 175.0,171.0$, 170.8, 71.8, 65.3, 61.4, 32.4, 32.1, 30.9, 29.6, 29.5 (two peaks), 29.4, 25.3, 24.8, 21.3, 21.0. HRMS (ESI) calcd for $\mathrm{C}_{17} \mathrm{H}_{31} \mathrm{NO}_{6} \mathrm{Na}[\mathrm{M}+\mathrm{Na}]^{+}$368.2049, found: 368.2046.<smiles>CC(=O)OCC(C)OC(C)=O</smiles>

\section{4-(1,3-dioxoisoindolin-2-yl)butane-1,2-diyldiacetate (4I)}

Prepared according to the general procedure $2.4,4 \mathrm{~h}$, purified by flash column chromatography $(\mathrm{PE} / \mathrm{EA}=$ 10/1), colorless oil (149.1 mg, 93\%). ${ }^{1} \mathbf{H}$ NMR (400 MHz, $\left.\mathrm{CDCl}_{3}\right) \delta$ 7.87-7.83 (m, $\left.2 \mathrm{H}\right), 7.74-7.70$ (m, 2 H), 5.08-5.03 (m, $1 \mathrm{H}), 4.28(\mathrm{dd}, J=12.0,4.0 \mathrm{~Hz}, 1 \mathrm{H}), 4.09(\mathrm{dd}, J=11.6,5.6 \mathrm{~Hz}, 1 \mathrm{H}), 3.86-3.79(\mathrm{~m}, 1$ H), 3.77-3.70 (m, $1 \mathrm{H}), 2.09$ (s, two peaks, $6 \mathrm{H}), 2.07-1.99(\mathrm{~m}, 2 \mathrm{H}) .{ }^{13} \mathbf{C} \mathbf{N M R}\left(100 \mathrm{MHz}, \mathrm{CDCl}_{3}\right) \delta 170.9$, 170.7, 168.4, 134.3, 132.3, 123.6, 69.2, 64.8, 34.4, 29.6, 21.2, 21.0. HRMS (ESI) calcd for $\mathrm{C}_{16} \mathrm{H}_{17} \mathrm{NO}_{6} \mathrm{Na}$ $[\mathrm{M}+\mathrm{Na}]^{+}$342.0954, found: 342.0956 .<smiles>CC(=O)OCC(C)OCC(C)O</smiles>

\section{0-(tosyloxy)decane-1,2-diyl diacetate (4m)}

Prepared according to the general procedure $2.4,4 \mathrm{~h}$, purified by flash column chromatography (PE/EA = 20/1), colorless oil (162.7 mg, 76\%). ${ }^{1} \mathbf{H}$ NMR (400 MHz, $\left.\mathrm{CDCl}_{3}\right) \delta 7.76(\mathrm{~d}, J=8.4 \mathrm{~Hz}, 2 \mathrm{H}), 7.33(\mathrm{~d}, J$ $=8.0 \mathrm{~Hz}, 2 \mathrm{H}), 5.06-5.00(\mathrm{~m}, 1 \mathrm{H}), 4.20(\mathrm{dd}, J=12.0,3.2 \mathrm{~Hz}, 1 \mathrm{H}), 4.02-3.97(\mathrm{~m}, 3 \mathrm{H}), 2.43(\mathrm{~s}, 3 \mathrm{H})$, 2.04 (s, two peaks, $6 \mathrm{H}), 1.64-1.57$ (m, $2 \mathrm{H}), 1.55-1.50$ (m, $2 \mathrm{H}), 1.30-1.21(\mathrm{~m}, 10 \mathrm{H}) .{ }^{13} \mathbf{C}$ NMR $(100$ $\left.\mathrm{MHz}, \mathrm{CDCl}_{3}\right) \delta 171.1,171.0,145.0,133.7,130.1,128.2,71.9,71.0,65.4,31.0,29.5$ (two peaks), 29.1 (two peaks), 25.6, 25.4, 22.0, 21.4, 21.1. HRMS (ESI) calcd for $\mathrm{C}_{21} \mathrm{H}_{32} \mathrm{O}_{7} \mathrm{SNa}[\mathrm{M}+\mathrm{Na}]^{+} 451.1766$, found: 451.1765 . 
MsO

10-((methylsulfonyl)oxy)decane-1,2-diyl diacetate (4n)

Prepared according to the general procedure $2.4,4 \mathrm{~h}$, purified by flash column chromatography $(\mathrm{PE} / \mathrm{EA}=$ 5/1), colorless oil (156.6 mg, 89\%). ${ }^{1} \mathbf{H}$ NMR (400 MHz, $\left.\mathrm{CDCl}_{3}\right) \delta$ 5.07-5.01 (m, $\left.1 \mathrm{H}\right), 4.22-4.18$ (m, 3 H), $4.00(\mathrm{dd}, J=11.8,6.6 \mathrm{~Hz}, 1 \mathrm{H}), 2.98$ (s, $3 \mathrm{H}), 2.05$ (s, $3 \mathrm{H}), 2.04$ (s, $3 \mathrm{H}), 1.75-1.68$ (m, 2 H), 1.591.50 (m, $2 \mathrm{H}), 1.39-1.28$ (m, $10 \mathrm{H}) .{ }^{13} \mathbf{C} \mathbf{N M R}\left(100 \mathrm{MHz}, \mathrm{CDCl}_{3}\right) \delta 171.1,170.9,71.8,70.4,65.4,37.6$, 30.9, 29.4, 29.3, 29.1, 25.6, 25.3, 21.4, 21.1. HRMS (ESI) calcd for $\mathrm{C}_{15} \mathrm{H}_{28} \mathrm{O}_{7} \mathrm{SNa}[\mathrm{M}+\mathrm{Na}]^{+} 375.1453$, found: 375.1456 .<smiles>CC(=O)OCC(CCO[Si](C(C)=O)(c1ccccc1)c1ccc(C)c(C)c1)OCC(C)OC(C)=O</smiles>

\section{4-((tert-butyldiphenylsilyl)oxy)butane-1,2-diyldiacetate (4o)}

Prepared according to the general procedure $2.4,4 \mathrm{~h}$, purified by flash column chromatography (PE/EA = 20/1), colorless oil (171.1 mg, 80\%). ${ }^{1} \mathbf{H}$ NMR (400 MHz, $\left.\mathrm{CDCl}_{3}\right) \delta$ 7.70-7.62 (m, $\left.4 \mathrm{H}\right)$, 7.45-7.38 (m, 6 H), 5.34-5.28 (m, $1 \mathrm{H}), 4.33(\mathrm{dd}, J=12.0,3.2 \mathrm{~Hz}, 1 \mathrm{H}), 4.10(\mathrm{dd}, J=11.8,6.2 \mathrm{~Hz}, 1 \mathrm{H}), 3.70(\mathrm{t}, J=6.4$ Hz, 2 H), 2.06 (s, $3 \mathrm{H}), 2.01$ (s, $3 \mathrm{H}), 1.88-1.83$ (m, $2 \mathrm{H}), 1.06$ (s, $9 \mathrm{H}) .{ }^{13} \mathbf{C ~ N M R}\left(100 \mathrm{MHz}, \mathrm{CDCl}_{3}\right) \delta$ $171.1,170.7,135.9,133.7,130.0,128.0,69.5,65.6,60.0,33.9,27.1,21.3,21.1,19.4$. HRMS (ESI) calcd for $\mathrm{C}_{24} \mathrm{H}_{32} \mathrm{O} 5 \mathrm{SiNa}[\mathrm{M}+\mathrm{Na}]^{+} 451.1917$, found: 451.1920 .

\subsection{General procedure for difluorination of alkenes}

$\mathrm{Pd}\left(\mathrm{CH}_{3} \mathrm{CN}\right)_{2} \mathrm{Cl}_{2}(7.5 \mathrm{~mol} \%, 9.7 \mathrm{mg})$ was weighed directly into a $50 \mathrm{~mL}$ round-bottomed flask, charged with a stir bar and dried under high vacuum for 15 mins, purge oxygen or air 3 times. Under an atmosphere of oxygen or air (1 atm, balloon) $5 \mathrm{~mL}$ of $\mathrm{AcOH}$ and ${ }^{t} \mathrm{BuONO}(20 \mathrm{~mol} \%, 10.3 \mathrm{mg})$ were added and stirred at the oil bath and the indoor temperature at $25^{\circ} \mathrm{C}$. Alkene $(0.5 \mathrm{mmol})$ was then added and the reaction vessel was shielded with light using aluminum foil, the resulting reaction mixture was monitored by TLC. After completion, $\mathrm{AcOH}$ was removed under reduced pressure, then $\mathrm{MeOH}(5 \mathrm{~mL})$ and $\mathrm{K}_{2} \mathrm{CO}_{3}(2.0 \mathrm{mmol})$ were added to the flask. The reaction was then allowed to stir at $25^{\circ} \mathrm{C}$ for $8 \mathrm{~h}$. The solvent was removed under reduced pressure and the crude mixture was purified by celite using ethyl acetate as eluent, then removed the solvent to afford the 1, 2-diol compound. 1, 2-diol compound was dissolved in $\operatorname{dry} \mathrm{CH}_{2} \mathrm{Cl}_{2}$ $(5.0 \mathrm{~mL})$ at $0{ }^{\circ} \mathrm{C}$, diethylaminosulfurtrifluoride $(0.48 \mathrm{~g}, 3 \mathrm{mmol})$ was added and the reaction was allowed 
to warm to room temperature for $24 \mathrm{~h}$. The reaction mixture was diluted with $\mathrm{CH}_{2} \mathrm{Cl}_{2}(20 \mathrm{~mL})$, washed with saturated $\mathrm{NaHCO}_{3}(10 \mathrm{~mL})$ and $\mathrm{NaCl}(10 \mathrm{~mL})$, the organic phase was dried with $\mathrm{Na}_{2} \mathrm{SO}_{4}$, filtered and concentrated in vacuo. The crude product was purified by column chromatography on silica gel using petroleum ether and ethyl acetate as eluent to afford the corresponding products.

\subsection{General procedure for dichlorination of alkenes}

$\mathrm{Pd}\left(\mathrm{CH}_{3} \mathrm{CN}\right)_{2} \mathrm{Cl}_{2}(7.5 \mathrm{~mol} \%, 9.7 \mathrm{mg})$ was weighed directly into a $50 \mathrm{~mL}$ round-bottomed flask, charged with a stir bar and dried under high vacuum for 15 mins, purge oxygen or air 3 times. Under an atmosphere of oxygen or air (1 atm, balloon) $5 \mathrm{~mL}$ of $\mathrm{AcOH}$ and ${ }^{t} \mathrm{BuONO}(20 \mathrm{~mol} \%, 10.3 \mathrm{mg})$ were added and stirred at the oil bath and the indoor temperature at $25^{\circ} \mathrm{C}$. Alkene $(0.5 \mathrm{mmol})$ was then added and the reaction vessel was shielded with light using aluminum foil, the resulting reaction mixture was monitored by TLC. After completion, $\mathrm{AcOH}$ was removed under reduced pressure, then $\mathrm{MeOH}(5 \mathrm{~mL})$ and $\mathrm{K}_{2} \mathrm{CO}_{3}(2.0 \mathrm{mmol})$ were added to the flask. The reaction was then allowed to stir at $25^{\circ} \mathrm{C}$ for $8 \mathrm{~h}$. Then the solvent was removed under reduced pressure and the crude mixture was purified by celite using ethyl acetate as eluent, then removed the solvent to afford the 1, 2-diol compound. 1, 2-diol compound dissolved in $\mathrm{CH}_{2} \mathrm{Cl}_{2}(2.5$ $\mathrm{mL})$ and pyridine $(0.71 \mathrm{~g}, 9 \mathrm{mmol})$ then the reaction mixture was cooled to $-78^{\circ} \mathrm{C}, \mathrm{SO}_{2} \mathrm{Cl}_{2}(0.41 \mathrm{~g}, 3 \mathrm{mmol})$ was added and the reaction was allowed to warm to room temperature. After 8 hours the reaction mixture was diluted with $\mathrm{CH}_{2} \mathrm{Cl}_{2}(20 \mathrm{~mL})$, washed with $1 \mathrm{M} \mathrm{HCl}(5 \mathrm{~mL})$ and saturated $\mathrm{NaHCO}_{3}(10 \mathrm{~mL})$, the organic phase was dried with $\mathrm{Na}_{2} \mathrm{SO}_{4}$, filtered and concentrated in vacuo. The crude product was purified by column chromatography on silica gel using petroleum ether and ethyl acetate as eluent to afford the corresponding products.<smiles>O=C([Al]c1ccccc1)C(F)CF</smiles>

\section{0,11-difluoro-1-phenylundecan-1-one (5a)}

Prepared according to the general procedure 2.5, purified by flash column chromatography $(\mathrm{PE} / \mathrm{EA}=$ 100/1), white solid (0.5 mmol scale: $94.2 \mathrm{mg}, 67 \%$; gram-scale: 1.28 g, $57 \%)$. m.p.: $49{ }^{\circ} \mathrm{C} .{ }^{1} \mathbf{H}$ NMR $(400$ $\left.\mathrm{MHz} \mathrm{CDCl}_{3}\right) \delta$ 7.97-7.95 (m, $\left.2 \mathrm{H}\right), 7.56(\mathrm{t}, J=7.2 \mathrm{~Hz}, 1 \mathrm{H}), 7.46(\mathrm{t}, J=7.2 \mathrm{~Hz}, 2 \mathrm{H}), 4.78-4.60(\mathrm{~m}, 1 \mathrm{H})$, 4.59-4.34 (m, 2 H), 2.96 (t, J = 7.4 Hz, 2 H), 1.77-1.45 (m, $6 \mathrm{H}), 1.40-1.33$ (m, 8 H). ${ }^{13}$ C NMR (100 MHz, $\left.\mathrm{CDCl}_{3}\right) \delta 200.9,137.5,133.2,128.9,128.4,92.2(\mathrm{dd}, J=171.4,19.6 \mathrm{~Hz}), 84.5(\mathrm{dd}, J=172.4,23.1 \mathrm{~Hz})$, 38.9, 30.3 (dd, $J=20.3,6.9 \mathrm{~Hz}), 29.6$ (three peaks), 29.5, 25.1 (d, $J=4.2 \mathrm{~Hz}), 24.6 .{ }^{19} \mathbf{F ~ N M R}(376 \mathrm{MHz}$, $\left.\mathrm{CDCl}_{3}\right)-188.82(\mathrm{~d}, J=13.5 \mathrm{~Hz}),-229.8(\mathrm{~d}, J=13.5 \mathrm{~Hz})$. HRMS (ESI) calcd for calcd for $\mathrm{C}_{17} \mathrm{H}_{25} \mathrm{~F}_{2} \mathrm{O}$ 
$[\mathrm{M}+\mathrm{H}]^{+} 283.1868$, found 283.1868 .<smiles>CON(C)C(=O)[18CH]CF</smiles>

\section{0,11-difluoro- $N$-methoxy- $N$-methylundecanamide (5b)}

Prepared according to the general procedure 2.5, purified by flash column chromatography $(\mathrm{PE} / \mathrm{EA}=$ 10/1), yellow oil (80.6 mg, 61\%). ${ }^{1} \mathbf{H}$ NMR (400 MHz, $\left.\mathrm{CDCl}_{3}\right) \delta 4.78-4.60$ (m, $\left.1 \mathrm{H}\right), 4.58-4.33$ (m, $\left.2 \mathrm{H}\right)$, $3.68(\mathrm{~s}, 3 \mathrm{H}), 3.18(\mathrm{~s}, 3 \mathrm{H}), 2.41(\mathrm{t}, J=7.4 \mathrm{~Hz}, 2 \mathrm{H}), 1.76-1.31(\mathrm{~m}, 14 \mathrm{H}) .{ }^{19} \mathbf{F} \mathbf{N M R}\left(376 \mathrm{MHz}, \mathrm{CDCl}_{3}\right) \delta$ $-188.83(\mathrm{~d}, J=15.0),-229.79(\mathrm{~d}, J=15.0) .{ }^{13} \mathrm{C}$ NMR $\left(100 \mathrm{MHz}, \mathrm{CDCl}_{3}\right) \delta 175.1,92.2(\mathrm{dd}, J=171.3$, $18.9 \mathrm{~Hz}), 84.5$ (dd, $J=172.6,22.6 \mathrm{~Hz}), 61.5,32.5,32.2,30.3$ (dd, $J=20.6,6.3 \mathrm{~Hz}), 29.7,29.6,29.5,25.1$ (d, $J=4.7 \mathrm{~Hz}$ ), 24.9. HRMS (ESI) calcd for calcd for $\mathrm{C}_{13} \mathrm{H}_{26} \mathrm{NO}_{2} \mathrm{~F}_{2}[\mathrm{M}+\mathrm{H}]^{+}$266.1926, found: 266.1921 .<smiles>O=C(N=C(Cl)C(Cl)CCl)c1ccccc1</smiles>

\section{0,11-dichloro-1-phenylundecan-1-one (6a)}

Prepared according to the general procedure 2.6, purified by flash column chromatography $(\mathrm{PE} / \mathrm{EA}=$ 200/1), yellow oil (92.0 mg, 58\%). ${ }^{1} \mathbf{H}$ NMR (400 MHz, $\left.\mathrm{CDCl}_{3}\right) \delta$ 7.97-7.95 (m, $\left.2 \mathrm{H}\right), 7.56(\mathrm{t}, J=7.4 \mathrm{~Hz}$, $1 \mathrm{H}), 7.46(\mathrm{t}, J=7.0 \mathrm{~Hz}, 2 \mathrm{H}), 4.07-4.00(\mathrm{~m}, 1 \mathrm{H}), 3.76(\mathrm{dd}, J=11.4,5.0 \mathrm{~Hz}, 1 \mathrm{H}), 3.65(\mathrm{dd}, J=11.2,7.6$ $\mathrm{Hz}, 1 \mathrm{H}), 2.97(\mathrm{t}, J=7.2 \mathrm{~Hz}, 2 \mathrm{H}), 2.03-1.94(\mathrm{~m}, 1 \mathrm{H}), 1.77-1.66(\mathrm{~m}, 3 \mathrm{H}), 1.45-1.33(\mathrm{~m}, 10 \mathrm{H}) .{ }^{13} \mathbf{C} \mathbf{N M R}$ $\left(100 \mathrm{MHz}, \mathrm{CDCl}_{3}\right) \delta 200.9,137.4,133.2,128.9,128.4,61.6,48.6,38.9,35.3,29.7,29.6,29.5,29.2,26.1$, 24.6. HRMS (ESI) calcd for $\mathrm{C}_{17} \mathrm{H}_{25} \mathrm{Cl}_{2} \mathrm{O}[\mathrm{M}+\mathrm{H}]^{+} 315.1277$, found 315.1272.<smiles>CON(C)C(=O)[Hg]C(Cl)CCl</smiles>

\section{0,11-dichloro- $N$-methoxy- $N$-methylundecanamide (6b)}

Prepared according to the general procedure 2.6, purified by flash column chromatography $(\mathrm{PE} / \mathrm{EA}=$ 20/1), slightly yellow solid (93.4 mg, 63\%). m.p.: $36^{\circ} \mathrm{C}^{1}{ }^{1} \mathbf{H}$ NMR (400 MHz, $\left.\mathrm{CDCl}_{3}\right) \delta$ 4.05-3.99 (m, 1 H), $3.75(\mathrm{dd}, J=11.2,5.2 \mathrm{~Hz}, 1 \mathrm{H}), 3.67(\mathrm{~s}, 3 \mathrm{H}), 3.66-3.62(\mathrm{~m}, 3 \mathrm{H}), 3.17(\mathrm{~s}, 3 \mathrm{H}), 2.40(\mathrm{t}, J=7.6,2 \mathrm{H})$, 2.01-1.92 (m, $1 \mathrm{H}), 1.74-1.63(\mathrm{~m}, 1 \mathrm{H}), 1.60-1.31(\mathrm{~m}, 12 \mathrm{H}) .{ }^{13} \mathbf{C} \mathbf{N M R}\left(100 \mathrm{MHz}, \mathrm{CDCl}_{3}\right) \delta 175.1,61.6$, 61.5, 48.6, 35.3, 32.4, 32.1, 29.7, 29.6, 29.5, 29.2, 26.1, 24.9. HRMS (ESI) calcd for $\mathrm{C}_{13} \mathrm{H}_{26} \mathrm{NO}_{2} \mathrm{Cl}_{2}$ $[\mathrm{M}+\mathrm{H}]^{+}$298.1335, found: 298.1331 . 


\section{Kinetic Study}

\subsection{The kinetic study on the dependence of the initial rate on $\mathrm{Pd}\left(\mathrm{CH}_{3} \mathrm{CN}\right)_{2} \mathrm{Cl}_{2}$.}

General procedure: Allyl benzyl ether $(1 \mathrm{mmol}, 148 \mathrm{mg})$ was weighed directly into a $50 \mathrm{~mL}$ roundbottomed flask under the air (1 atm, balloon) condition, AcOH $(10 \mathrm{~mL})$ and ethyl 3-phenylpropionate (0.25 mmol, $45 \mathrm{mg}$ ) (as internal standard) were added, fallowed with $\boldsymbol{P d}\left(\mathrm{CH}_{3} \mathrm{CN}\right)_{2} \mathrm{Cl}_{2}(\mathbf{2 . 5}-\mathbf{1 0 . 0} \mathbf{~ m o l} \%$, 6.5-25.9 $\mathbf{~ m g}$ ) and ${ }^{t} \mathrm{BuONO}(20 \mathrm{~mol} \%, 20.6 \mathrm{mg})$ were added at the same time. The reaction flask was shielded with light using aluminum foil, the resulting reaction mixture was stirred with the oil bath and the indoor temperature at $25{ }^{\circ} \mathrm{C}$. At each sampling time $50 \mu \mathrm{L}$ reaction mixture was extracted and examined by GC. [allyl benzyl ether] was determined using ethyl 3-phenylpropionate as internal standard and the results were demonstrated in Table S2.

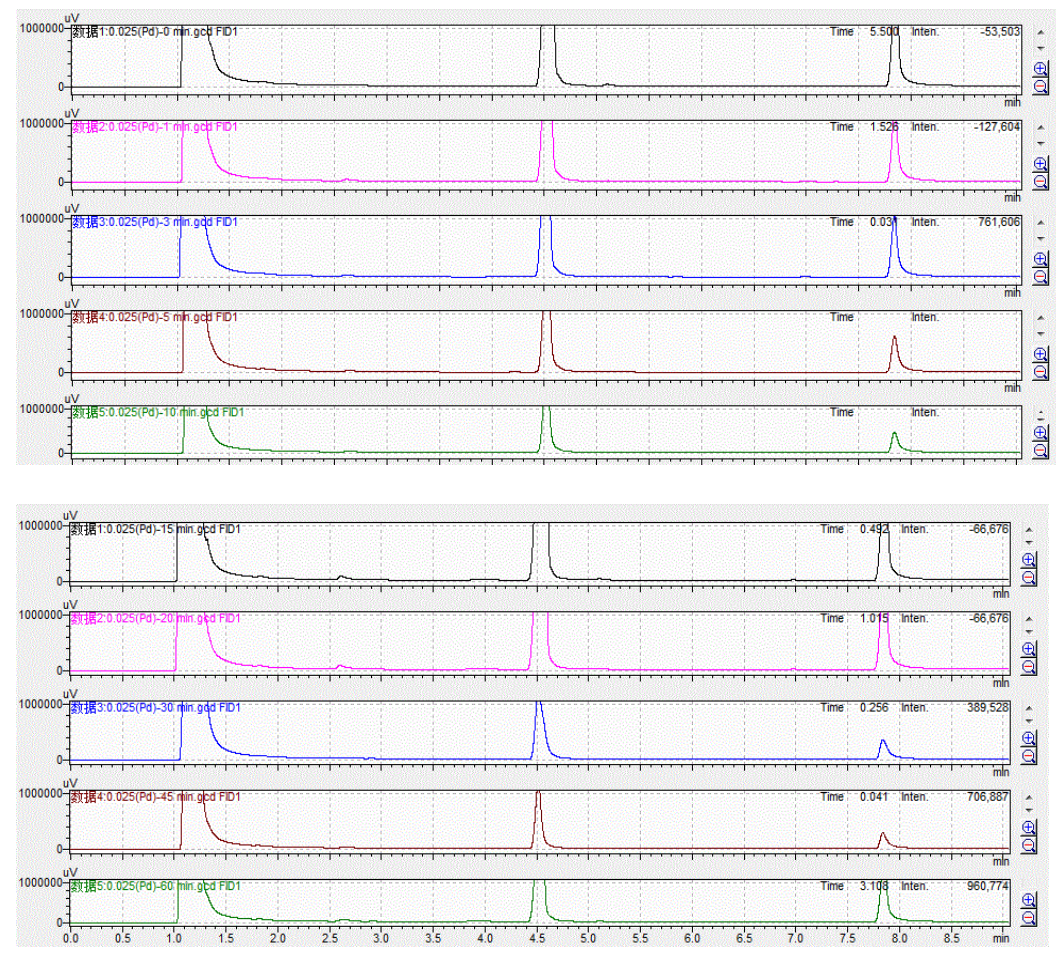

Figure S1. Conditions: [allyl benzyl ether] $=0.1 \mathrm{M}(1.0 \mathrm{mmol}, 148 \mathrm{mg})$, air (1 atm, balloon $),{ }^{t} \mathrm{BuONO}$ (20 mol \%, $20.6 \mathrm{mg}), \boldsymbol{P d}\left(\mathrm{CH}_{3} \mathrm{CN}\right)_{2} \mathrm{Cl}_{2}(2.5 \mathrm{~mol} \%$, $6.5 \mathrm{mg})$, AcOH (10 mL), $25{ }^{\circ} \mathrm{C}$, ethyl 3phenylpropionate $(0.25 \mathrm{mmol}, 45 \mathrm{mg})$ as internal standard. 


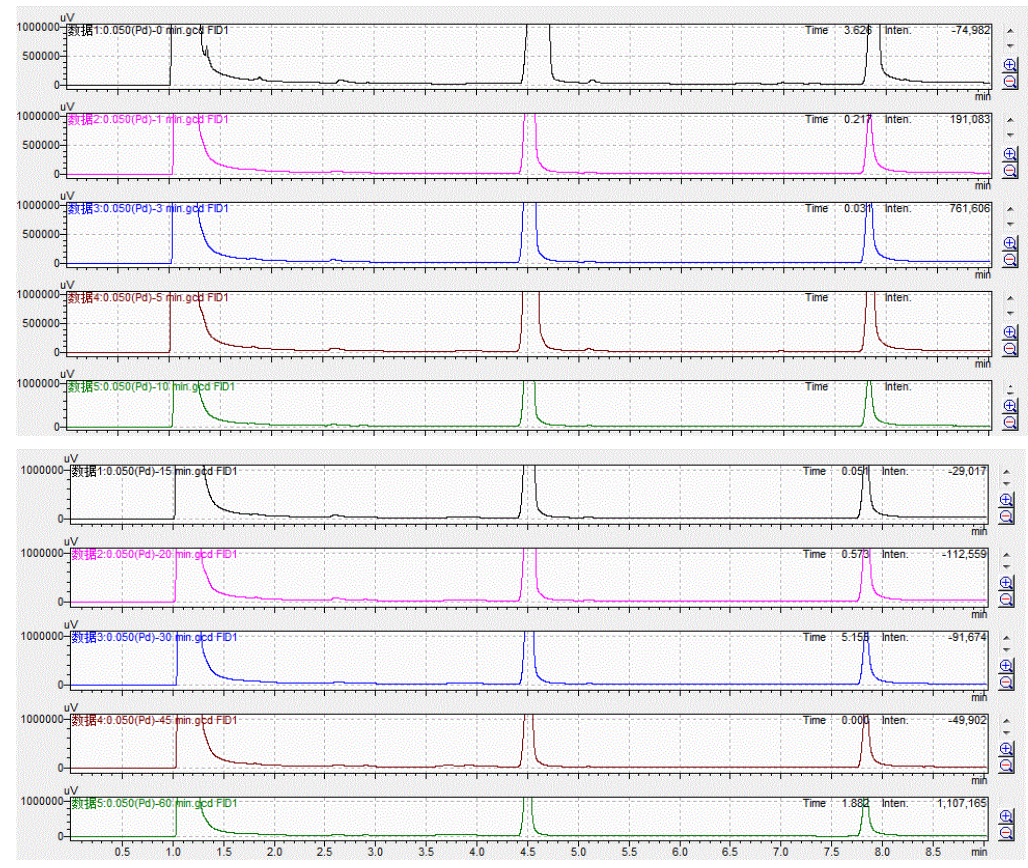

Figure S2. Conditions: [allyl benzyl ether] $=0.1 \mathrm{M}(1.0 \mathrm{mmol}, 148 \mathrm{mg})$, air $(1 \mathrm{~atm}$, balloon $),{ }^{t} \mathrm{BuONO}$ $(20 \mathrm{~mol} \%, 20.6 \mathrm{mg}), \boldsymbol{P d}\left(\mathrm{CH}_{3} \mathrm{CN}\right)_{2} \mathrm{Cl}_{2}(5.0 \mathrm{~mol} \%, 13.0 \mathrm{mg}), \mathrm{AcOH}(10 \mathrm{~mL}), 25{ }^{\circ} \mathrm{C}$, ethyl 3phenylpropionate $(0.25 \mathrm{mmol}, 45 \mathrm{mg})$ as internal standard.
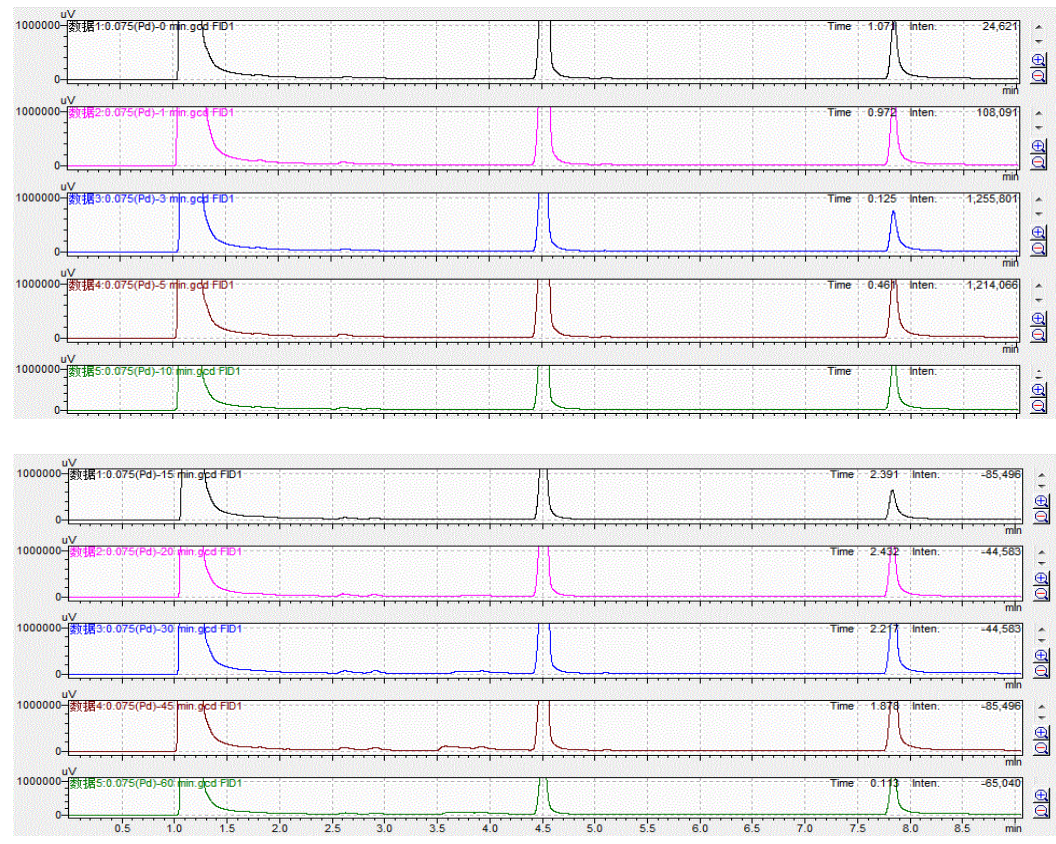

Figure S3. Conditions: [allyl benzyl ether] $=0.1 \mathrm{M}(1.0 \mathrm{mmol}, 148 \mathrm{mg})$, air (1 atm, balloon), ${ }^{t} \mathrm{BuONO}$ (20 mol \%, $20.6 \mathrm{mg}), \boldsymbol{P d}\left(\mathrm{CH}_{3} \mathrm{CN}_{2}\right)_{2} \mathrm{Cl}_{2}(7.5 \mathrm{~mol} \%, 19.5 \mathrm{mg}), \mathrm{AcOH}(10 \mathrm{~mL}), 25{ }^{\circ} \mathrm{C}$, ethyl 3phenylpropionate $(0.25 \mathrm{mmol}, 45 \mathrm{mg})$ as internal standard. 


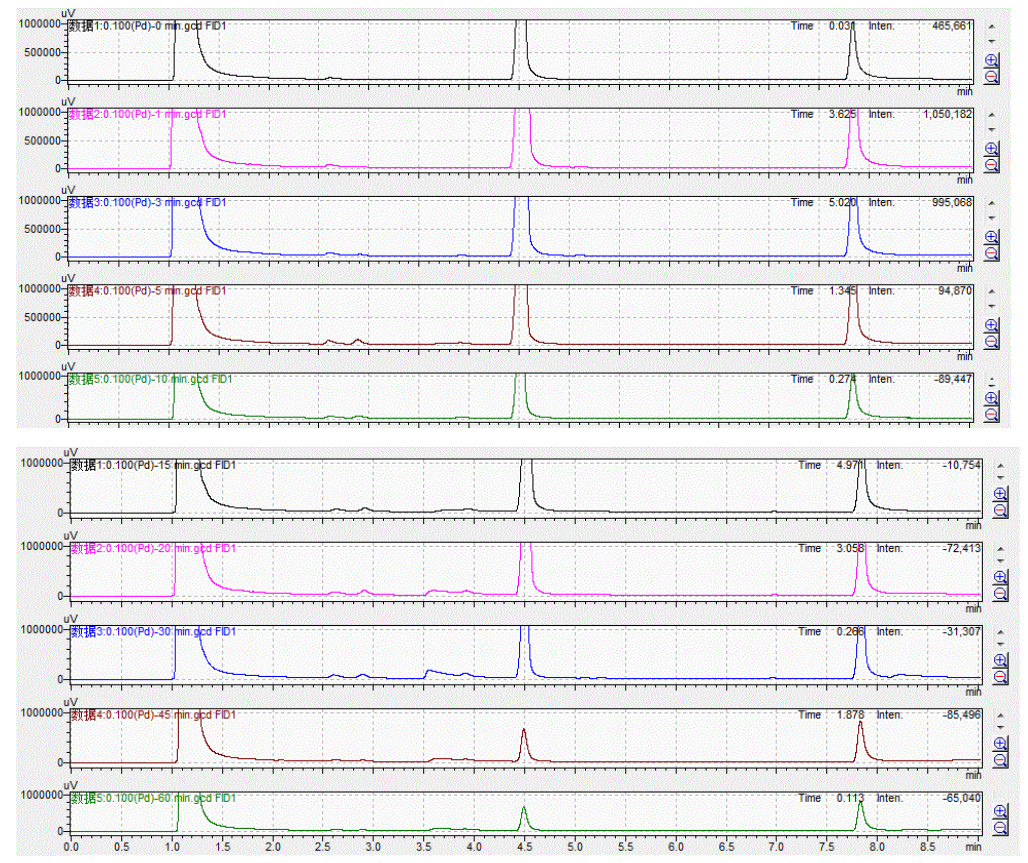

Figure S4. Conditions: [allyl benzyl ether] $=0.1 \mathrm{M}(1.0 \mathrm{mmol}, 148 \mathrm{mg})$, air $(1 \mathrm{~atm}$, balloon $),{ }^{t} \mathrm{BuONO}$ (20 mol \%, $20.6 \mathrm{mg}), \boldsymbol{P d}\left(\mathrm{CH}_{3} \mathrm{CN}\right)_{2} \boldsymbol{C l}_{2}$ (10 mol \%, $\left.25.9 \mathrm{mg}\right)$, AcOH (10 mL), $25{ }^{\circ} \mathrm{C}$, ethyl 3phenylpropionate $(0.25 \mathrm{mmol}, 45 \mathrm{mg})$ as internal standard.

Table S2. Dependence of $\mathrm{Pd}\left(\mathrm{CH}_{3} \mathrm{CN}\right)_{2} \mathrm{Cl}_{2}$ (mol \%): [allyl benzyl ether] v.s. Time. ${ }^{a}$

\begin{tabular}{|c|c|c|c|c|}
\hline \multirow{3}{*}{ Time (min) } & \multicolumn{4}{|c|}{ allyl benzyl ether (M) } \\
\hline & $\mathrm{S} 1$ & $\mathrm{~S} 2$ & $\mathrm{~S} 3$ & $\mathrm{~S} 4$ \\
\hline & $2.5 \mathrm{~mol} \mathrm{\%}$ & $5.0 \mathrm{~mol} \%$ & $7.5 \mathrm{~mol} \%$ & $10.0 \mathrm{~mol} \mathrm{\%}$ \\
\hline 0 & 0.1 & 0.1 & 0.1 & 0.1 \\
\hline 1 & 0.09853 & 0.09788 & 0.09624 & 0.09553 \\
\hline 3 & 0.09633 & 0.09466 & 0.09234 & 0.09026 \\
\hline 5 & 0.09401 & 0.09124 & 0.08788 & 0.08624 \\
\hline 10 & 0.09055 & 0.08540 & 0.07905 & 0.07442 \\
\hline 15 & 0.08702 & 0.08024 & 0.07383 & 0.06488 \\
\hline 20 & 0.08467 & 0.07409 & 0.06565 & 0.05631 \\
\hline 30 & 0.08106 & 0.06625 & 0.05893 & 0.04953 \\
\hline 45 & 0.07452 & 0.05505 & 0.04356 & 0.03477 \\
\hline 60 & 0.06723 & 0.04459 & 0.03204 & 0.02127 \\
\hline
\end{tabular}


${ }^{a}$ Conditions: [allyl benzyl ether] = 0.1 M (1.0 mmol, $\left.148 \mathrm{mg}\right)$, air (1 atm, balloon), ${ }^{t} \mathrm{BuONO}(20 \mathrm{~mol} \%$, $20.6 \mathrm{mg}), \boldsymbol{P d}\left(\mathrm{CH}_{3} \mathrm{CN}\right)_{2} \mathrm{Cl}_{2}(2.5-10.0 \mathrm{~mol} \%$, 6.5-25.9 $\mathrm{mg}), \mathrm{AcOH}(10 \mathrm{~mL}), 25{ }^{\circ} \mathrm{C}$, shielded with light, ethyl 3-phenylpropionate $(0.25 \mathrm{mmol}, 45 \mathrm{mg})$ as internal standard.

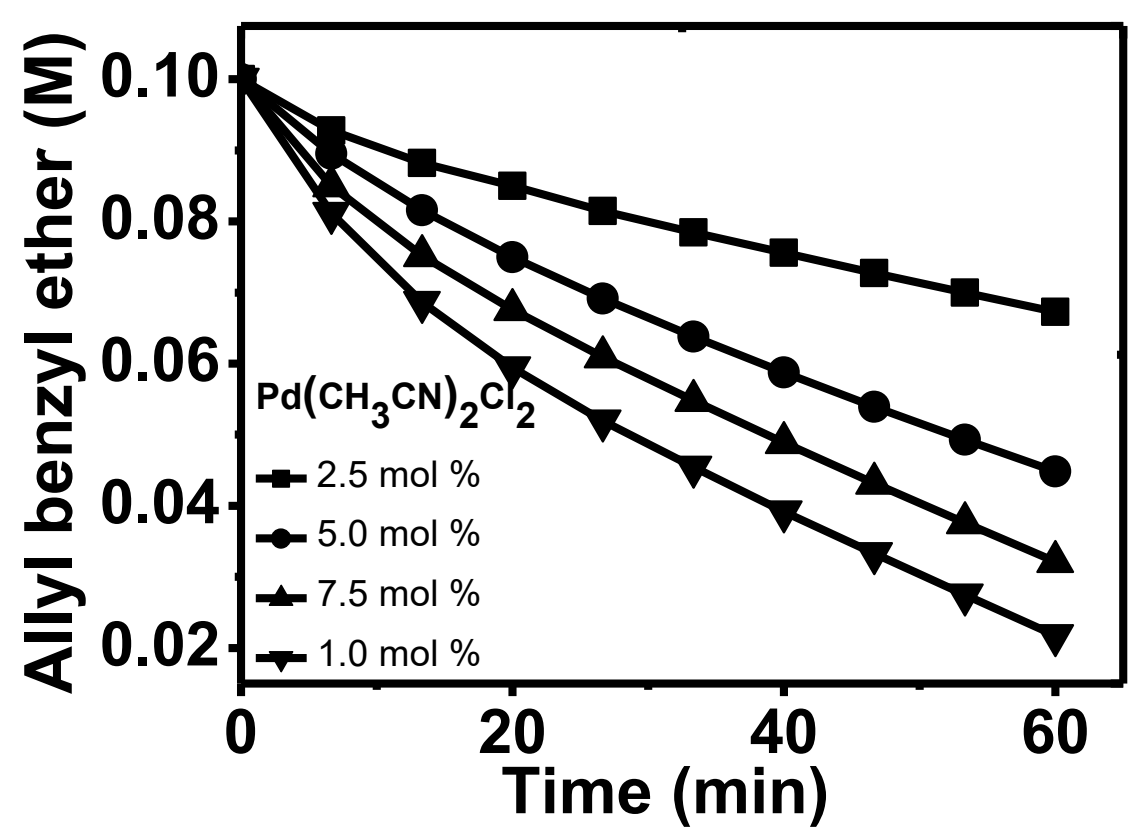

Figure 1. Dependence of the initial rate on $\mathbf{P d}\left(\mathrm{CH}_{3} \mathbf{C N}\right)_{2} \mathbf{C l}_{2}$ : Plots of [allyl benzyl ether] v.s. Time using $\operatorname{Pd}\left(\mathrm{CH}_{3} \mathrm{CN}\right)_{2} \mathrm{Cl}_{2}$.

\subsection{The dependence of the initial rate on allyl benzyl ether.}

General procedure: Allyl benzyl ether $(0.95-1.10 \mathrm{mmol}, 140.8-163.0 \mathrm{mg})$ was weighed directly into a $50 \mathrm{~mL}$ round-bottomed flask under the atmosphere of air (1 atm, balloon) condition, $\mathrm{AcOH}(10 \mathrm{~mL})$ and ethyl 3-phenylpropionate ( $0.25 \mathrm{mmol}, 45 \mathrm{mg}$ ) (as internal standard) were added, fallowed with $\operatorname{Pd}\left(\mathrm{CH}_{3} \mathrm{CN}\right)_{2} \mathrm{Cl}_{2}(7.5 \mathrm{~mol} \%, 19.5 \mathrm{mg})$ and ${ }^{t} \mathrm{BuONO}(20 \mathrm{~mol} \%, 20.6 \mathrm{mg})$ were added at the same time. The reaction flask was shielded with light using aluminum foil, the resulting reaction mixture was stirred with the oil bath and the indoor temperature at $25^{\circ} \mathrm{C}$. At each sampling time $50 \mu \mathrm{L}$ reaction mixture was extracted and examined by GC. [allyl benzyl ether] was determined using ethyl 3-phenylpropionate as internal standard and the results were demonstrated in Table S3. 


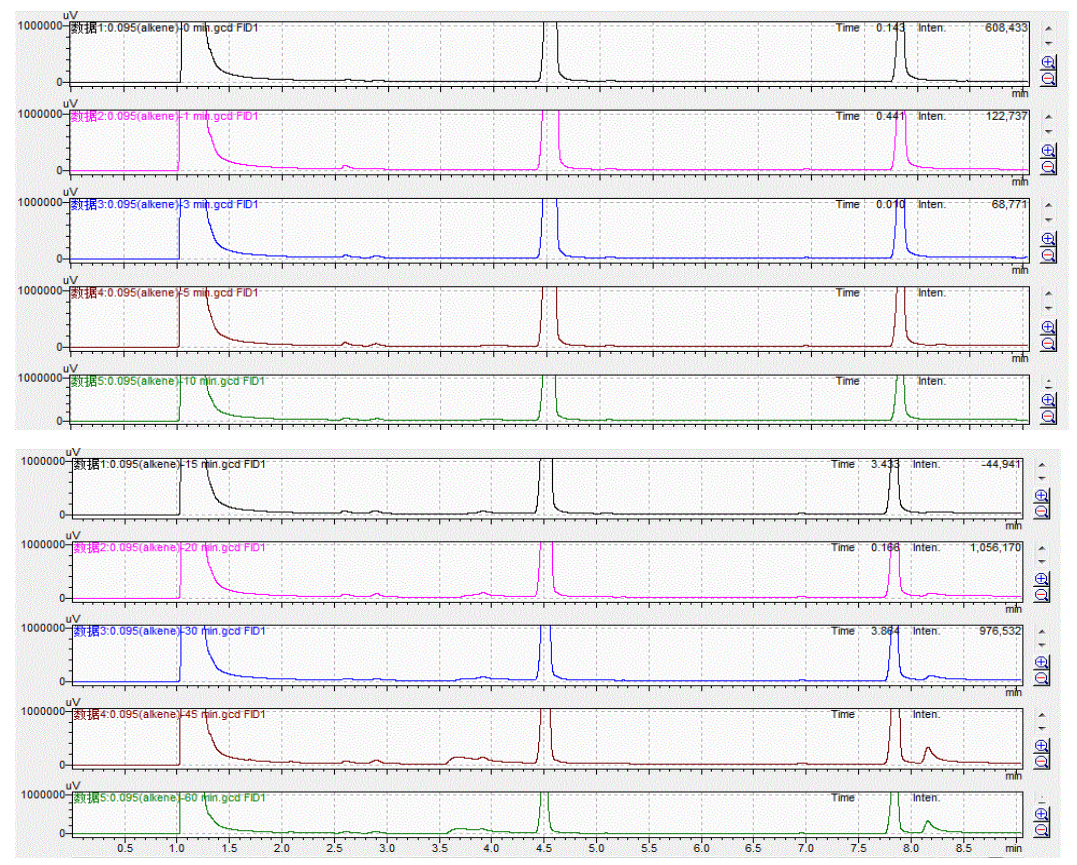

Figure S5. Conditions: [allyl benzyl ether] $=0.95 \mathrm{M}(0.95 \mathrm{mmol}, 141 \mathrm{mg})$, air (1 atm, balloon), ${ }^{t} \mathrm{BuONO}$ (20 mol \%, $20.6 \mathrm{mg}), \mathrm{Pd}\left(\mathrm{CH}_{3} \mathrm{CN}\right)_{2} \mathrm{Cl}_{2}(7.5 \mathrm{~mol} \%, 19.5 \mathrm{mg}), \mathrm{AcOH}(10 \mathrm{~mL}), 25{ }^{\circ} \mathrm{C}$, ethyl 3phenylpropionate $(0.25 \mathrm{mmol}, 45 \mathrm{mg})$ as internal standard.

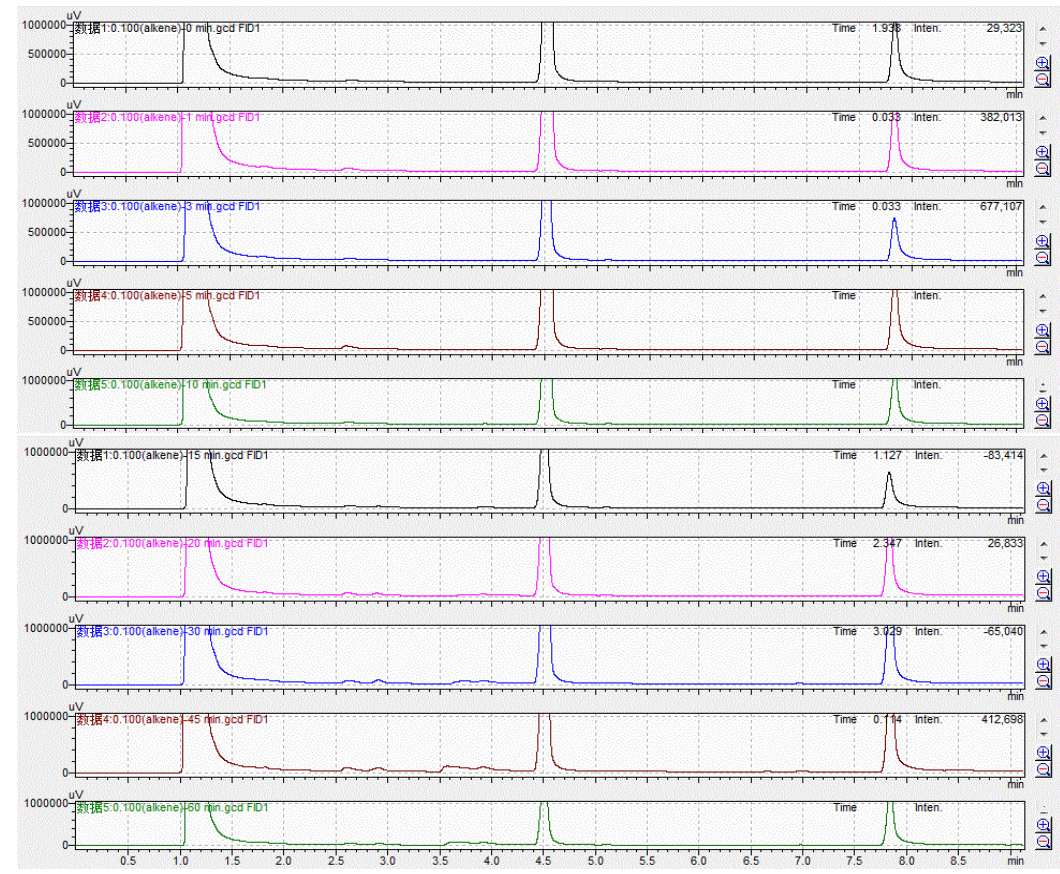

Figure S6. Conditions: [allyl benzyl ether] = $1.0 \mathrm{M}(1.0 \mathrm{mmol}, 148 \mathrm{mg})$, air (1 atm, balloon), ${ }^{t} \mathrm{BuONO}$ (20 mol \%, $20.6 \mathrm{mg}), \mathrm{Pd}\left(\mathrm{CH}_{3} \mathrm{CN}\right)_{2} \mathrm{Cl}_{2}(7.5 \mathrm{~mol} \%, 19.5 \mathrm{mg}), \mathrm{AcOH}(10 \mathrm{~mL}), 25{ }^{\circ} \mathrm{C}$, ethyl 3phenylpropionate $(0.25 \mathrm{mmol}, 45 \mathrm{mg})$ as internal standard. 


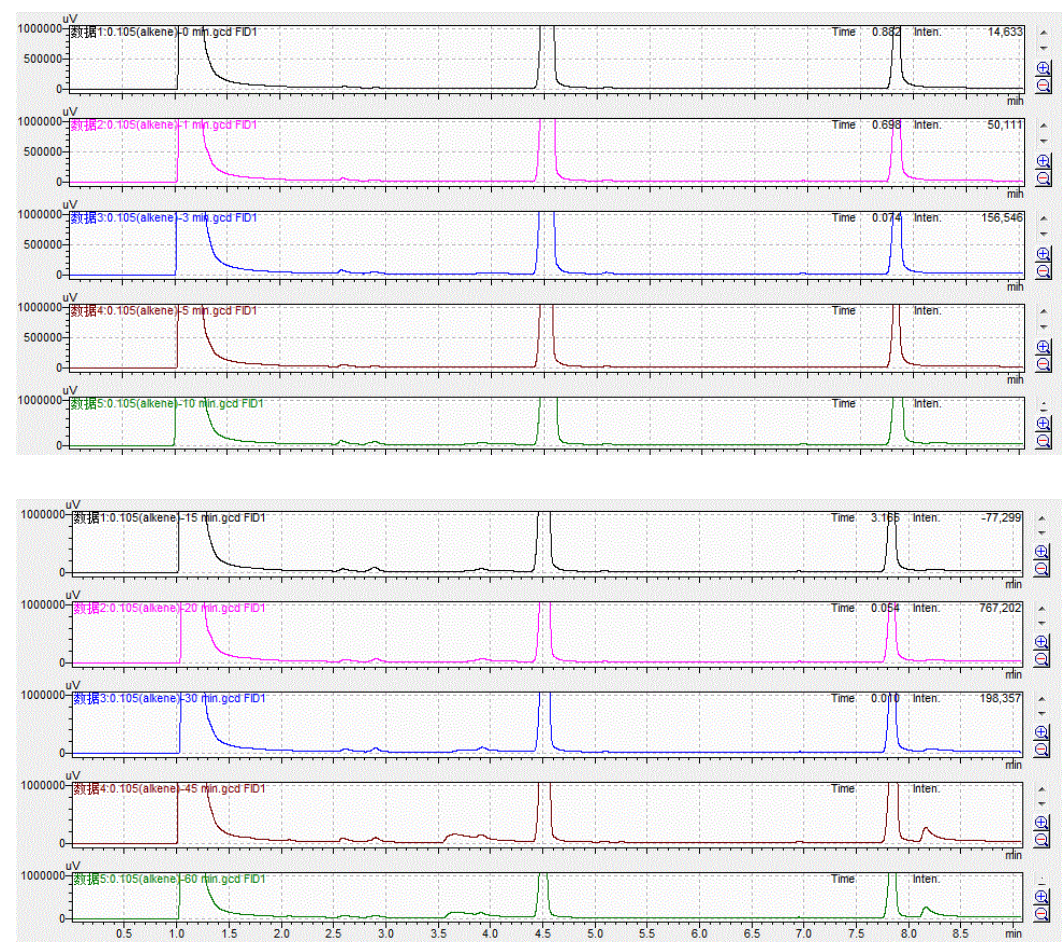

Figure S7. Conditions: [allyl benzyl ether] = $1.05 \mathrm{M}(1.05 \mathrm{mmol}, 156 \mathrm{mg})$, air ( $1 \mathrm{~atm}$, balloon $),{ }^{t} \mathrm{BuONO}$ (20 mol \%, $20.6 \mathrm{mg}), \operatorname{Pd}\left(\mathrm{CH}_{3} \mathrm{CN}\right)_{2} \mathrm{Cl}_{2}(7.5 \mathrm{~mol} \%, 19.5 \mathrm{mg}), \mathrm{AcOH}(10 \mathrm{~mL}), 25{ }^{\circ} \mathrm{C}$, ethyl 3phenylpropionate $(0.25 \mathrm{mmol}, 45 \mathrm{mg})$ as internal standard.

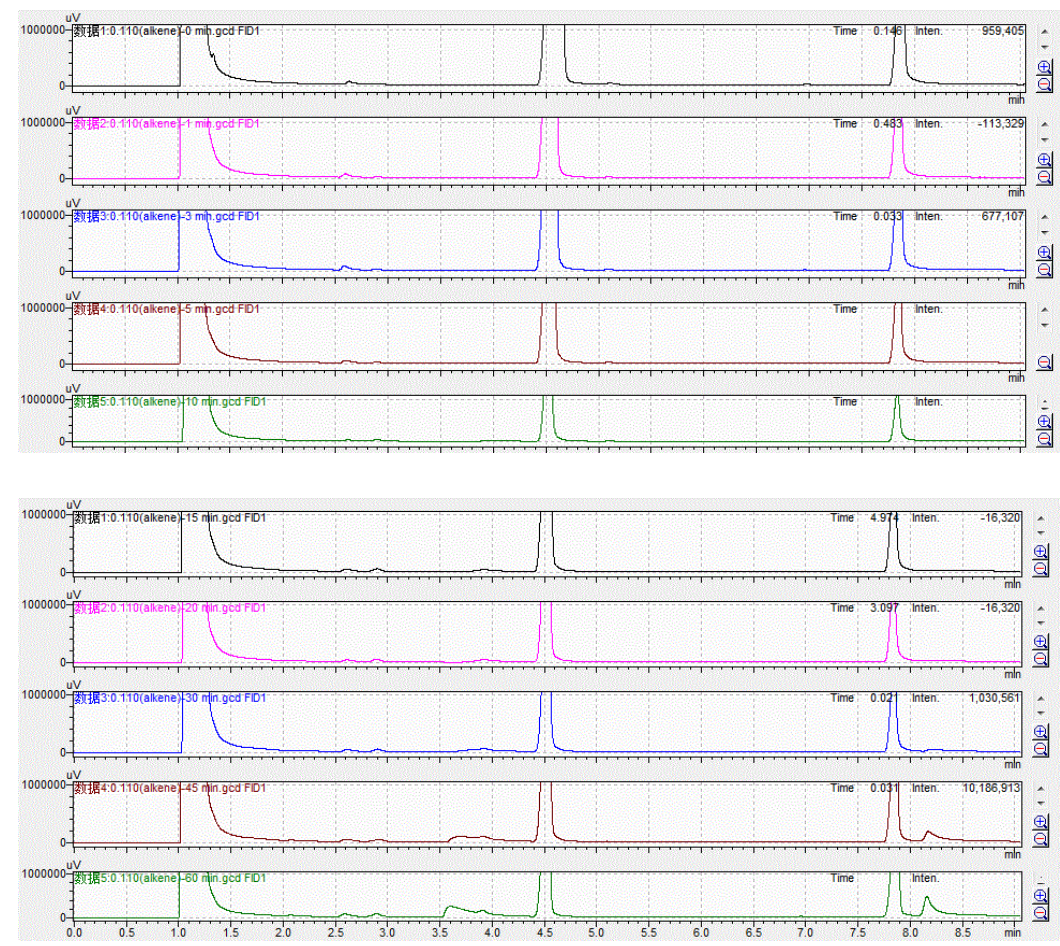

Figure S8. Conditions: [allyl benzyl ether] $=1.10 \mathrm{M}(1.10 \mathrm{mmol}, 163 \mathrm{mg})$, air (1 atm, balloon $),{ }^{t} \mathrm{BuONO}$ 
(20 mol \%, $20.6 \mathrm{mg}), \mathrm{Pd}\left(\mathrm{CH}_{3} \mathrm{CN}\right)_{2} \mathrm{Cl}_{2}(7.5 \mathrm{~mol} \%, 19.5 \mathrm{mg}), \mathrm{AcOH}(10 \mathrm{~mL}), 25{ }^{\circ} \mathrm{C}$, ethyl 3phenylpropionate $(0.25 \mathrm{mmol}, 45 \mathrm{mg})$ as internal standard.

Table S3. Dependence of allyl benzyl ether (M): [allyl phenyl ether] v.s. Time. ${ }^{a}$

\begin{tabular}{|c|c|c|c|c|}
\hline \multirow{3}{*}{ Time (min) } & \multicolumn{4}{|c|}{ allyl benzyl ether (M) } \\
\hline & S5 & S6 & S7 & S8 \\
\hline & $0.095(\mathrm{M})$ & $0.1(\mathrm{M})$ & $0.105(\mathrm{M})$ & $0.11(\mathrm{M})$ \\
\hline 0 & 0.095 & 0.1 & 0.105 & 0.11 \\
\hline 1 & 0.091637 & 0.09624 & 0.100947 & 0.106172 \\
\hline 3 & 0.087932 & 0.09234 & 0.0980855 & 0.102245 \\
\hline 5 & 0.0834195 & 0.08788 & 0.0923895 & 0.097955 \\
\hline 10 & 0.075905 & 0.07905 & 0.0828301 & 0.088231 \\
\hline 15 & 0.069483 & 0.07383 & 0.079065 & 0.082962 \\
\hline 20 & 0.062909 & 0.06565 & 0.0729435 & 0.078265 \\
\hline 30 & 0.0520315 & 0.05893 & 0.062223 & 0.064691 \\
\hline 45 & 0.0400235 & 0.04356 & 0.0486045 & 0.051557 \\
\hline 60 & 0.0298775 & 0.03204 & 0.0359205 & 0.038071 \\
\hline
\end{tabular}

${ }^{a}$ Conditions: Allyl benzyl ether (0.95-1.10 mmol, 140.8-163.0 mg), air (1 atm, balloon), ${ }^{t} \mathrm{BuONO}(20$ mol \%, $20.6 \mathrm{mg}), \mathrm{Pd}\left(\mathrm{CH}_{3} \mathrm{CN}\right)_{2} \mathrm{Cl}_{2}(7.5 \mathrm{~mol} \%, 19.5 \mathrm{mg}), \mathrm{AcOH}(10 \mathrm{~mL}), 25^{\circ} \mathrm{C}$, shielded with light, ethyl 3-phenylpropionate $(0.25 \mathrm{mmol}, 45 \mathrm{mg})$ as internal standard. 


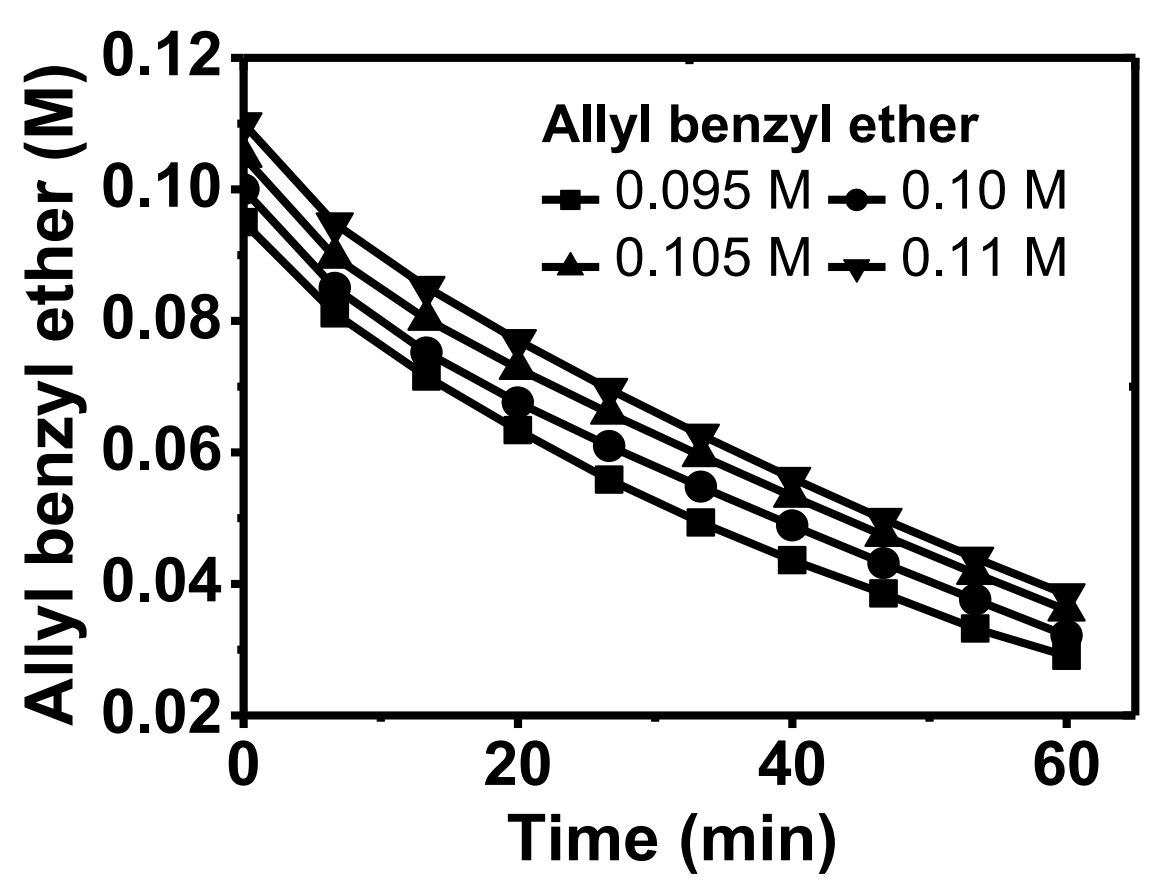

Figure 2. Dependence of the initial rate on allyl benzyl ether: Plots of [allyl benzyl ether] v.s. Time using allyl benzyl ether

\subsection{The dependence of the initial rate on [ $\left.{ }^{t} \mathrm{BuONO}\right]$.}

General procedure: Allyl benzyl ether $(1.0 \mathrm{mmol}, 148 \mathrm{mg})$ was weighed directly into a $50 \mathrm{~mL}$ roundbottomed flask under the atmosphere of air (1 atm, balloon) condition, AcOH (10 mL) and ethyl 3phenylpropionate $\left(0.25 \mathrm{mmol}, 45 \mathrm{mg}\right.$ ) (as internal standard) were added, fallowed with $\mathrm{Pd}\left(\mathrm{CH}_{3} \mathrm{CN}\right)_{2} \mathrm{Cl}_{2}$ (7.5 mol \%, $19.5 \mathrm{mg})$ and ${ }^{t} \mathbf{B u O N O}(\mathbf{1 5 . 0 - 2 2 . 5} \mathrm{mol} \%, \mathbf{1 5 . 5 - 2 3 . 2} \mathbf{m g})$ were added at the same time. The reaction flask was shielded with light using aluminum foil, the resulting reaction mixture was stirred with the oil bath and the indoor temperature at $25^{\circ} \mathrm{C}$. At each sampling time $50 \mu \mathrm{L}$ reaction mixture was extracted and examined by GC. [allyl benzyl ether] was determined using ethyl 3-phenylpropionate as internal standard and the results were demonstrated in Table S4.

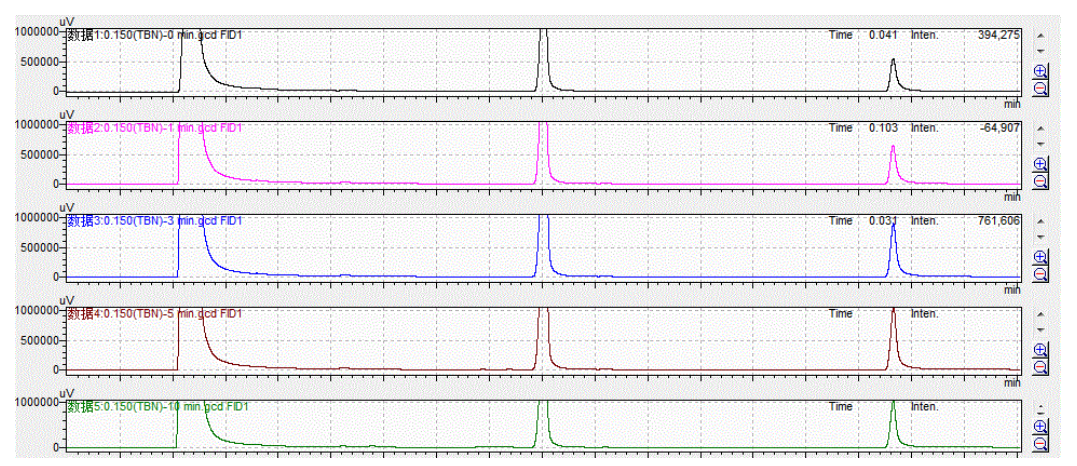




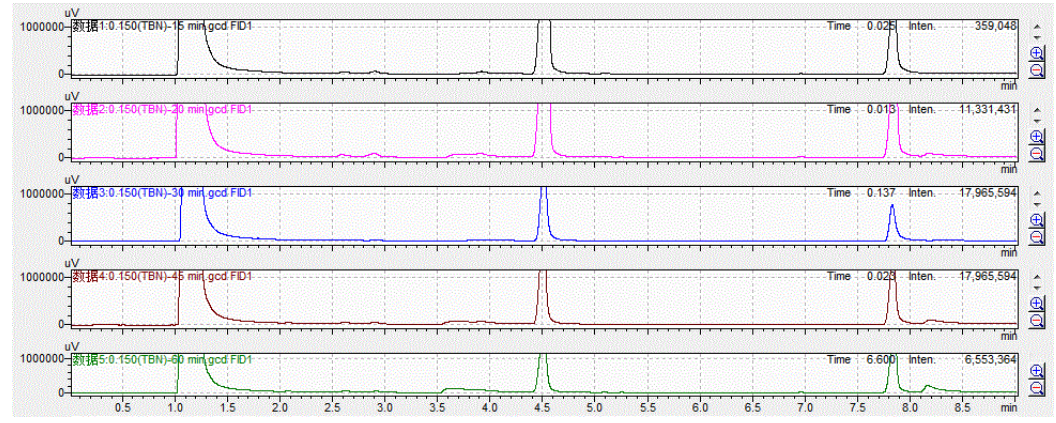

Figure S9. Conditions: [allyl benzyl ether] $=0.1 \mathrm{M}(1.0 \mathrm{mmol}, 148 \mathrm{mg})$, air (1 atm, balloon), ${ }^{t} \mathrm{BuONO}$ (15.0 mol \%, $16 \mathrm{mg}), \mathrm{Pd}\left(\mathrm{CH}_{3} \mathrm{CN}\right)_{2} \mathrm{Cl}_{2}(7.5 \mathrm{~mol} \%, 19.5 \mathrm{mg}), \mathrm{AcOH}(10 \mathrm{~mL}), 25{ }^{\circ} \mathrm{C}$, ethyl 3phenylpropionate $(0.25 \mathrm{mmol}, 45 \mathrm{mg})$ as internal standard.

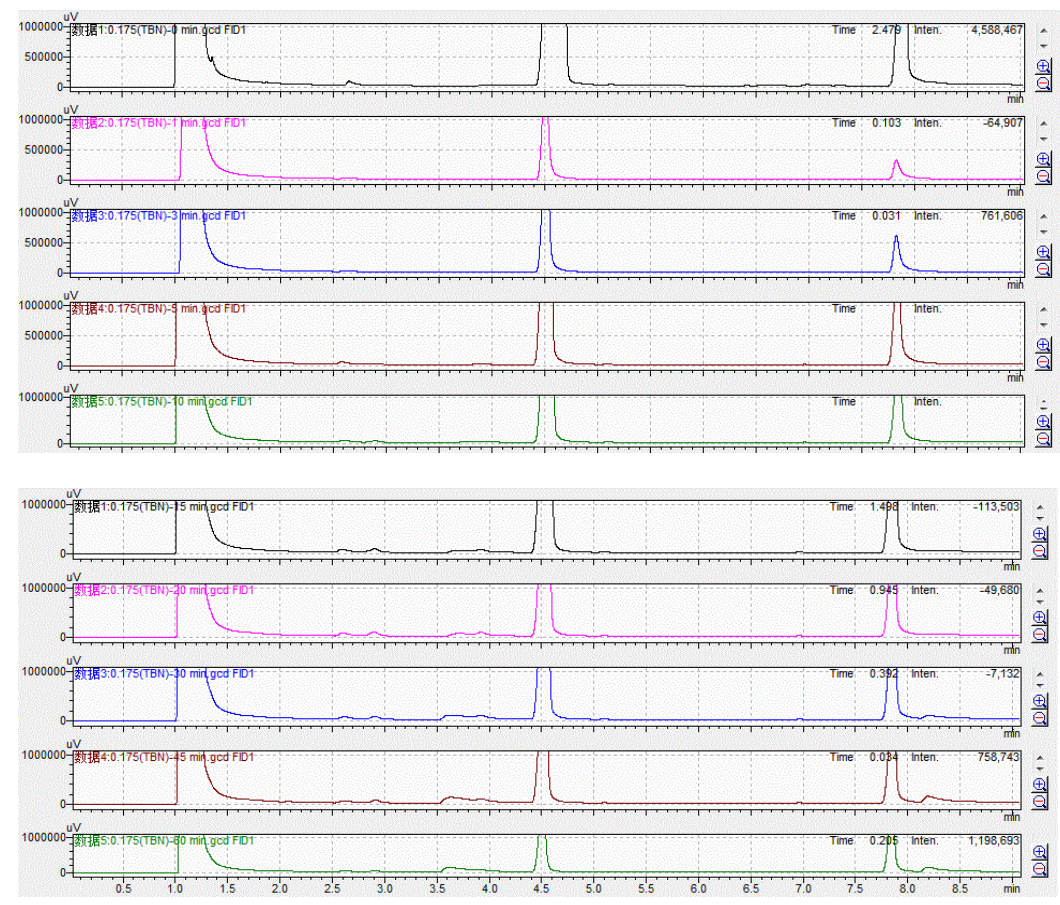

Figure S10. Conditions: [allyl benzyl ether] = 0.1 M (1.0 mmol, $148 \mathrm{mg})$, air (1 atm, balloon), ${ }^{\boldsymbol{t}} \boldsymbol{B u O N O}$ (17.5 mol \%, $18 \mathrm{mg}), \operatorname{Pd}\left(\mathrm{CH}_{3} \mathrm{CN}\right)_{2} \mathrm{Cl}_{2}(7.5 \mathrm{~mol} \%, 19.5 \mathrm{mg})$, $\mathrm{AcOH}(10 \mathrm{~mL}), 25{ }^{\circ} \mathrm{C}$, ethyl 3phenylpropionate $(0.25 \mathrm{mmol}, 45 \mathrm{mg})$ as internal standard.

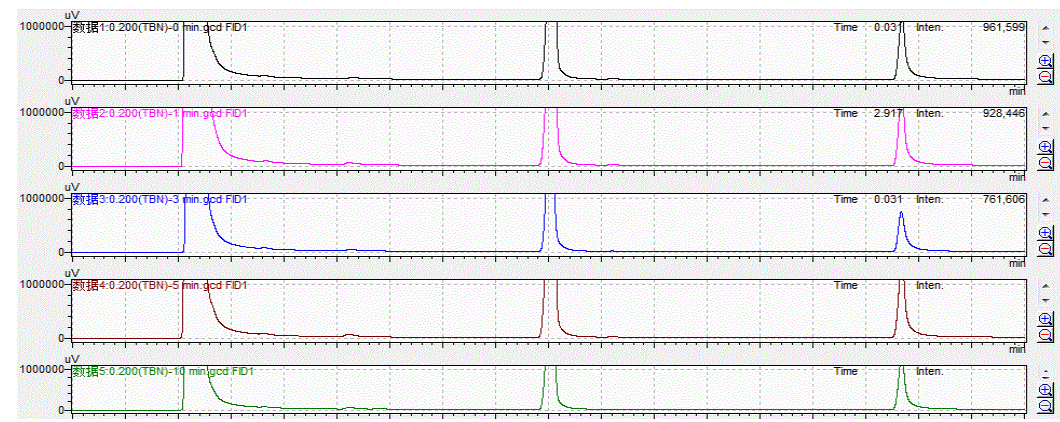




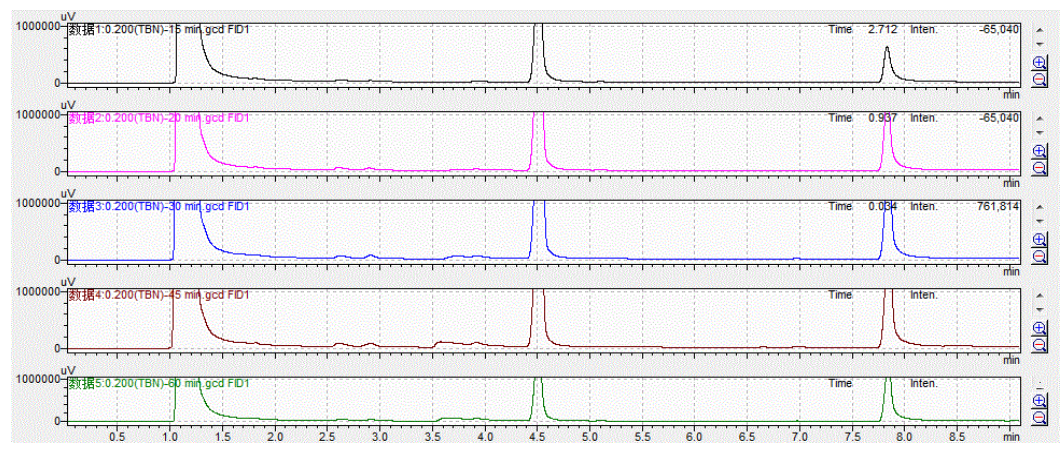

Figure S11. Conditions: [allyl benzyl ether] $=0.1 \mathrm{M}(1.0 \mathrm{mmol}, 148 \mathrm{mg})$, air (1 atm, balloon $),{ }^{\boldsymbol{t}} \mathrm{BuONO}$ (20.0 mol \%, $20.6 \mathrm{mg}), \mathrm{Pd}\left(\mathrm{CH}_{3} \mathrm{CN}\right)_{2} \mathrm{Cl}_{2}(7.5 \mathrm{~mol} \%, 19.5 \mathrm{mg})$, AcOH $(10 \mathrm{~mL}), 25{ }^{\circ} \mathrm{C}$, ethyl 3phenylpropionate $(0.25 \mathrm{mmol}, 45 \mathrm{mg})$ as internal standard.

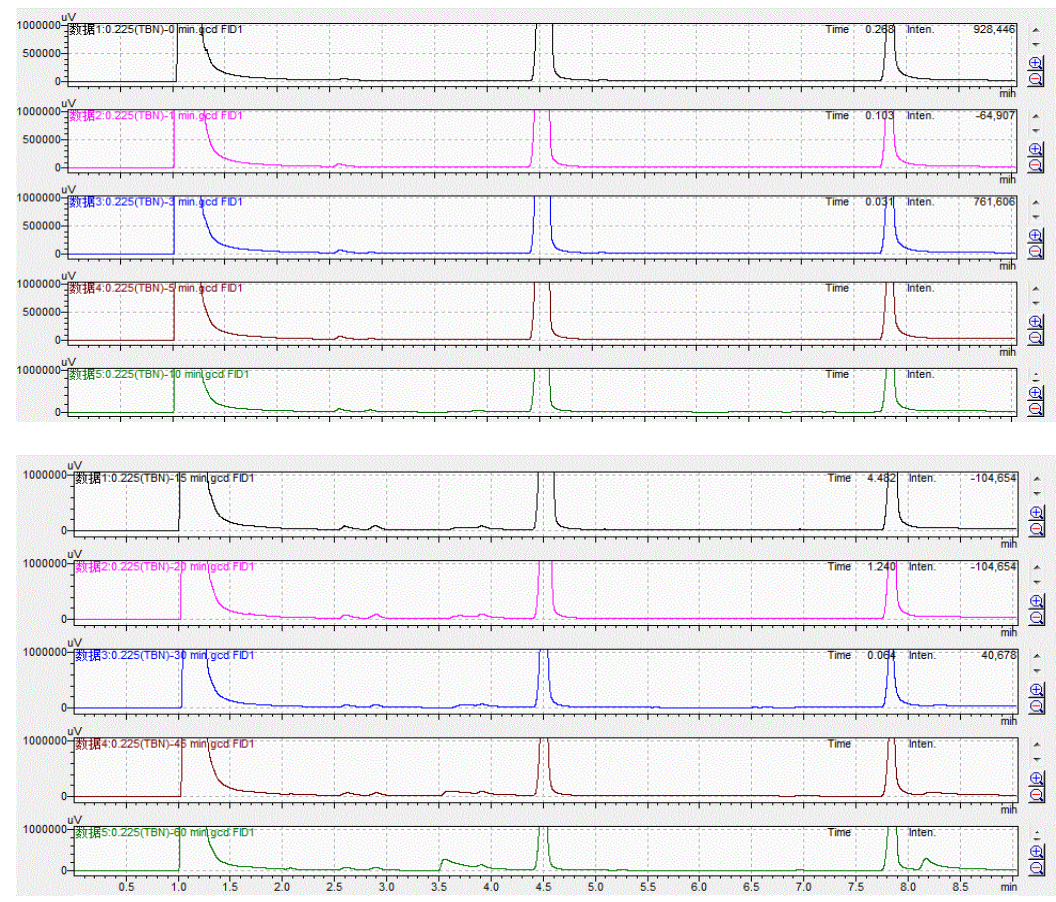

Figure S12. Conditions: [allyl benzyl ether] = 0.1 M (1.0 mmol, $148 \mathrm{mg})$, air (1 atm, balloon), ${ }^{\boldsymbol{t}}$ BuONO (22.5 mol \%, $23 \mathrm{mg}), \mathrm{Pd}\left(\mathrm{CH}_{3} \mathrm{CN}\right)_{2} \mathrm{Cl}_{2}(7.5 \mathrm{~mol} \%, 19.5 \mathrm{mg}), \mathrm{AcOH}(10 \mathrm{~mL}), 25{ }^{\circ} \mathrm{C}$, ethyl 3phenylpropionate $(0.25 \mathrm{mmol}, 45 \mathrm{mg})$ as internal standard.

Table S4. Dependence of ${ }^{t} \mathrm{BuONO}(\mathrm{mol} \%)$ : [allyl benzyl ether] v.s. Time. ${ }^{a}$

\begin{tabular}{lllll}
\hline \multirow{2}{*}{ Time (min) } & \multicolumn{4}{l}{ allyl benzyl ether $(\mathrm{M})$} \\
\cline { 2 - 5 } & $\mathrm{S} 9$ & $\mathrm{~S} 10$ & $\mathrm{~S} 11$ & $\mathrm{~S} 12$ \\
\hline & $15.0 \mathrm{~mol} \mathrm{\%}$ & $17.5 \mathrm{~mol} \%$ & $20.0 \mathrm{~mol} \%$ & $22.5 \mathrm{~mol} \%$ \\
\hline 0 & 0.1 & 0.1 & 0.1 & 0.1 \\
\hline
\end{tabular}




\begin{tabular}{lllll}
\hline 1 & 0.09636 & 0.09570 & 0.09624 & 0.09512 \\
\hline 3 & 0.09214 & 0.09179 & 0.09234 & 0.09110 \\
\hline 5 & 0.08814 & 0.08660 & 0.08788 & 0.08735 \\
\hline 10 & 0.08194 & 0.07827 & 0.07905 & 0.07819 \\
\hline 15 & 0.07559 & 0.07339 & 0.07383 & 0.07177 \\
\hline 30 & 0.06581 & 0.06478 & 0.06565 & 0.06270 \\
\hline 60 & 0.06119 & 0.05721 & 0.05893 & 0.05637 \\
\hline
\end{tabular}

${ }^{a}$ Conditions: Allyl benzyl ether ( $\left.1 \mathrm{mmol}, 148 \mathrm{mg}\right)$, air (1 atm, balloon), ${ }^{t}$ BuONO (15.0-22.5 mol \%, 15.5$23.2 \mathrm{mg}), \mathrm{Pd}\left(\mathrm{CH}_{3} \mathrm{CN}\right)_{2} \mathrm{Cl}_{2}(7.5 \mathrm{~mol} \%, 19.5 \mathrm{mg}), \mathrm{AcOH}(10 \mathrm{~mL}), 25{ }^{\circ} \mathrm{C}$, shielded with light, ethyl 3phenylpropionate $(0.25 \mathrm{mmol}, 45 \mathrm{mg})$ as internal standard.

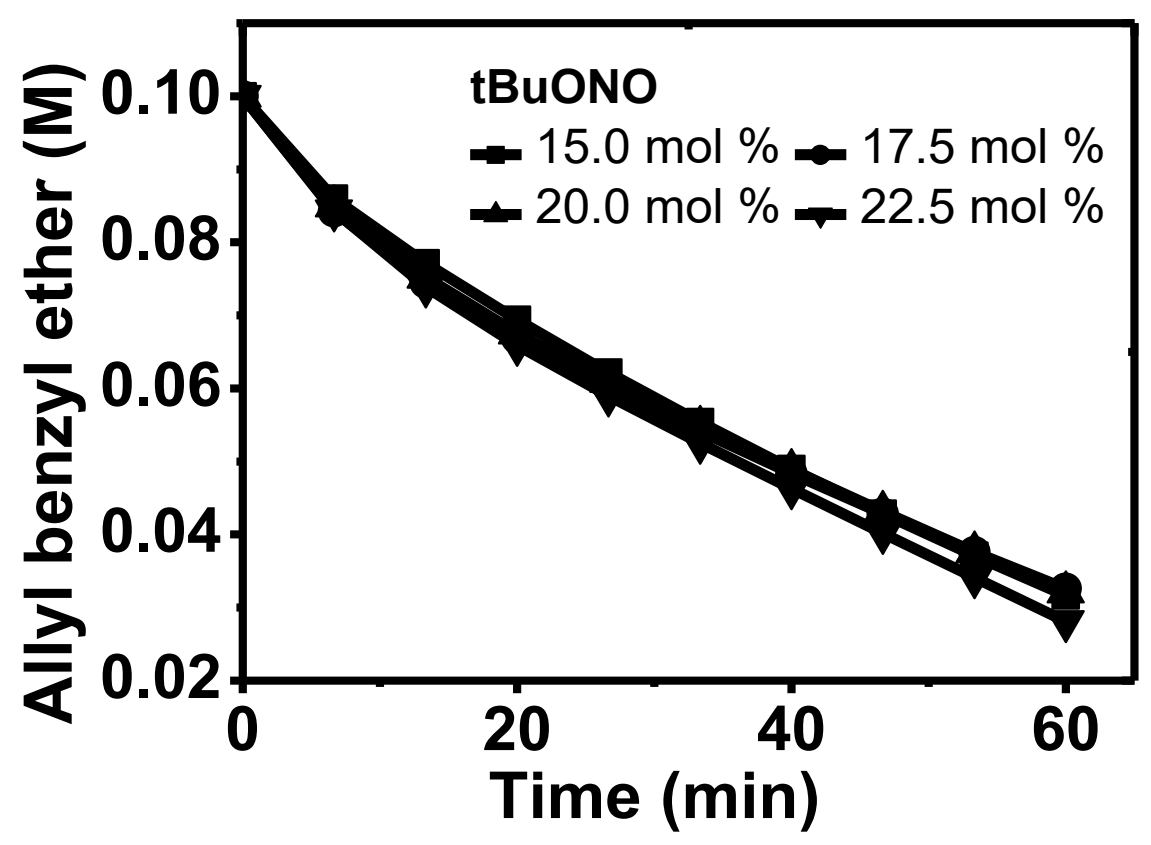

Figure 3. Dependence of the initial rate on $\left[{ }^{t} \mathbf{B u O N O}\right.$ ]: Plots of [allyl benzyl ether] v.s. Time using ${ }^{t} \mathrm{BuONO}$ 


\section{NMR monitoring experiments}<smiles>C=CC(=O)c1ccccc1</smiles>

$1 \mathrm{i}$

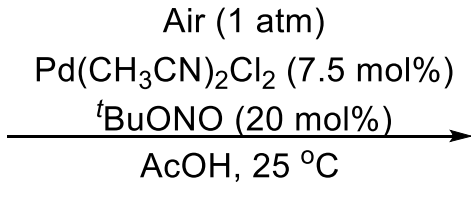

$\mathrm{AcOH}, 25^{\circ} \mathrm{C}$<smiles>CC(=O)OC(CO)CC(=O)c1ccccc1</smiles>

3iA<smiles>CC(=O)OCC(O)C(=[18O])C(=O)c1ccccc1</smiles>

$3 i B$

$\mathrm{Pd}\left(\mathrm{CH}_{3} \mathrm{CN}\right)_{2} \mathrm{Cl}_{2}(7.5 \mathrm{~mol} \%, 19.5 \mathrm{mg})$ was weighed directly into a $50 \mathrm{~mL}$ round-bottomed flask charged with a stir bar and dried under high vacuum for 15 mins, purge air 3 times. Under an atmosphere of air (1 atm, balloon) $10 \mathrm{~mL}$ of $\mathrm{AcOH},{ }^{t} \mathrm{BuONO}(20 \mathrm{~mol} \%, 20.6 \mathrm{mg})$ and ethyl 3-phenylpropionate (0.25 mmol, $45 \mathrm{mg}$ ) (as internal standard) were added and stirred at the oil bath and the indoor temperature at $25^{\circ} \mathrm{C}$. 1-phenylundec-10-en-1-one ( $1 \mathrm{mmol}, 244 \mathrm{mg}$ ) was then added and the reaction vessel was shielded with light using aluminum foil, the resulting reaction mixture was stirred at $25{ }^{\circ} \mathrm{C}$. At each sampling time 30 $\mu \mathrm{L}$ reaction mixture was extracted and examined by ${ }^{1} \mathrm{H}$ NMR. 1-phenylundec-10-en-1-one [1i] and Monoacetylation product $[\mathbf{3 i A}]$ and [3iB] were determined by ${ }^{1} \mathrm{H}$ NMR using ethyl 3-phenylpropionate as internal standard.

\begin{tabular}{llll}
\hline \multirow{2}{*}{ Time $(\min )$} & monoacetylation product & allyl benzyl ether \\
\cline { 2 - 4 } & $\mathbf{3 i A}(\%)$ & $\mathbf{3 i B}(\%)$ & $\mathbf{1 i}(\%)$ \\
\hline 0 & 0 & 0 & 100 \\
\hline 25 & 9 & 5 & 84 \\
\hline 60 & 18 & 14 & 61 \\
\hline 90 & 23 & 18 & 35 \\
\hline 120 & 34 & 21 & 12 \\
\hline 180 & 42 & 27 & 0 \\
\hline 240 & 44 & 32 & 0 \\
\hline 300 & 48 & 34 & 0 \\
\hline
\end{tabular}

${ }^{a}$ Conditions: 1-phenylundec-10-en-1-one (1 mmol, $\left.148 \mathrm{mg}\right)$, air (1 atm, balloon), ${ }^{t} \mathrm{BuONO}(20 \mathrm{~mol} \%$, $20.6 \mathrm{mg}), \mathrm{Pd}\left(\mathrm{CH}_{3} \mathrm{CN}\right)_{2} \mathrm{Cl}_{2}(7.5 \mathrm{~mol} \%, 19.5 \mathrm{mg}), \mathrm{AcOH}(10 \mathrm{~mL}), 25{ }^{\circ} \mathrm{C}$, ethyl 3-phenylpropionate $(0.25$ mmol, $45 \mathrm{mg}$ ) as internal standard.

NMR monitoring experiments for $\mathrm{Pd}-\mathrm{Cu}-\mathrm{Ag}-\mathrm{NO}_{2}$ system without $\mathrm{Ac}_{2} \mathrm{O}$ : 


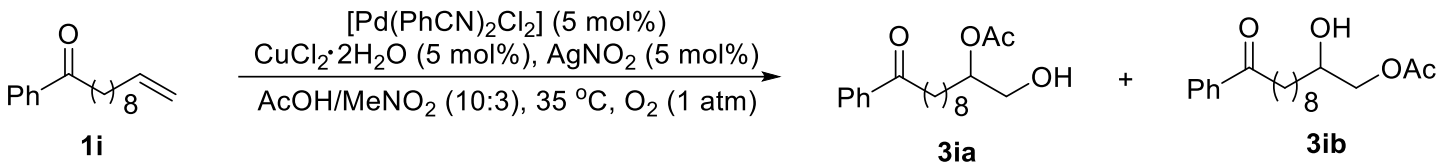

$\mathrm{PdCl}_{2}(\mathrm{PhCN})_{2}(0.05 \mathrm{mmol}, 19.2 \mathrm{mg}), \mathrm{CuCl}_{2} \cdot 2 \mathrm{H}_{2} \mathrm{O}(0.05 \mathrm{mmol}, 8.5 \mathrm{mg})$ and $\mathrm{AgNO}_{2}(0.02 \mathrm{mmol}, 8.5 \mathrm{mg})$ were weighed into a $50 \mathrm{~mL}$ round-bottomed flask with a stir bar. The flask was sparged for 1 minute with oxygen (1 atm, balloon). $\mathrm{AcOH}$ and $\mathrm{MeNO}_{2}$ were premixed in a 10:3 ratio and sparged with oxygen for 2 minutes. The oxygenated solvent mixture was then added to the vial $(10 \mathrm{~mL})$, followed by ethyl 3 phenylpropionate $(0.25 \mathrm{mmol}, 45 \mathrm{mg})$ (as internal standard) and the alkene substrate $(1.0 \mathrm{mmol})$ via syringe. After an additional 15 seconds of sparging with oxygen, the reaction vessel was shielded with light using aluminum foil. The reaction was then allowed to stir at $35^{\circ} \mathrm{C}$ under an atmosphere of oxygen (balloon). At each sampling time $30 \mu$ Lreaction mixture was extracted and examined by ${ }^{1} \mathrm{H}$ NMR. Starting material [1i] , monocetylation product [3ia], [3ib] and [3ia] $+[3 \mathbf{3 i b}]$ were determined by ${ }^{1} \mathrm{HNMR}$ using ethyl 3-phenylpropionate as internal standard and the results were demonstrated in the following Table and Figure.

\begin{tabular}{llll} 
Time (min) & $\mathbf{1 i}(\boldsymbol{\%})$ & 3ia $(\boldsymbol{\%})$ & 3ib (\%) \\
\hline 0 min & 100 & 0 & 0 \\
\hline 20 min & 75 & 13 & 4 \\
\hline 40 min & 53 & 32 & 10 \\
\hline 60 min & 32 & 41 & 18 \\
\hline 90 min & 12 & 48 & 31 \\
\hline 120 min & 3 & 52 & 32 \\
\hline 180 min & 0 & 53 & 34 \\
\hline 240 min & 0 & 54 & \\
\hline
\end{tabular}

\section{NMR monitoring experiments for $\mathrm{Pd}-\mathrm{Cu}-\mathrm{Ag}-\mathrm{NO}_{2}$ system with $\mathrm{Ac}_{2} \mathrm{O}$ :}




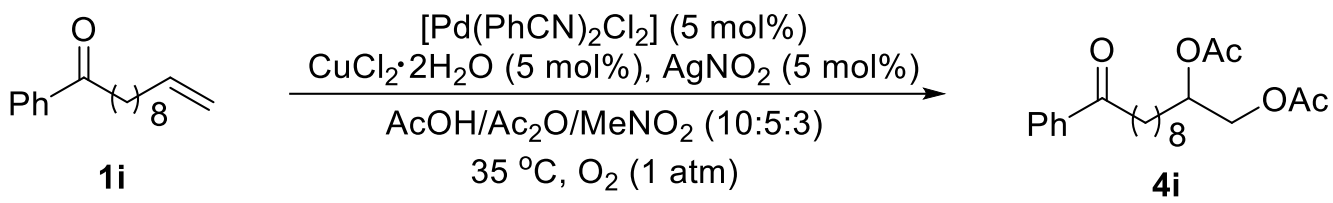

$\mathrm{PdCl}_{2}\left(\mathrm{PhCN}_{2}(0.05 \mathrm{mmol}, 19.2 \mathrm{mg}), \mathrm{CuCl}_{2} \cdot 2 \mathrm{H}_{2} \mathrm{O}(0.05 \mathrm{mmol}, 8.5 \mathrm{mg})\right.$ and $\mathrm{AgNO}_{2}(0.02 \mathrm{mmol}, 8.5 \mathrm{mg})$ were weighed into a $50 \mathrm{~mL}$ round-bottomed flask with a stir bar. The flask was sparged for 1 minute with oxygen (1 atm, balloon). $\mathrm{AcOH}, \mathrm{Ac}_{2} \mathrm{O}$ and $\mathrm{MeNO}_{2}$ were premixed in a 10:5:3 ratio and sparged with oxygen for 2 minutes. The oxygenated solvent mixture was then added to the vial $(16 \mathrm{~mL})$, followed by ethyl 3-phenylpropionate $(0.25 \mathrm{mmol}, 45 \mathrm{mg})$ (as internal standard) and the alkene substrate $(1.0 \mathrm{mmol})$ via syringe. After an additional 15 seconds of sparging with oxygen, the reaction vessel was shielded with light using aluminum foil. The reaction was then allowed to stir at $35^{\circ} \mathrm{C}$ under an atmosphere of oxygen (balloon). At each sampling time $30 \mu \mathrm{L}$ reaction mixture was extracted and examined by ${ }^{1} \mathrm{H}$ NMR. Starting material [1i] and diacetylation product [4i] were determined by ${ }^{1}$ HNMR using ethyl 3-phenylpropionate as internal standard and the results were demonstrated in the following Table and Figure.

\begin{tabular}{lll} 
Time (min) & $\mathbf{1 a}(\boldsymbol{\%})$ & $\mathbf{3 a}(\boldsymbol{\%})$ \\
\hline 0 min & 100 & 6 \\
\hline 45 min & 72 & 14 \\
\hline 90 min & 60 & 28 \\
\hline 150 min & 42 & 40 \\
\hline 240 min & 32 & 45 \\
\hline 300 min & 24 & 49 \\
\hline 420 min & 10 & 54 \\
\hline 540 min & 0 & 55 \\
\hline 660 min & 0 & 56 \\
\hline 810 min & 0 & \\
\hline
\end{tabular}




\section{References}

(1) Liu, R.; Lu, Z.-H.; Hu, X.-H.; Li, J.-L.; Yang, X.-J. Org. Lett. 2015, 17, 1489.

(2) Thuong, M. B. T.; Mann, A.; Wagner, A. Chem. Commun. 2012, 48, 434.

(3) Gutierrez, A. C.; Jamison, T. F. Org. Lett. 2011, 13, 6414.

(4) Fujii, S.; Lehn, J. M. Angew. Chem., Int. Ed. 2009, 48, 7635.

(5) Fash, D. M.; Khdour, O. M.; Sahdeo, S. J.; Goldschmidt, R.; Jaruvangsanti, J.; Dey, S.; Arce, P. M.;

Collin, V. C.; Cortopassi, G. A.; Hecht. S. M. Bioorg. Med. Chem, 2013, 21, 2346.

(6) Bérubé, M.; Poirier, D. Can. J. Chem, 2009, 87, 1180.

(7) Kliman, L. T.; Mlynarski, S. N.; Morken, J. P. J. Am. Chem. Soc. 2009, 131, 13210.

(8) Han, Z.; Rong, L.; Wu, J.; Zhang, L.; Wang, Z.; Ding, K. Angew. Chem., Int. Ed. 2012, 51, 13041.

(9) Yao, Q. Org. Lett. 2002, 4, 2197.

(10) Prat, I.; Font, D.; Company, A.; Junge, K.; Ribas, X.; Beller, M.; Costas, M. Adv. Synth. Cata. 2013, $355,947$.

(11) Furukawa, K.; Shibuya, M.; Yamamoto, Y. Org. Lett. 2015, 17, 2282.

(12) Cravotto, G.; Gaudino, E. C.; Barge, A.; Binello, A.; Albertino, A.; Aghemo C. Nat Prod Res, 2010, 24,428 .

(13) Wickens, Z. K.; Guzmán, P. E.; Grubbs. R. H. Angew. Chem., Int. Ed. 2015, 54, 236.

(14) Ramesh, P.; Reddy, V. L. N.; Venugopal, D.; Subrahmanyam, M.; Venkateswarlu, Y. Synth. Commun, 2001, 31, 2599.

(15) Kang, Y.-B.; Gade, L. H. J. Am. Chem. Soc. 2011, 133, 3658. 


\section{NMR spectra}

$100^{\circ} 0^{-}$

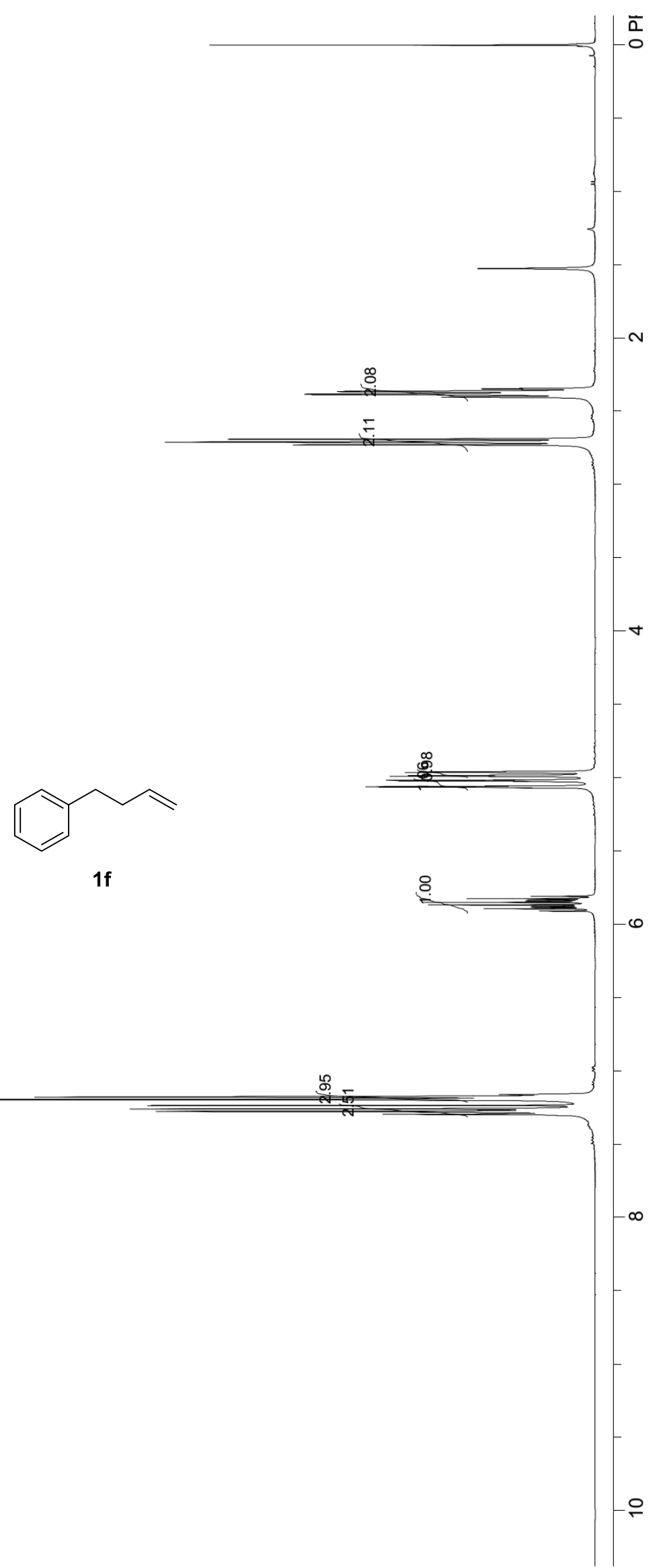

$\varepsilon \succ \varepsilon \cdot 2$

$\angle \downarrow \varepsilon^{\prime} 乙$
$\varepsilon 9 \varepsilon^{\prime} 乙$

乙8६ 2

$20 t^{2} \mathrm{C}$

$689^{\circ} \mathrm{Z}$

OLL'Z

$62 L ' Z$

896.5

$196^{\circ} \circ$

$996^{\circ} \circ$

$896^{\circ} t$

$\varepsilon 86^{\circ} t$

986

$166^{\circ} t$

6109

$\varepsilon Z 0^{\circ} \mathrm{S}$

$\angle Z O^{\circ} G$

$\angle 90^{\circ} 9$

$190^{\circ} \mathrm{S}$

$990 \mathrm{~s}$

$0 \angle 0^{\circ} 9$

018.

9Z8. 9

ZS8. 9

$898^{\circ} 9$

เL6.9

$\varepsilon 9 L^{\circ} L$

$99 L^{\circ} \mathrm{L}$

$\angle 6 L L$

$\angle \varepsilon Z Z^{\prime} L$

I9Z' L

$19 Z^{\prime} L$

$89 Z^{\prime} L$

$86 Z^{\prime} L$ 
$000^{\circ} 0^{-}$

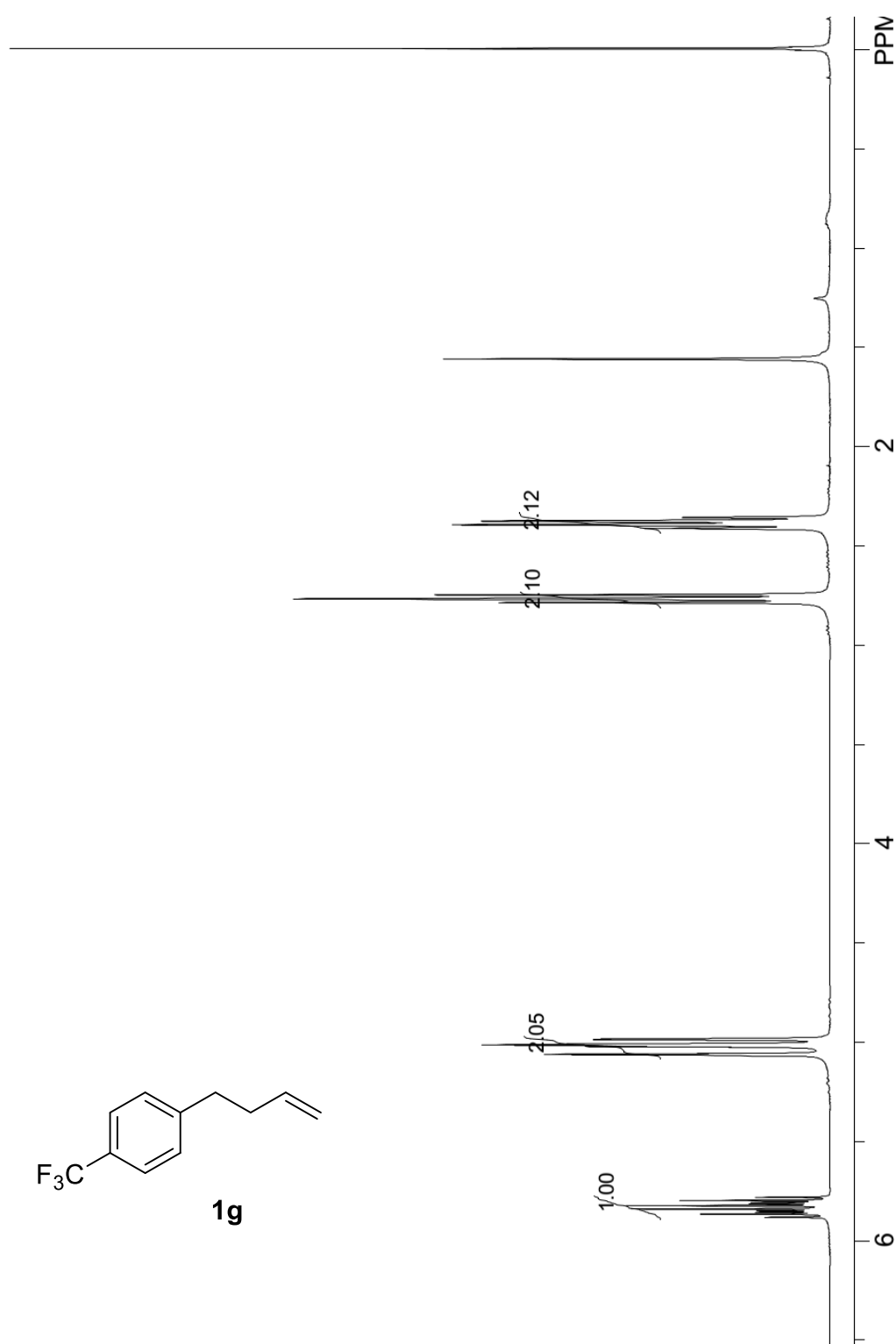

$\varepsilon 86^{\circ} t$

$\angle 86^{\circ} t$

ELO'

ZZO'

090.9

$790^{\circ} \mathrm{s}$

690 '

$18 L^{\circ} \mathrm{C}$

$86 L^{\circ} \mathrm{G}$

$\angle 08^{\circ} 9$

나 9

$\varepsilon Z 8^{\circ} \mathrm{G}$

$0+89$

678.9

$\angle 98^{\circ} 9$

$998^{\circ} \mathrm{9}$

Z88. 9

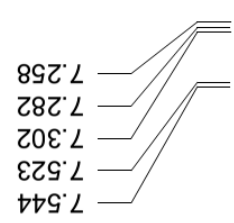




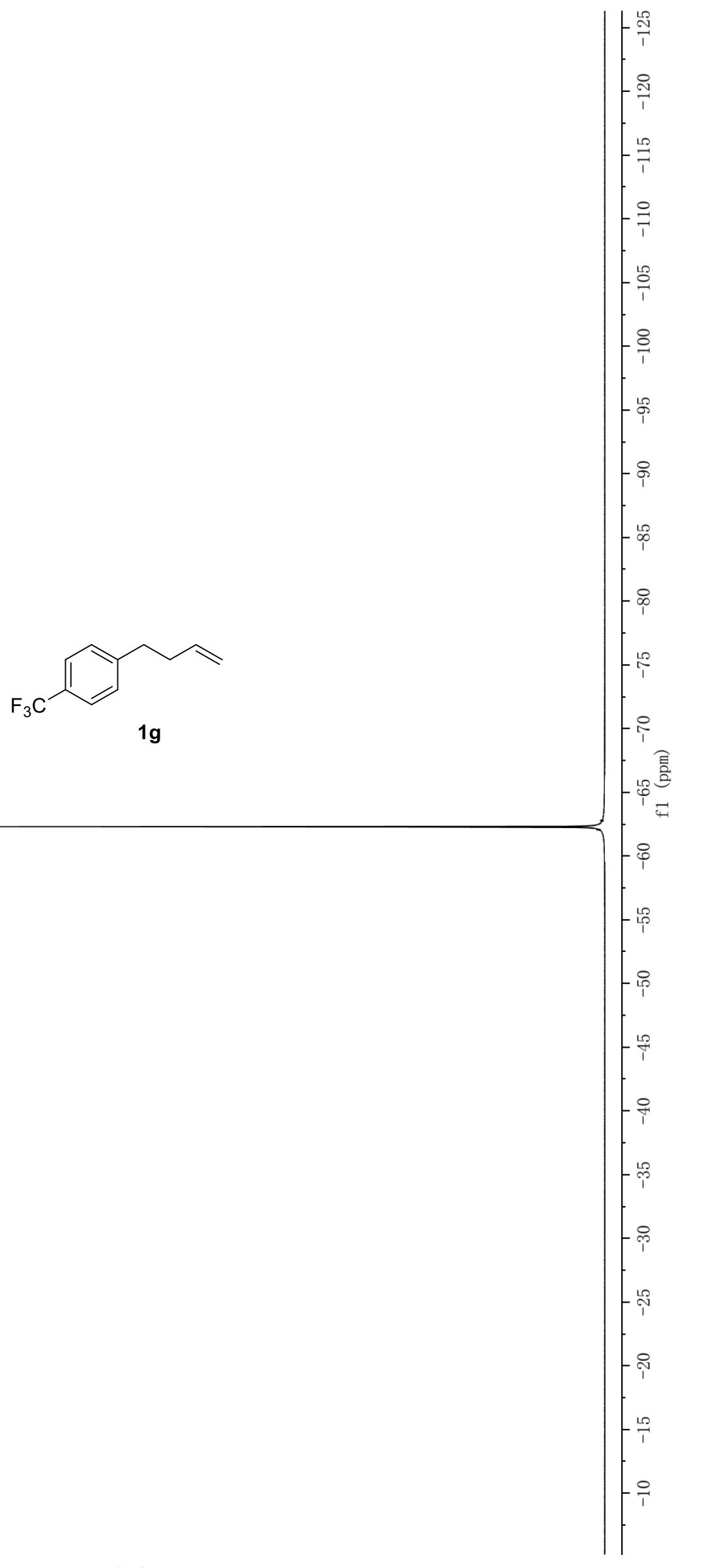

S-35 

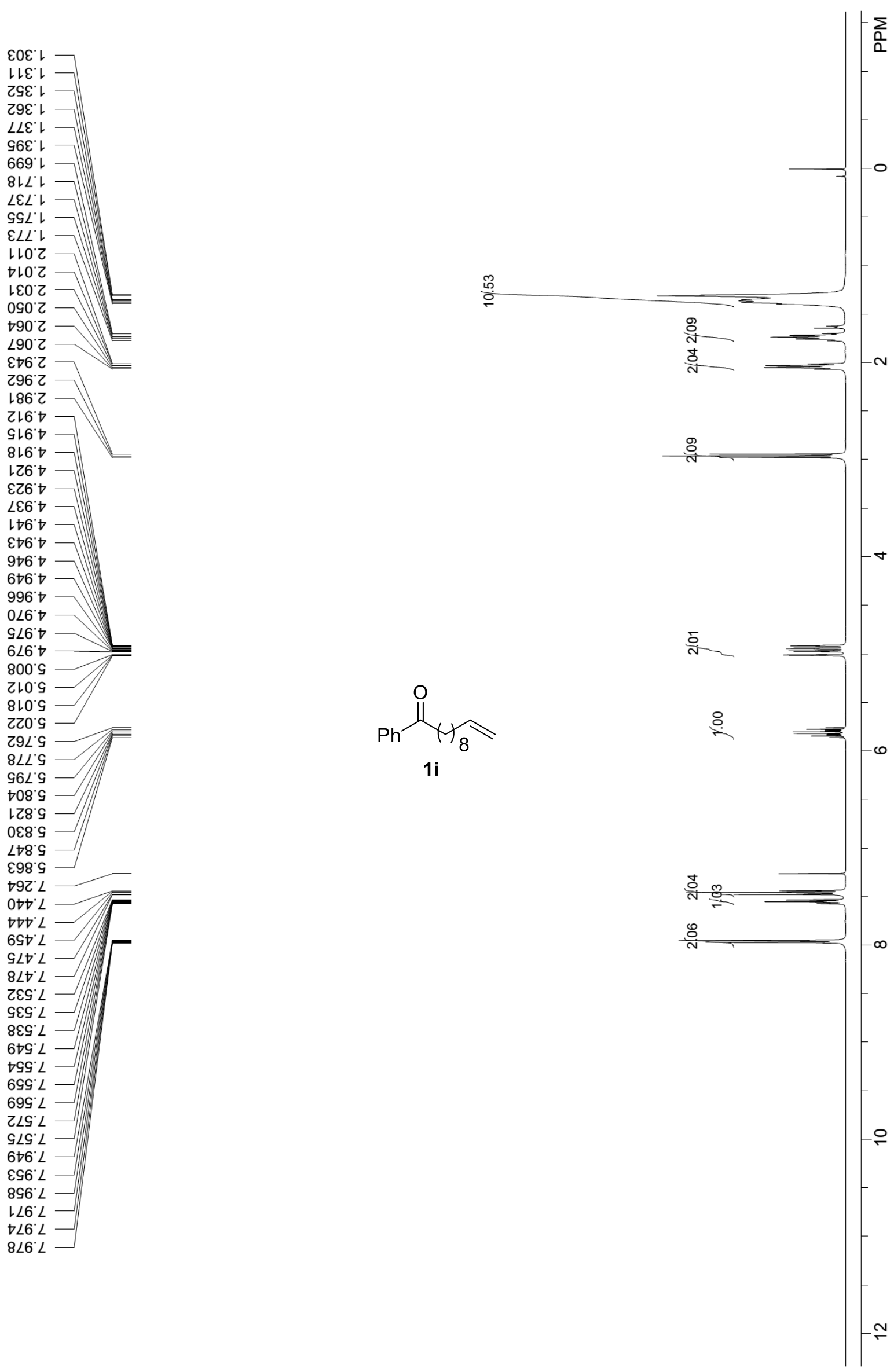
$0 \angle 9^{\circ} \nabla \tau$

乙L乙゙6乙

与6ย 62

979.62

$\nabla 99^{\circ} 62$

$S \nabla L ' 6 Z$

$60 L^{\circ} \downarrow \varepsilon$

$\varepsilon \varepsilon 6^{\circ} 8 \varepsilon$

$\nabla \nabla 0^{\circ} \angle L$

09E $\angle L$

$9 \angle 9 \angle L$

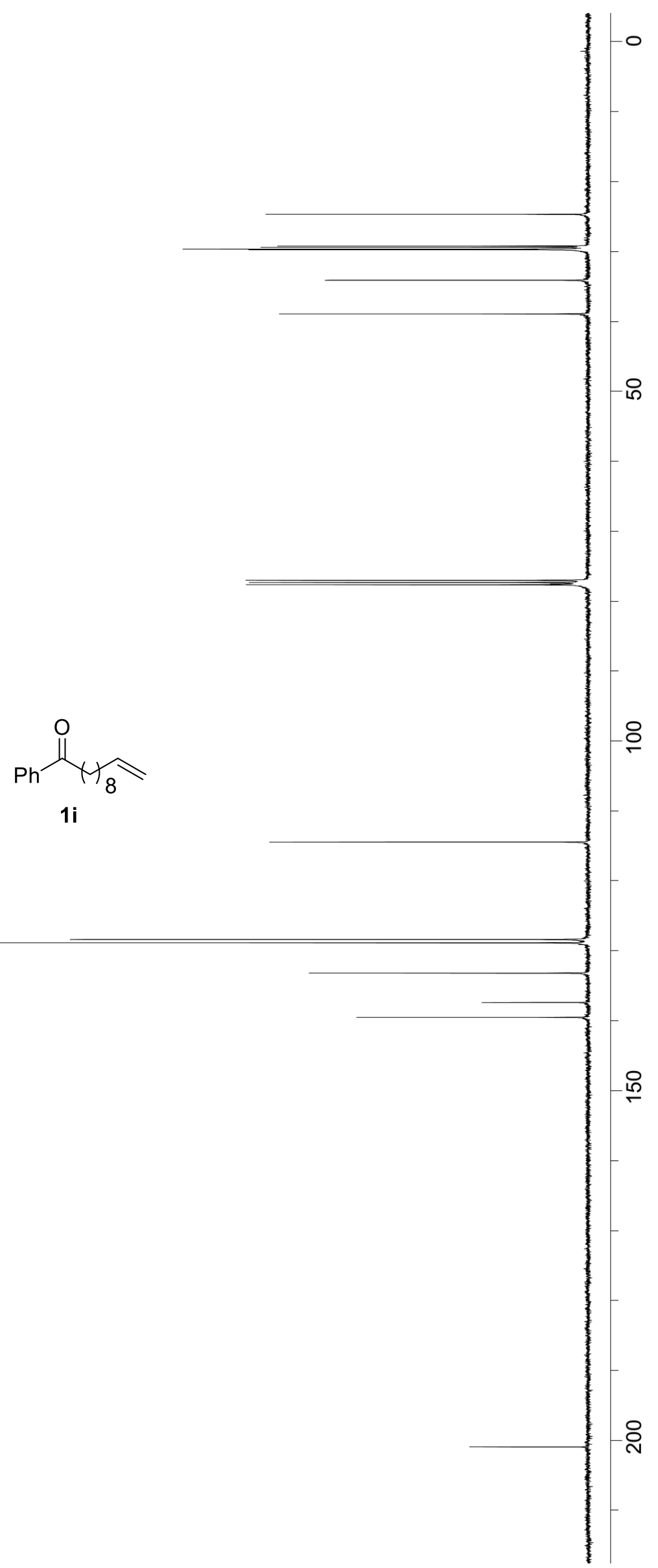

698.82

$06 L^{\circ} \varepsilon \varepsilon \downarrow$

$\varepsilon 6 \varepsilon^{\prime} \angle \varepsilon L$

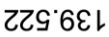



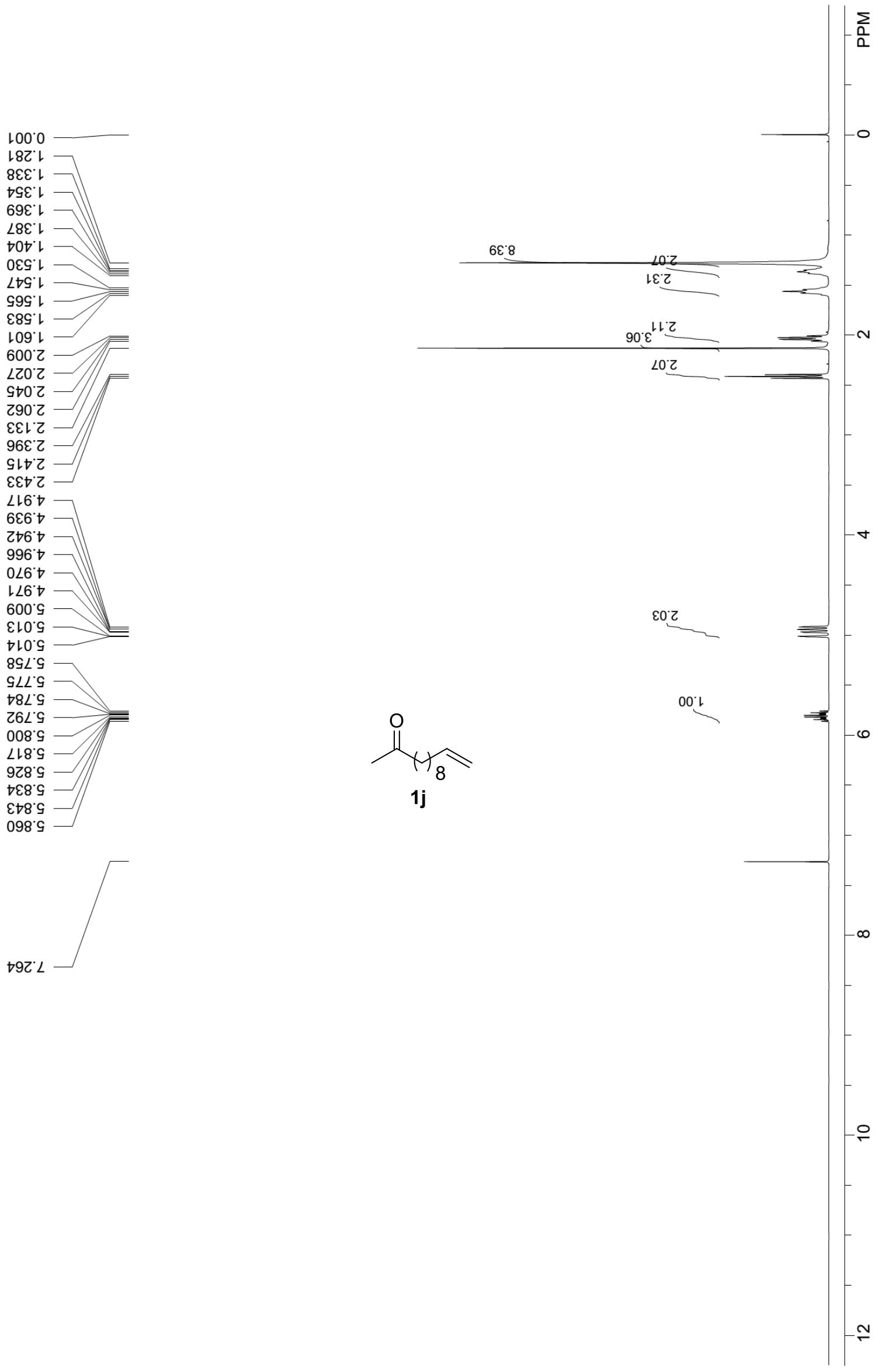

S-38 

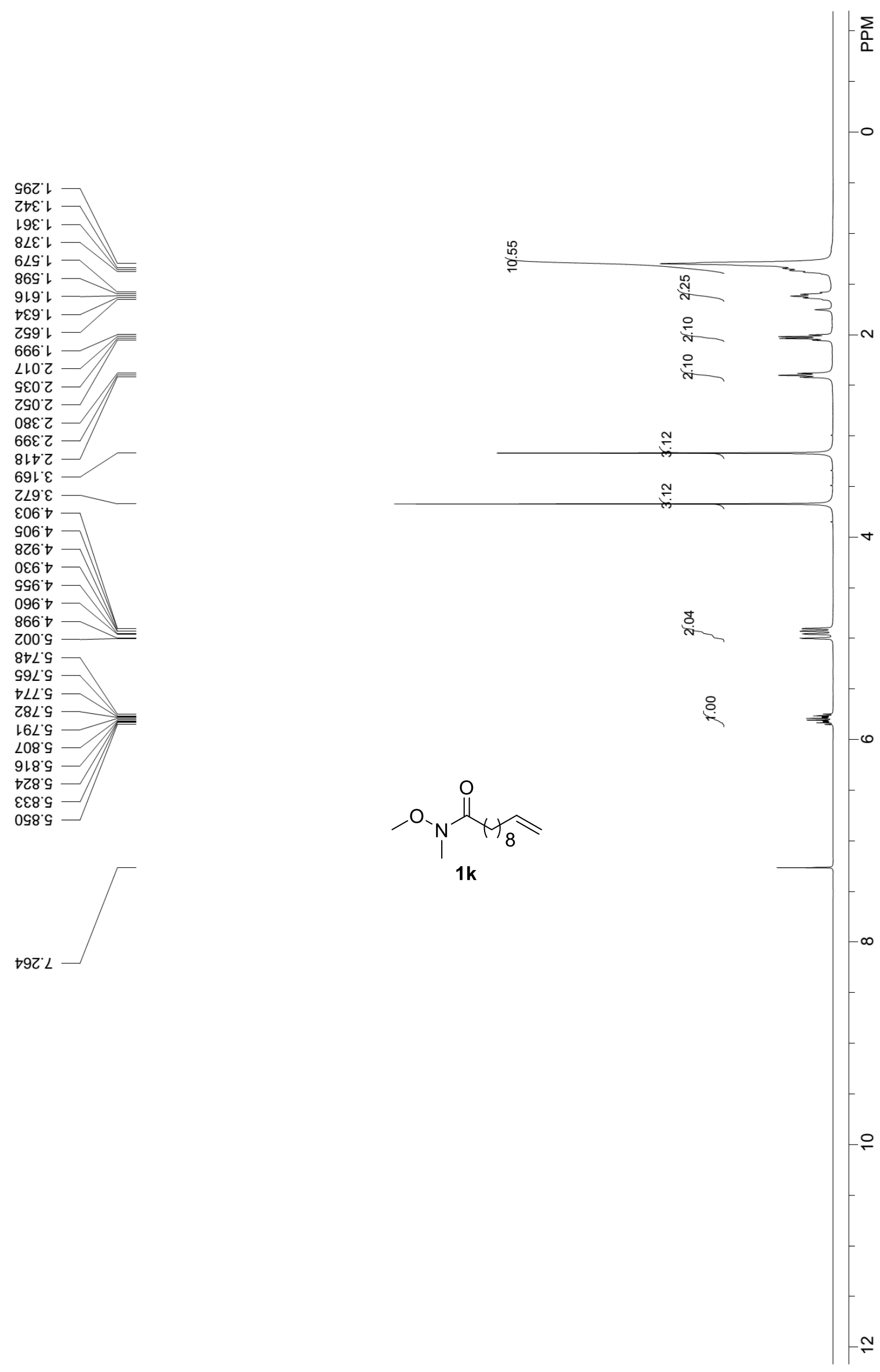

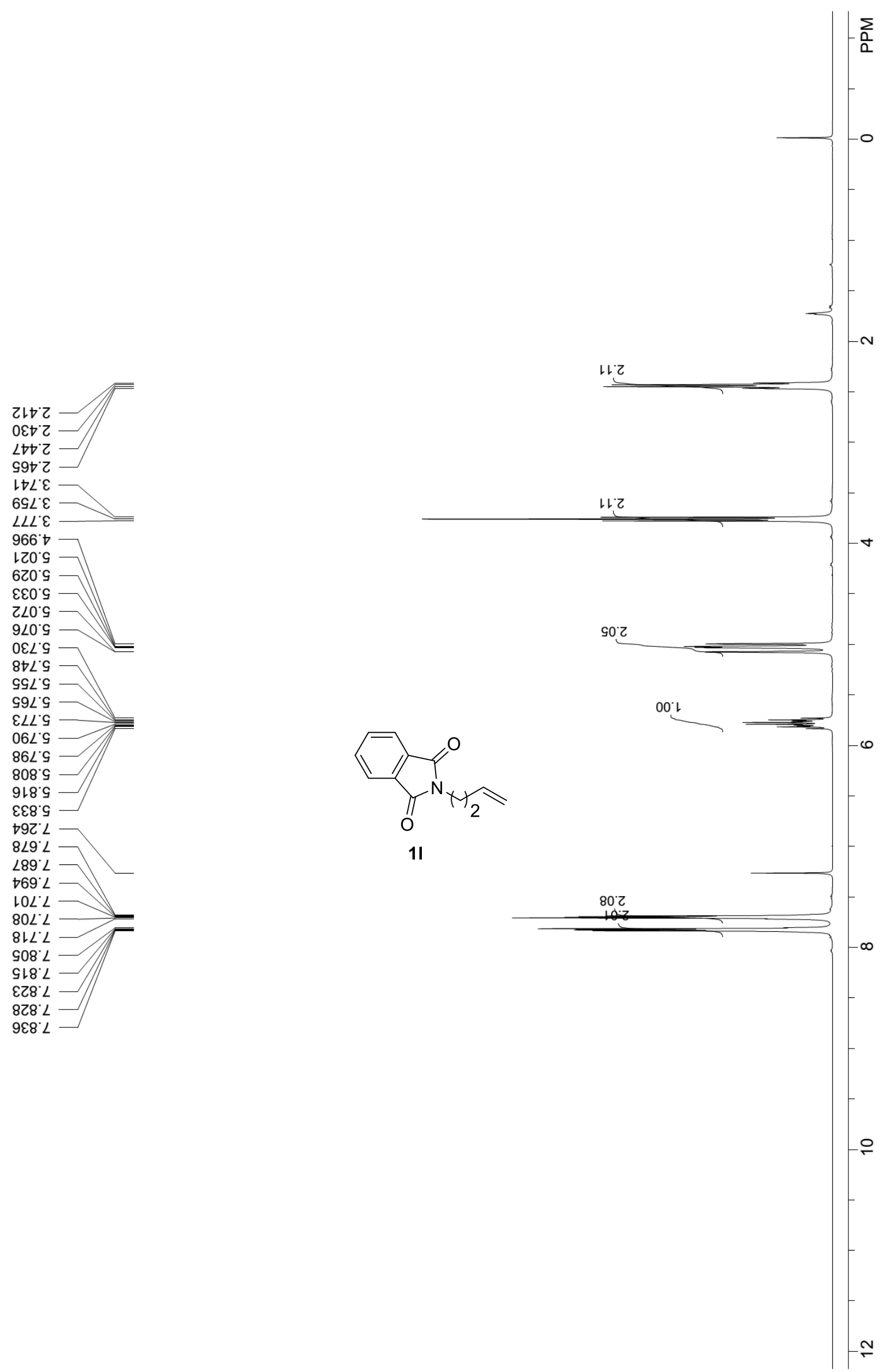

S-40 

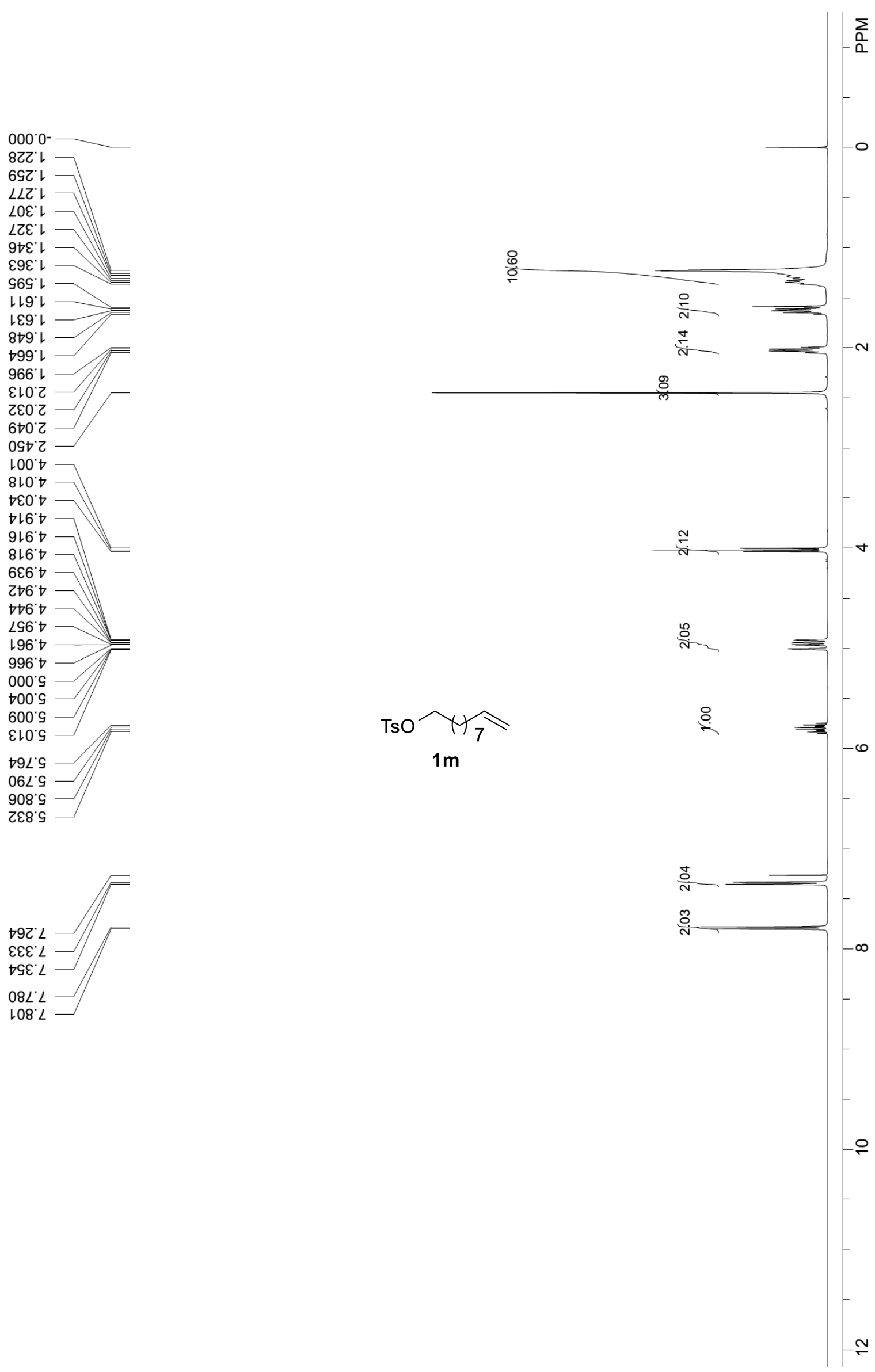

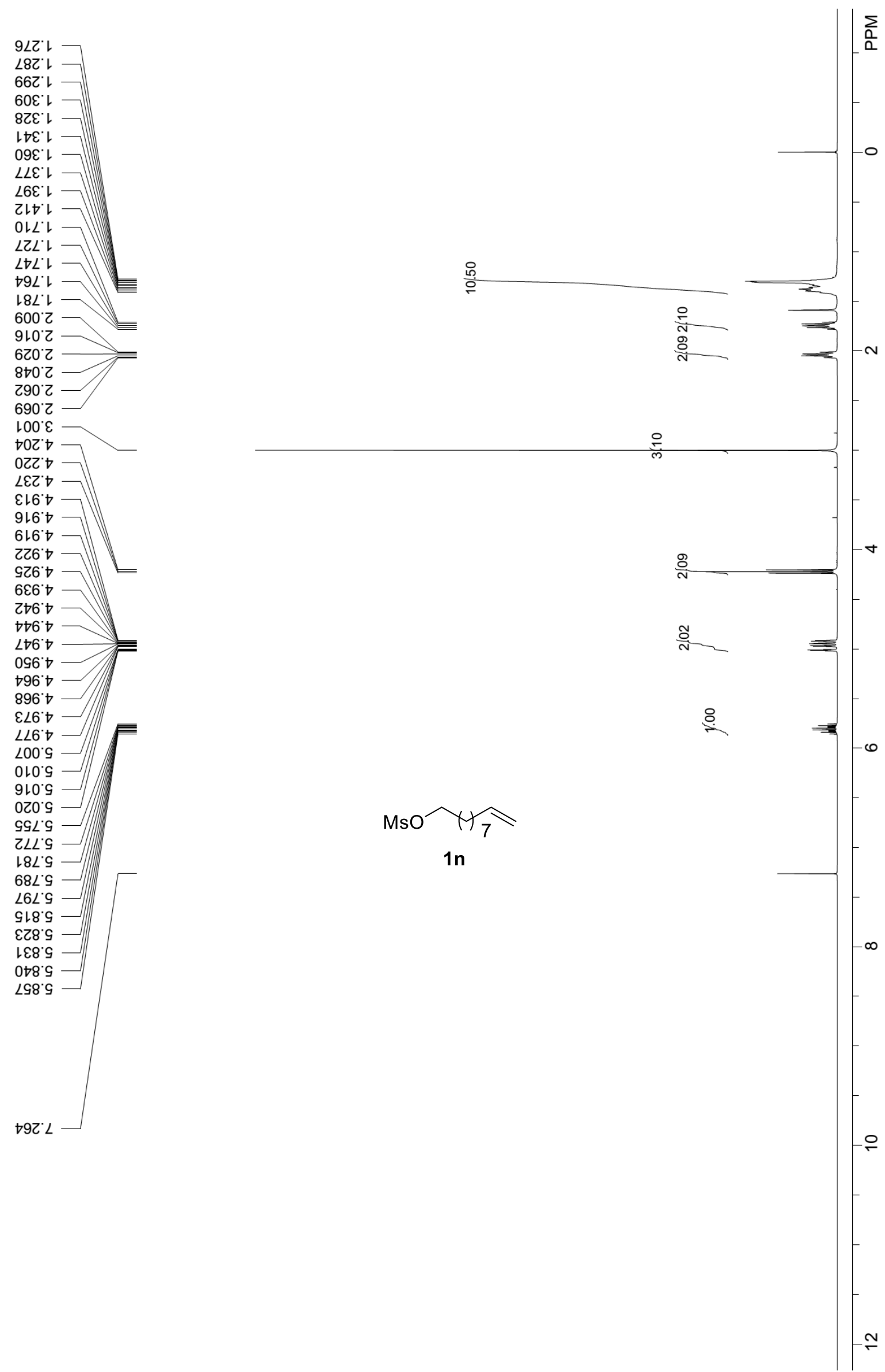


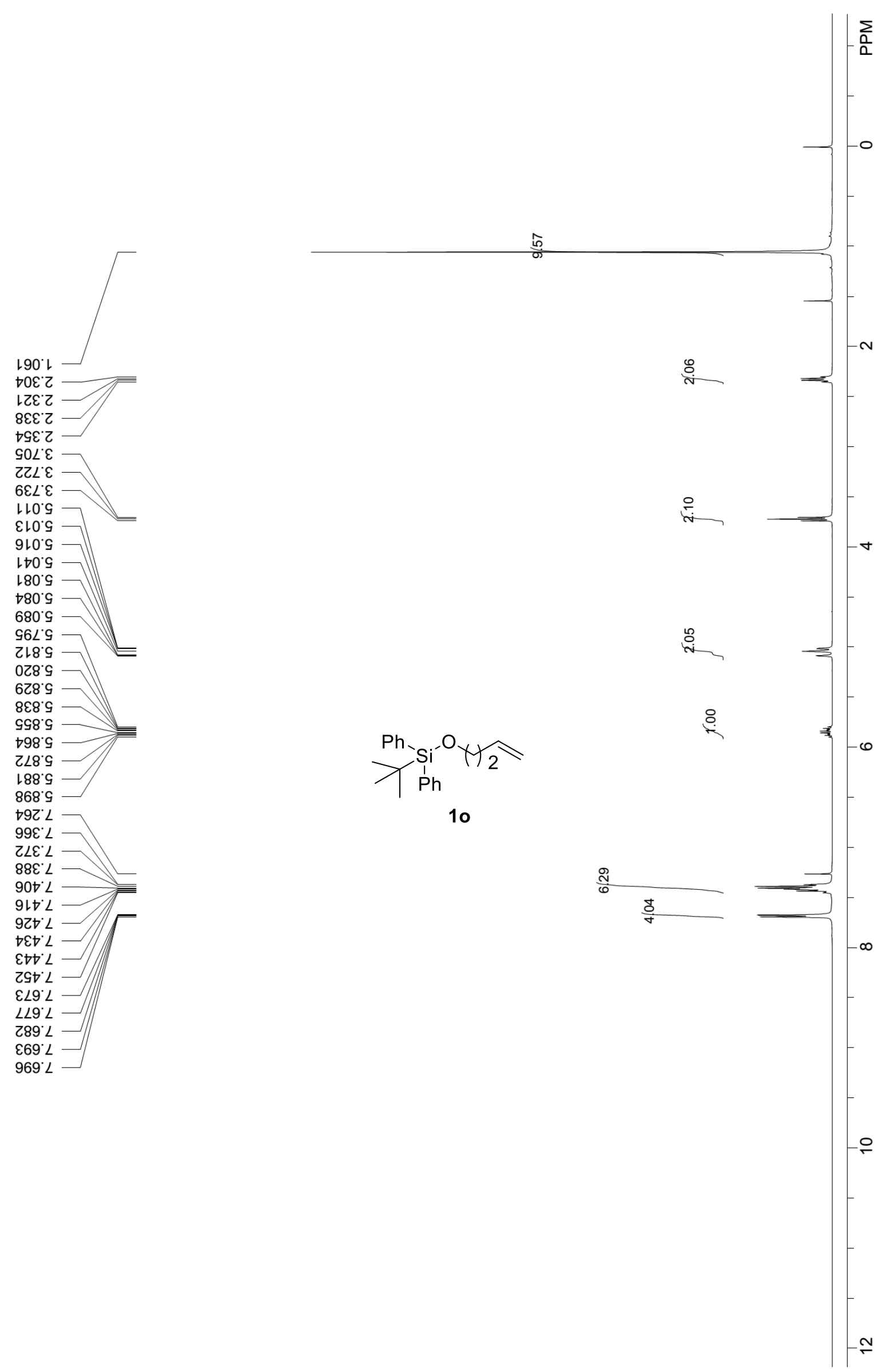



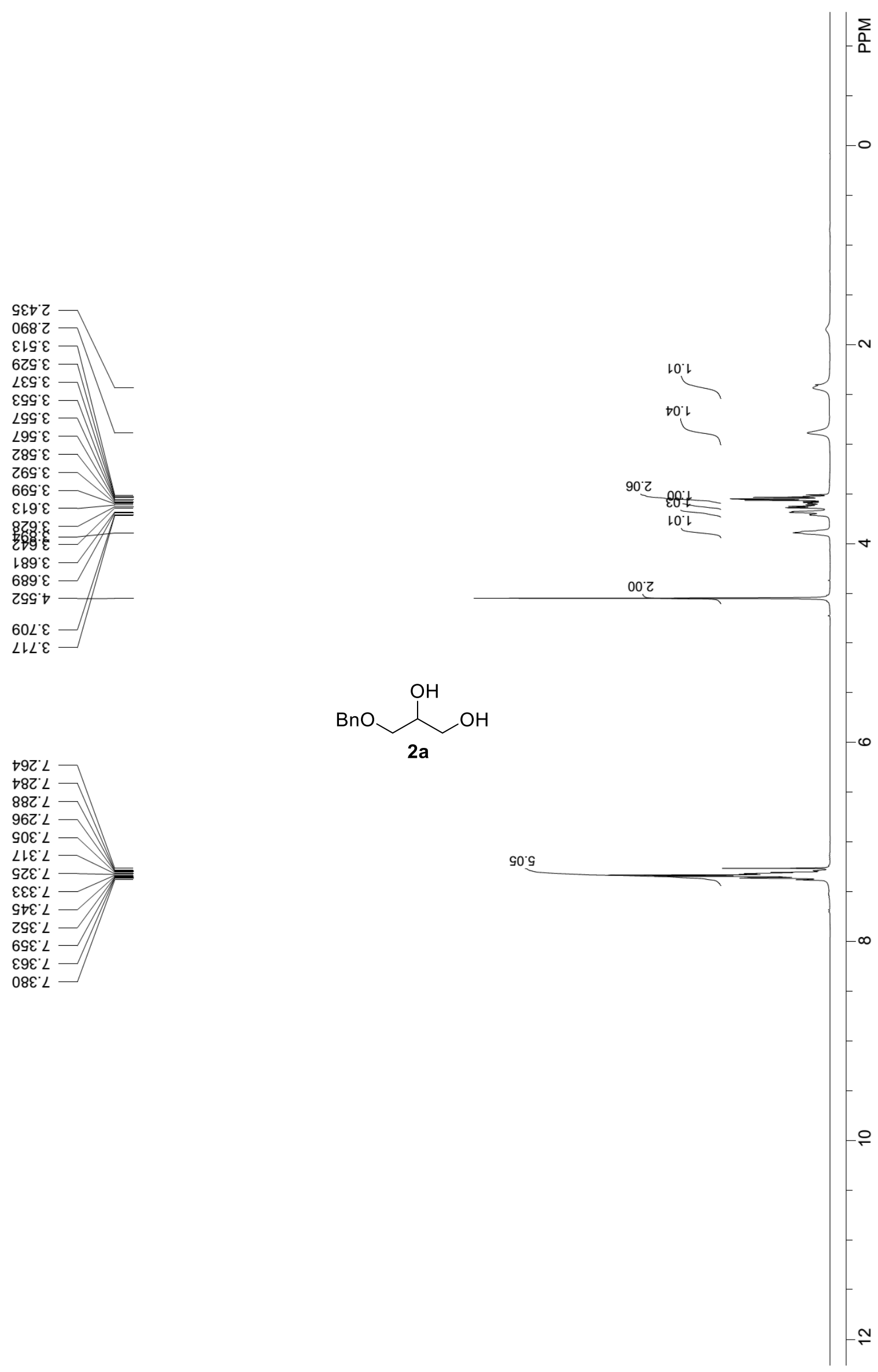

$9 \mathrm{C}^{\circ} \mathrm{L}$

$88{ }^{\circ} L$

$962 \mathrm{~L}$

SOE' $L$

LIE'L

GZE'L

$\varepsilon \varepsilon \varepsilon^{\circ} L$

G†E'L

ZSE'L

$69 \varepsilon^{\circ} L$

ह9ع 'L

$08 \varepsilon^{\circ} L$ 

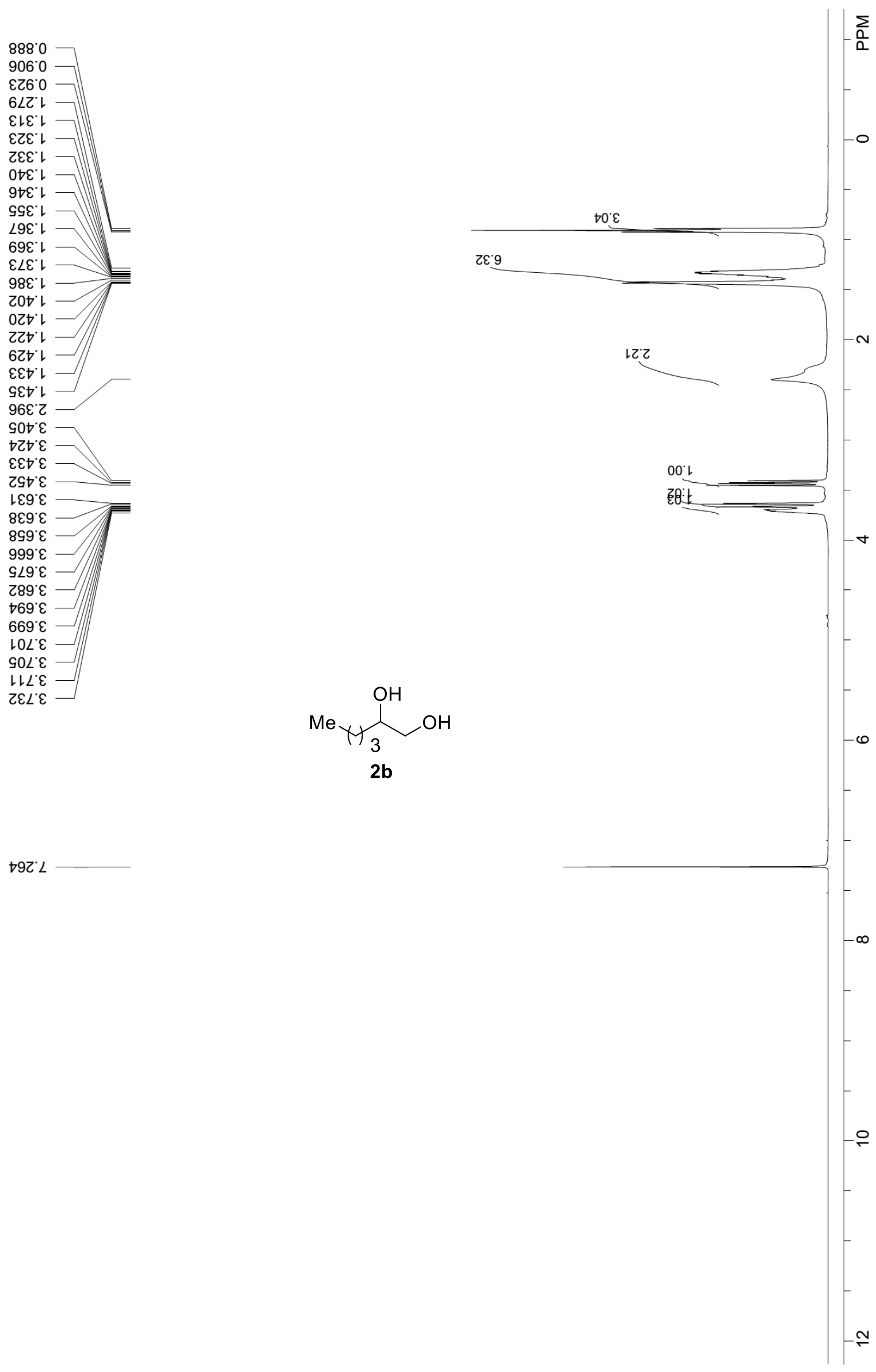

t9Z'L 
$998^{\circ} 0$

ह88.0

$668^{\circ} 0$

OLE'

GSE'L

LレVI

Lてレ \

8E†'

乙†マレ

$\angle \nabla \nabla \cdot$

$\varepsilon 90 \%$

ZSL'Z

乙เt $\varepsilon$

$\nabla \varepsilon \nabla^{\top} \varepsilon$

$8 \varepsilon+\cdot \varepsilon$

6St $\varepsilon$

st9. $\varepsilon$

$\varepsilon \angle 9^{\circ} \varepsilon$

OLL' $\varepsilon$

$O Z L \cdot \varepsilon$

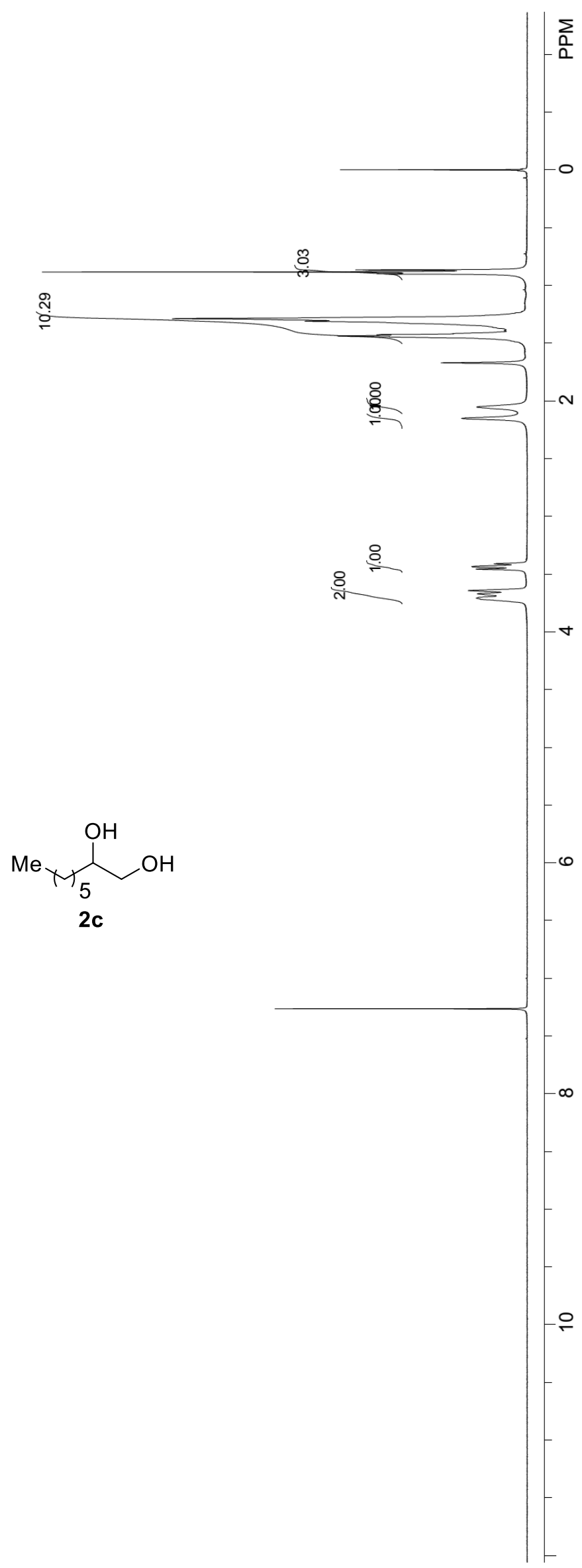

S-46 

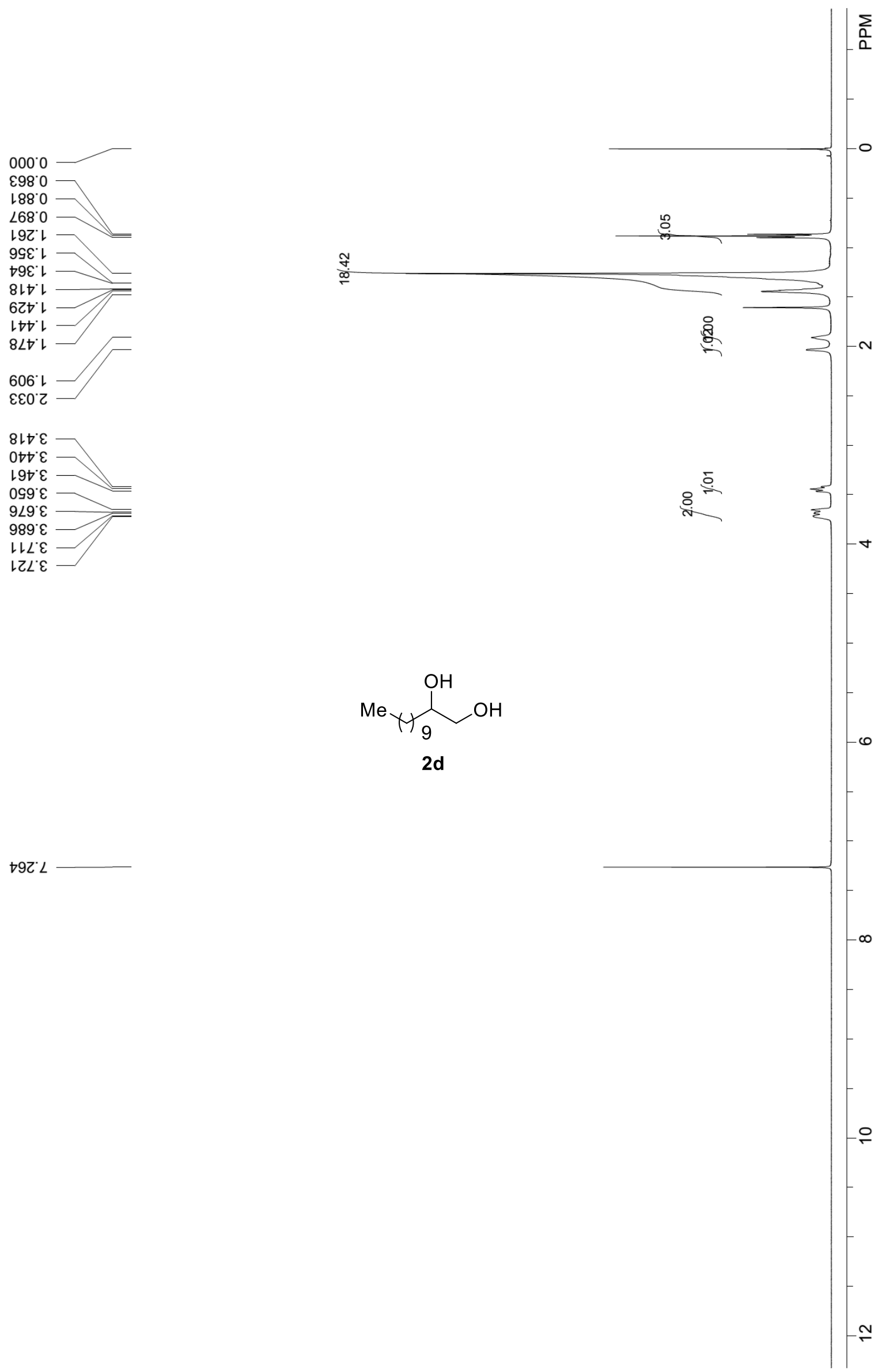

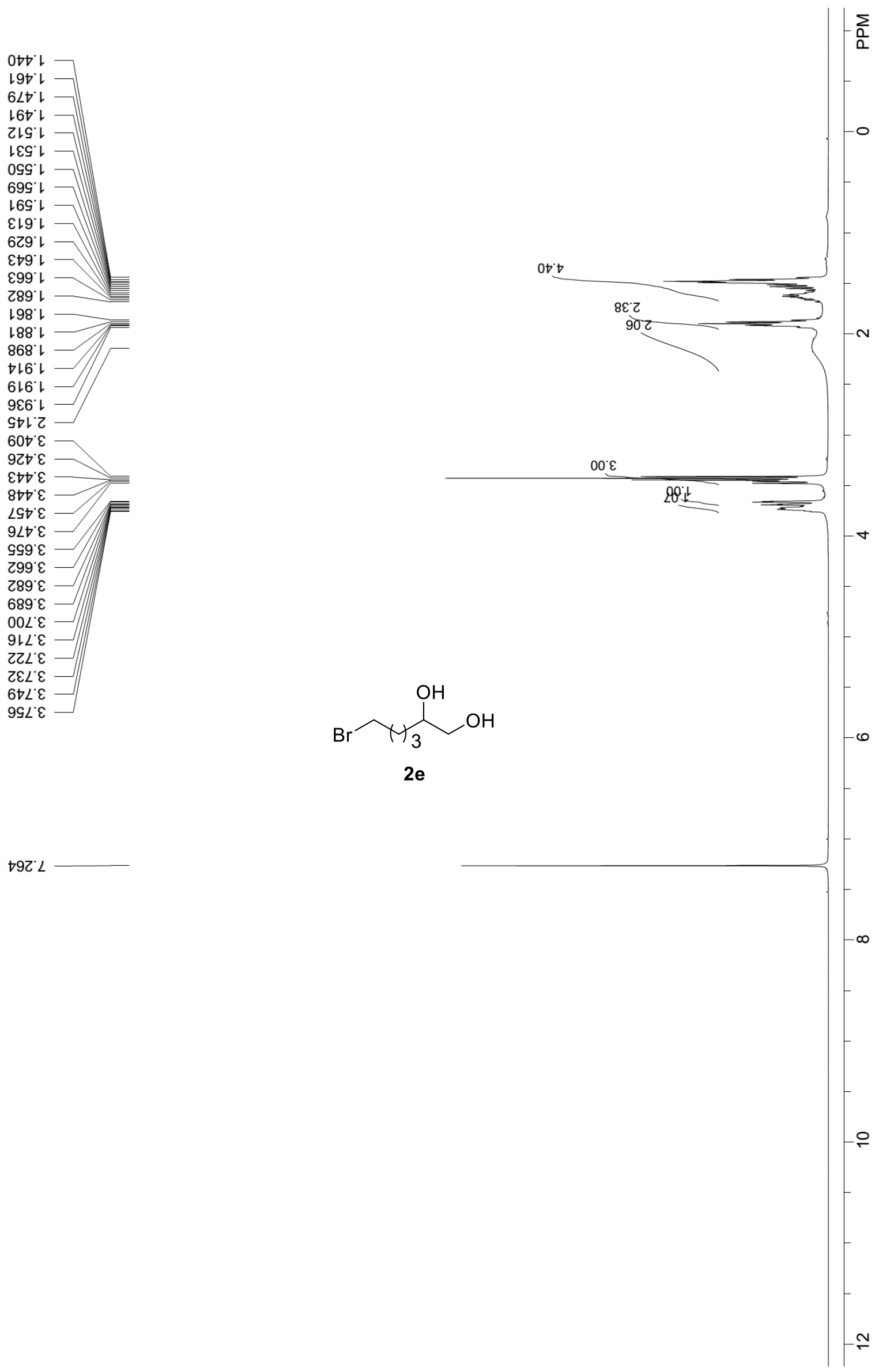

S-48 
เES $\succcurlyeq 乙$

乙8t' $2 \varepsilon$

งะ6'

เท6' $\varepsilon \varepsilon$

$090<9$

$90 \varepsilon^{\prime} Z L$

$\varepsilon \forall 0 \angle L$

$09 \varepsilon^{\prime} L L$

${ }_{9 \angle 9}^{0} \angle L<-\square$

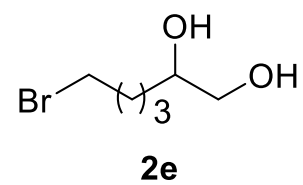

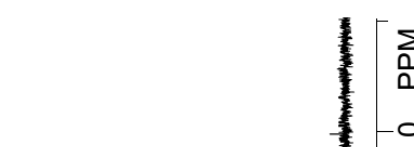

$\frac{\sum_{0}}{0}$

요

음

욤

-으

S-49 

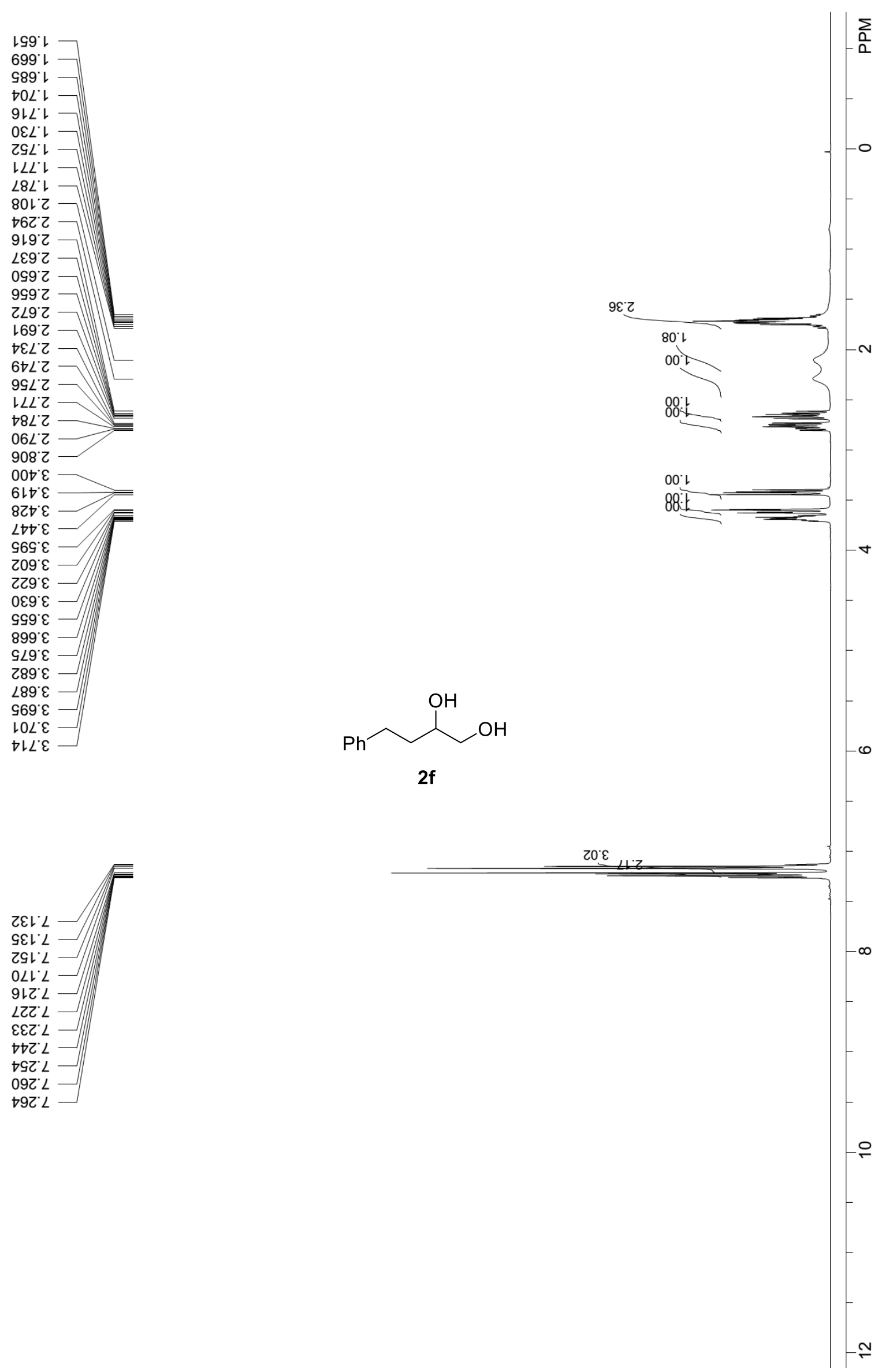

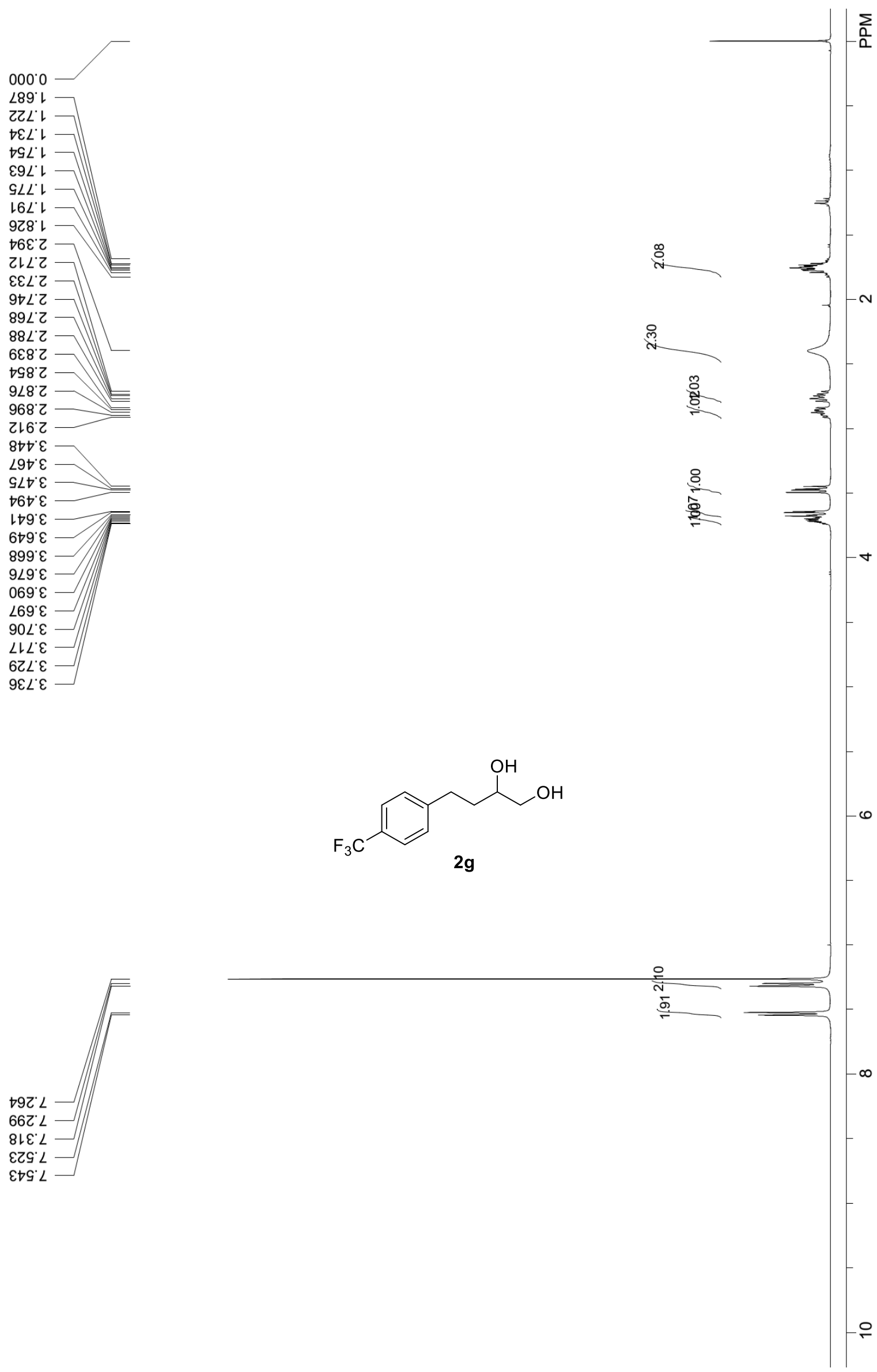

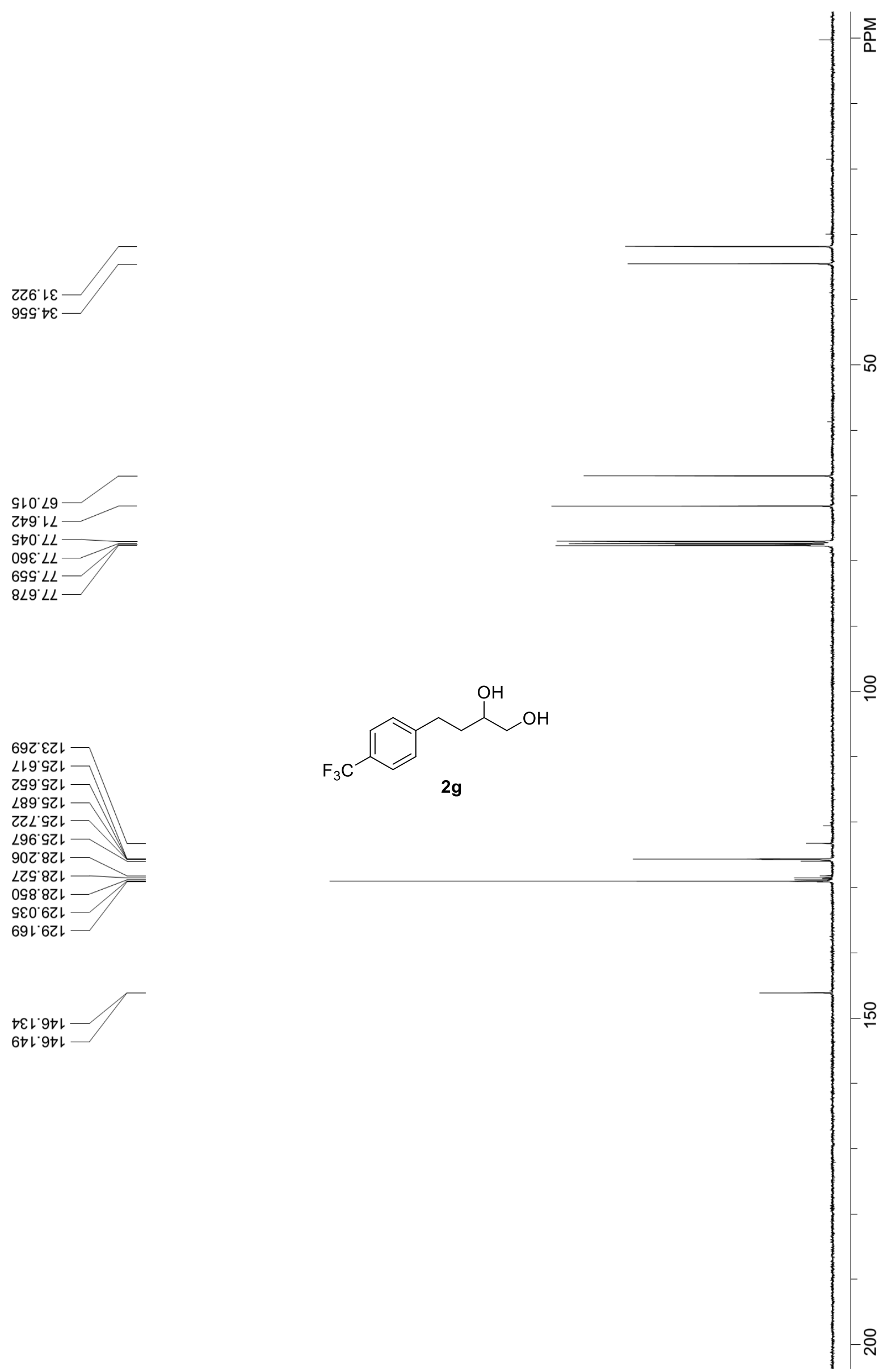


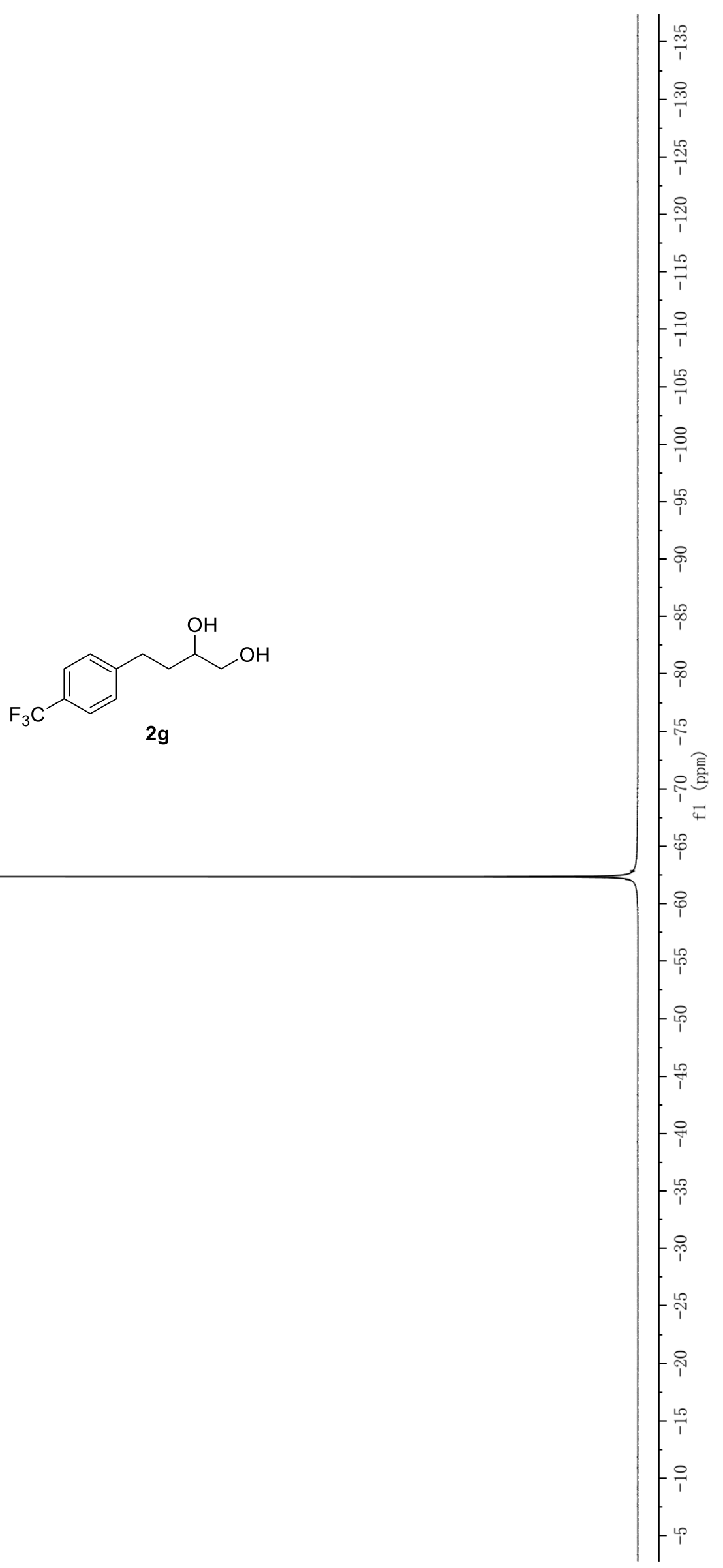

S-53 

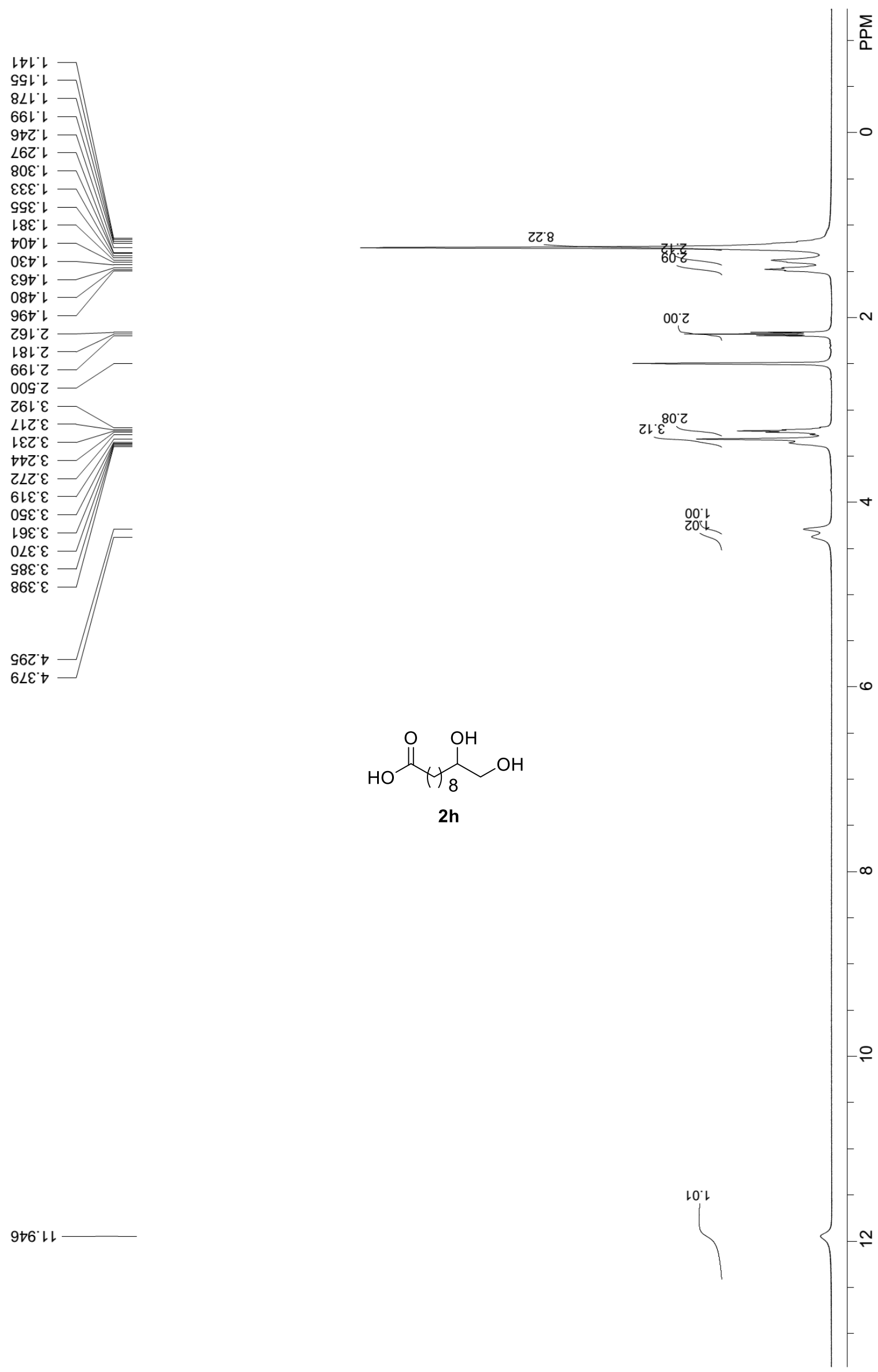

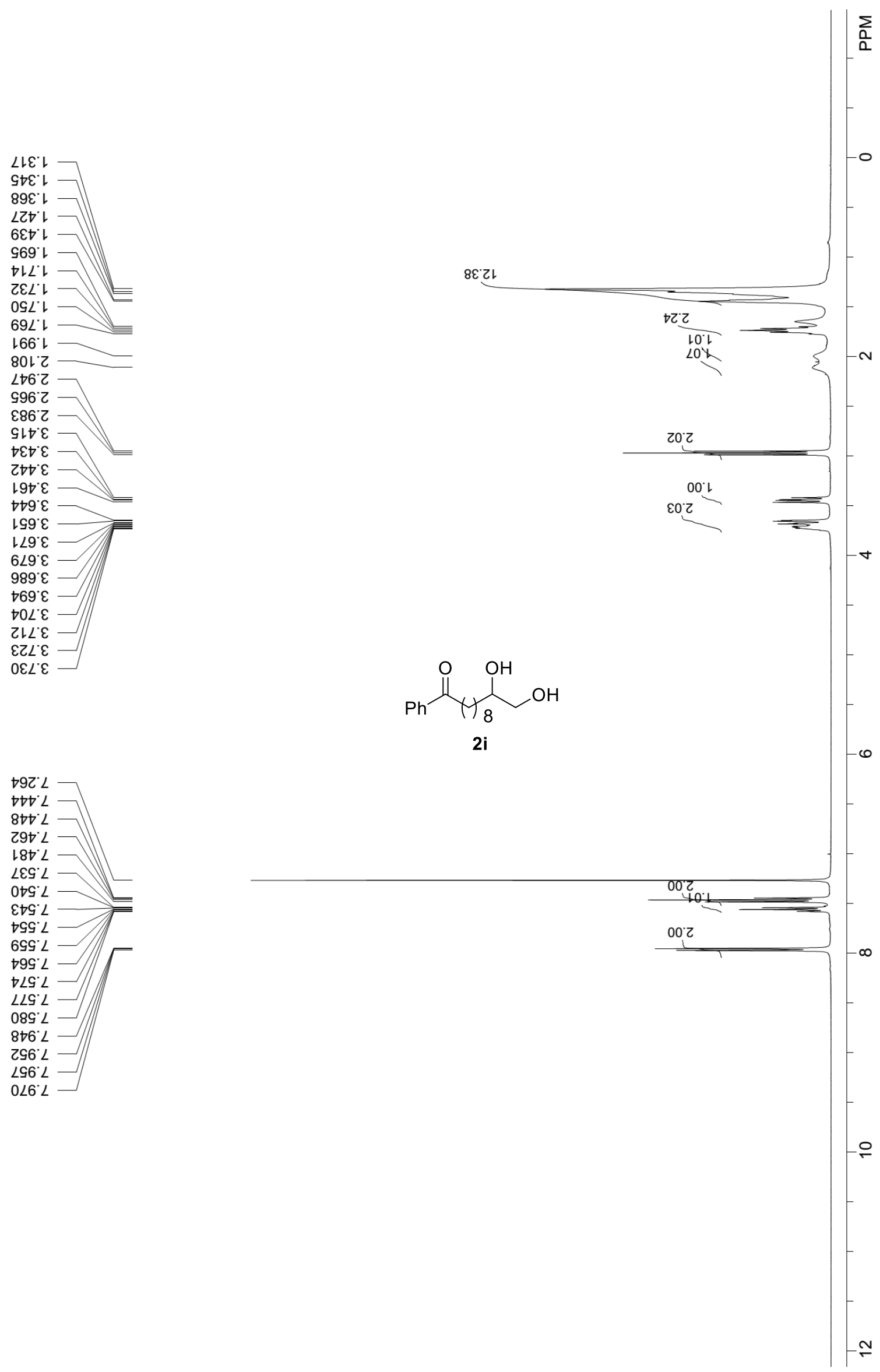


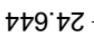

乙เ8

$0 \varepsilon 9^{\circ} 6 Z$

†99.6Z

$989^{\circ} 6 \mathrm{Z}$

$698^{\circ} 62$

$66 t^{\circ} \varepsilon \varepsilon$

५ร6 $8 \varepsilon$

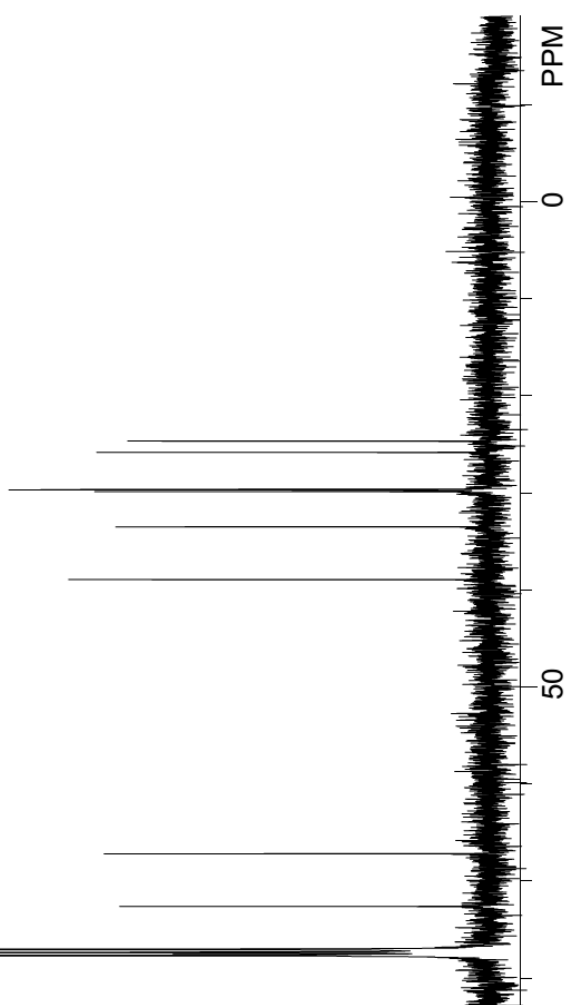

$\angle 6 L^{\circ} \angle 9$

GZ9'ZL

$870 \circ L$

09:L

$789^{\circ} \mathrm{LL}$<smiles>O=C(NC(O)CO)c1ccccc1</smiles>

0 t.'8ZL

†เ6.8ZL

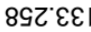

SOt $\angle \varepsilon L$

$9 \varepsilon 0.10 Z$ 
$98 Z$ '

6马E'

$\varepsilon \varepsilon \nabla^{\circ}$

Z†G

6S9'

$9 \angle 9^{\prime} L$

S6G'

$896^{\circ} \mathrm{L}$

$\varepsilon 60^{\circ} \mathrm{Z}$

9عL'ح

00ヤ乙

8เたて

LEt $Z$

てレナと

ธع७ $\varepsilon$

$\angle \nabla^{\circ} \varepsilon$

$9 \succ 9^{\circ} \varepsilon$

$\nabla \angle 9^{\circ} \varepsilon$

$089^{\circ} \varepsilon$

$80{ }^{\circ} \mathcal{E}$

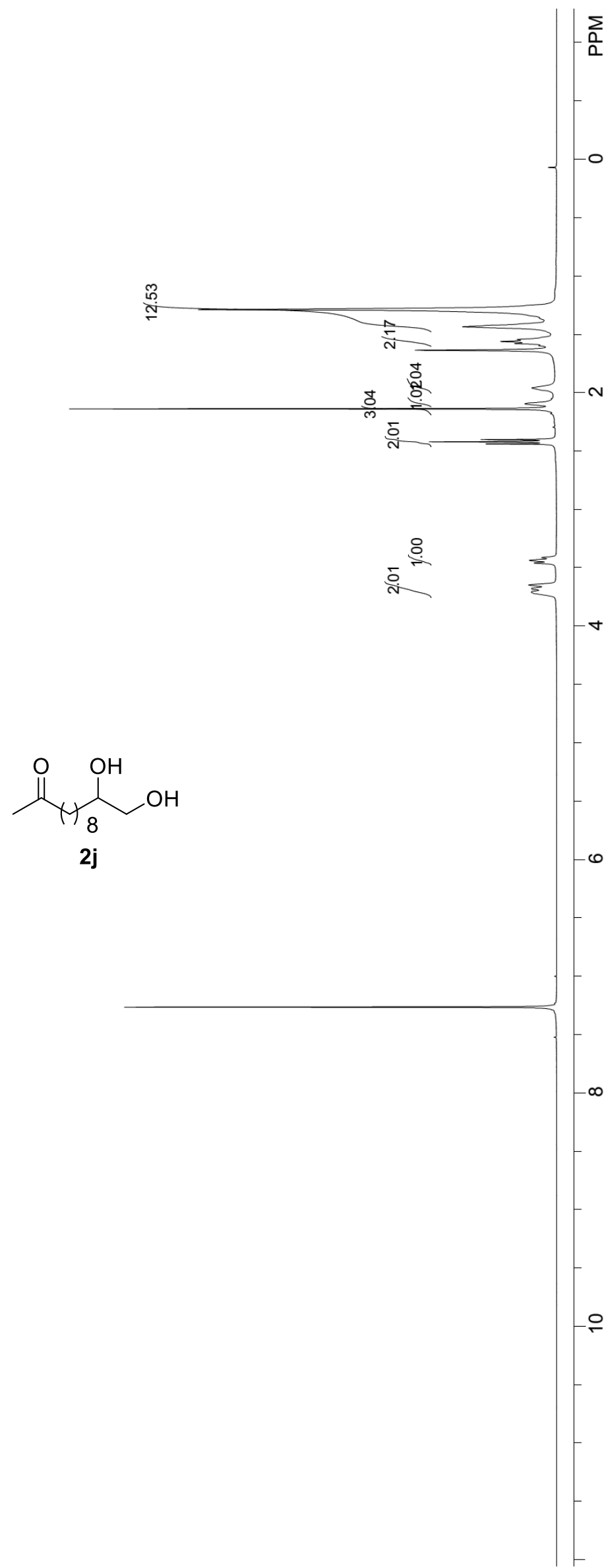

S-57 


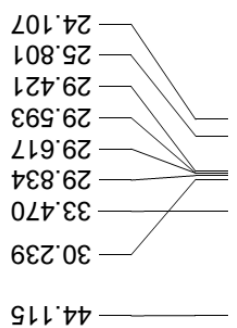

$881: \angle 9$

乙09'乙L

17022

Lt

$\angle \angle 9^{\circ} \angle L$

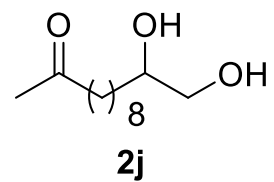

606.602

S-58 

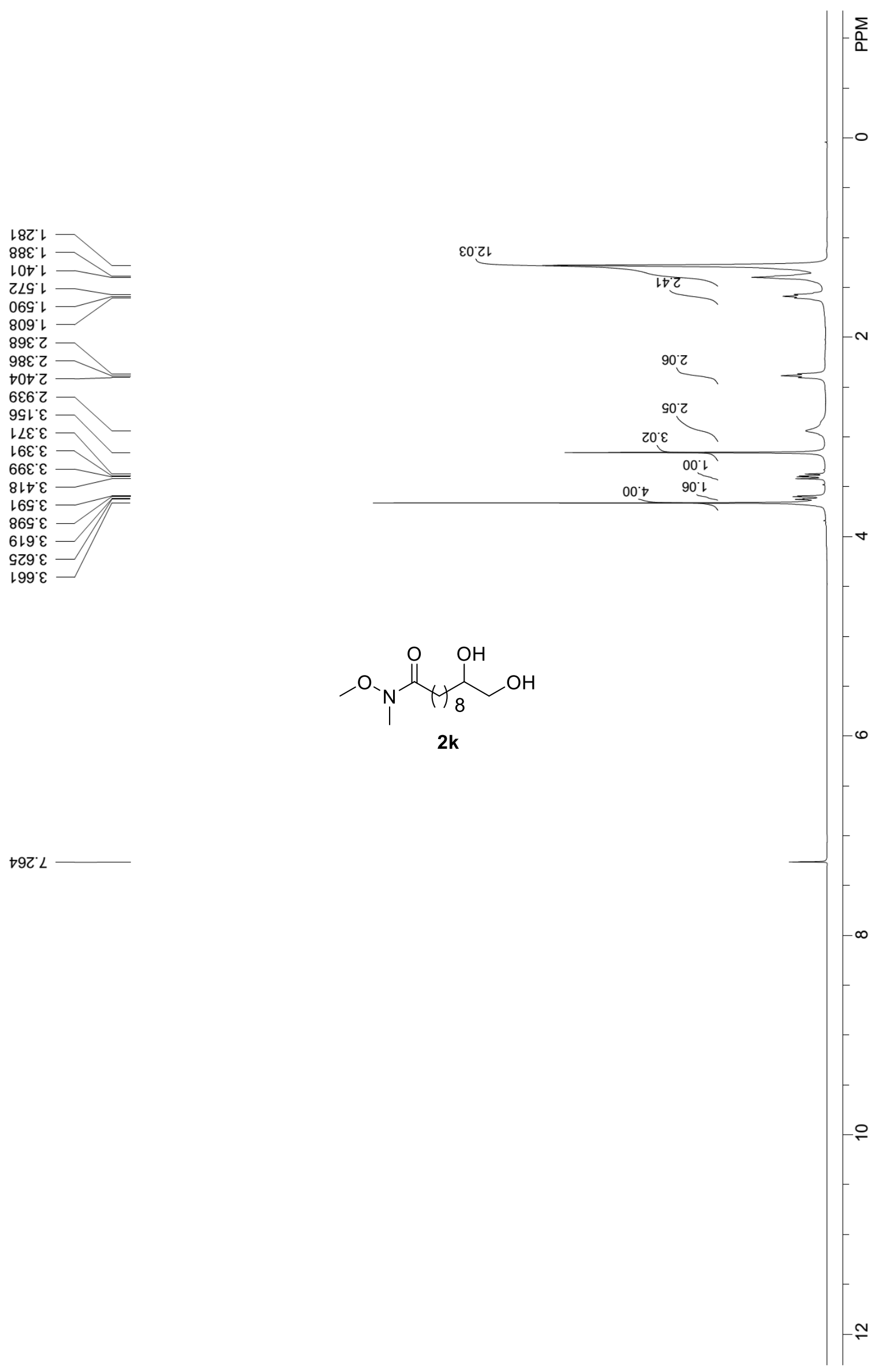


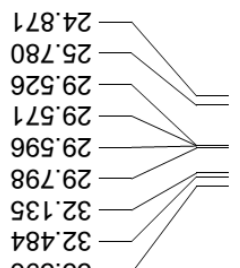

$96 \varepsilon^{\circ} \varepsilon \varepsilon$

98t' 19

$11 L: 29$

Z9G'ZL

$6 \varepsilon 0^{\circ} \angle L$

$09 \varepsilon^{\prime} \angle L$

L $\angle 9^{\circ} \angle L$

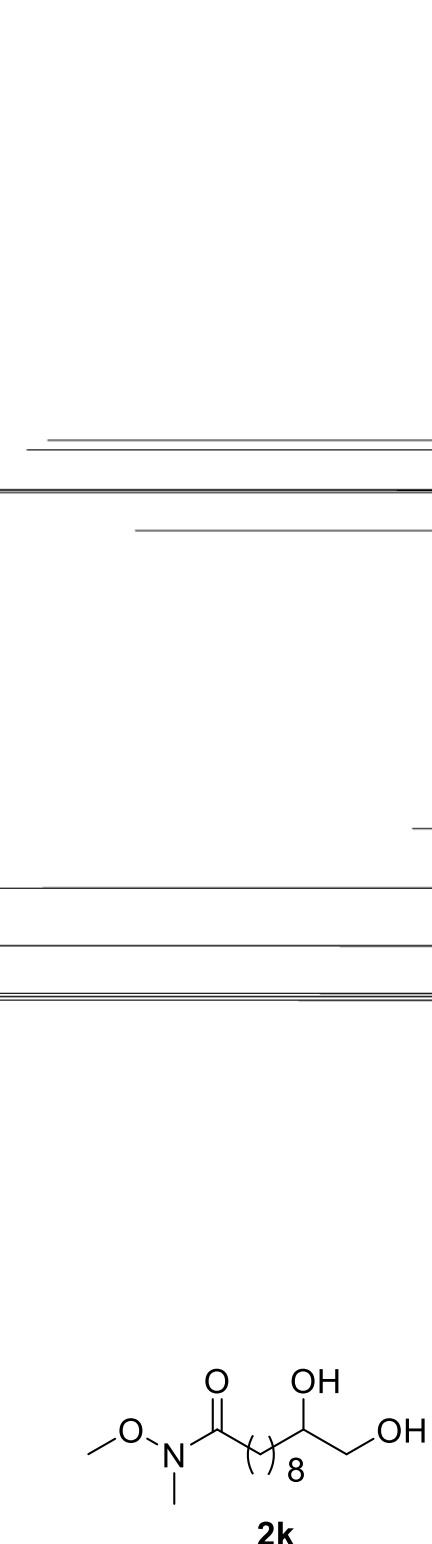

2k

Z৪L'GLL 

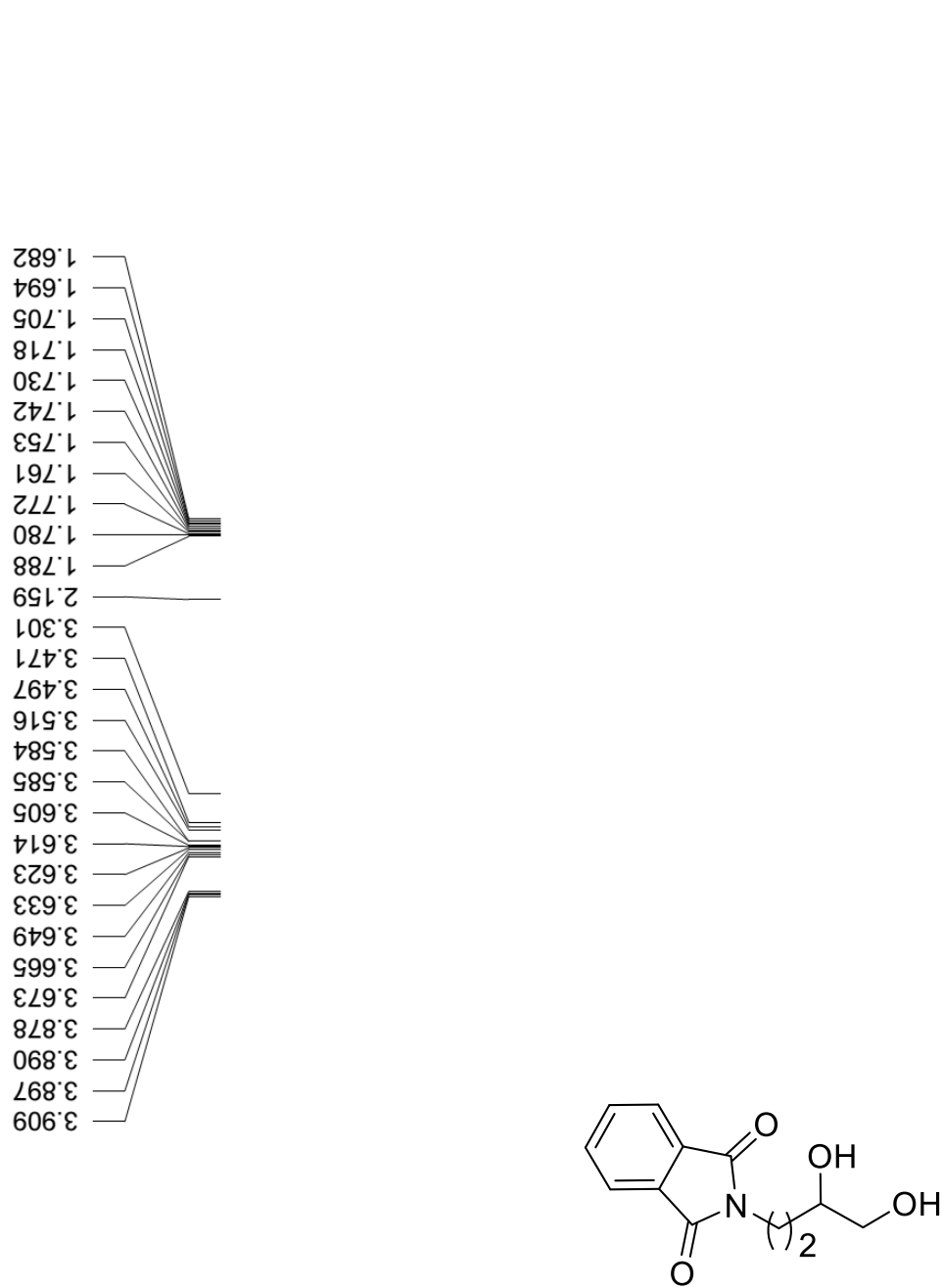

||$\sum-\frac{2}{0}$

$\sum_{0}$

2I

๑9Z: L

$8 Z L^{\prime} L$

$9 \varepsilon L L$

$Z \nabla L L L$

$6 \nabla L L$

$09 L L$

$8 \varepsilon 8$ ' $L$

$\angle \neg 8^{\circ} L$

$998^{\circ} \mathrm{L}$

$198^{\circ} \mathrm{L}$

$698^{\circ} L$

$8 \angle 8^{\circ} \mathrm{L}$
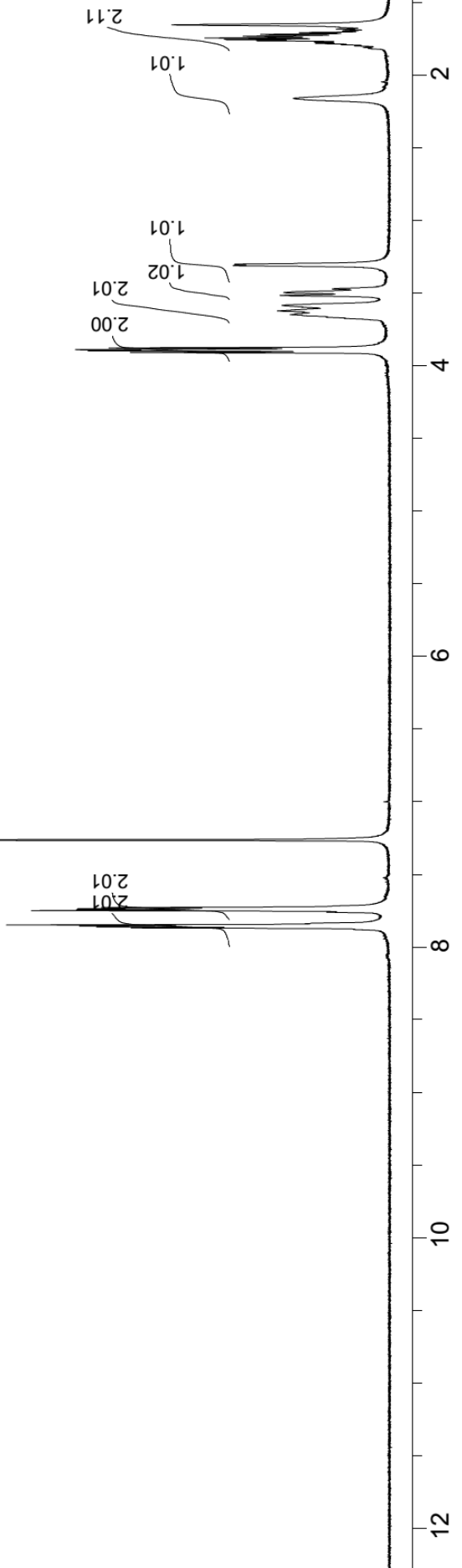
乙L乙'乙E

$6 \triangleright L^{\prime} \downarrow \varepsilon$

6乙L'99

GZS'69

$9 \varepsilon 0^{\circ} L L$

$09 \varepsilon^{\prime} \angle L$

$899^{\circ} L$

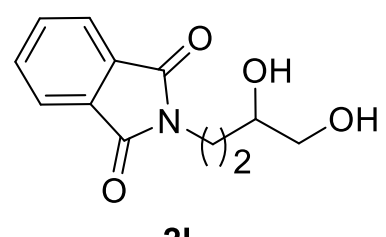

21

$\angle 0 L ' \varepsilon Z L$

LGZ'ZEL

$\varepsilon \angle t^{*} \downarrow \varepsilon L$

$9 l Z^{\prime} 69 l$ 


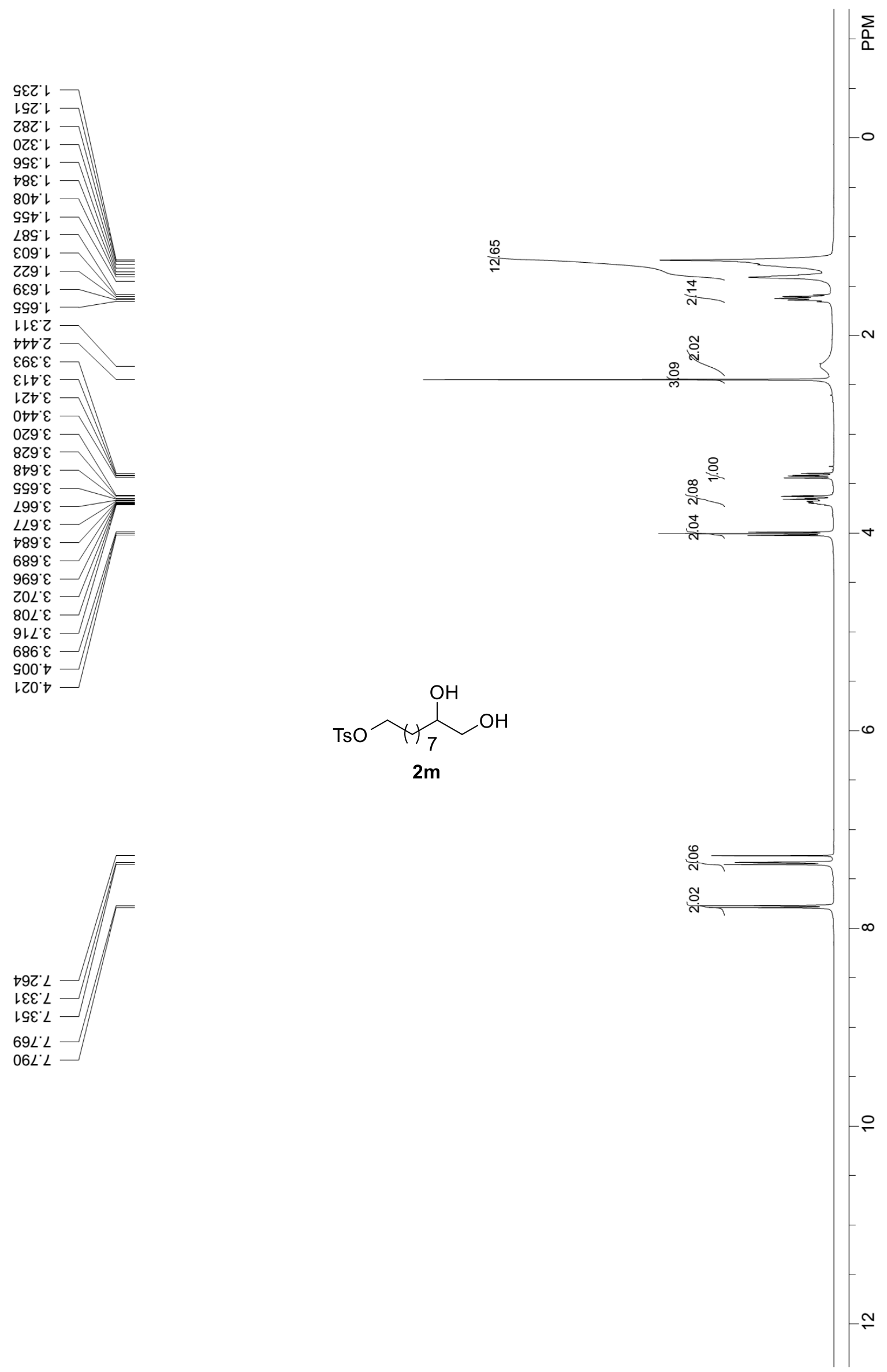


896'ル

069' $9 Z$

EฑL'G乙

260 62

tZ9.6Z

$8 \mathrm{~L} \cdot 6 Z$

$\dashv \varepsilon \nabla^{\circ} \varepsilon \varepsilon$

E9L 29

乙LO'L

$\nabla \angle G^{\prime} Z L$

$\varepsilon \supset 0^{\circ} L L$

$09 \varepsilon^{\prime} L L$

$8 \angle 9^{\circ} L L$<smiles>OCC(O)[Al]CO[As]</smiles>

$2 m$

เLZ'8ZL

StL.0EL

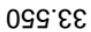

$866^{\circ}$ tヤ

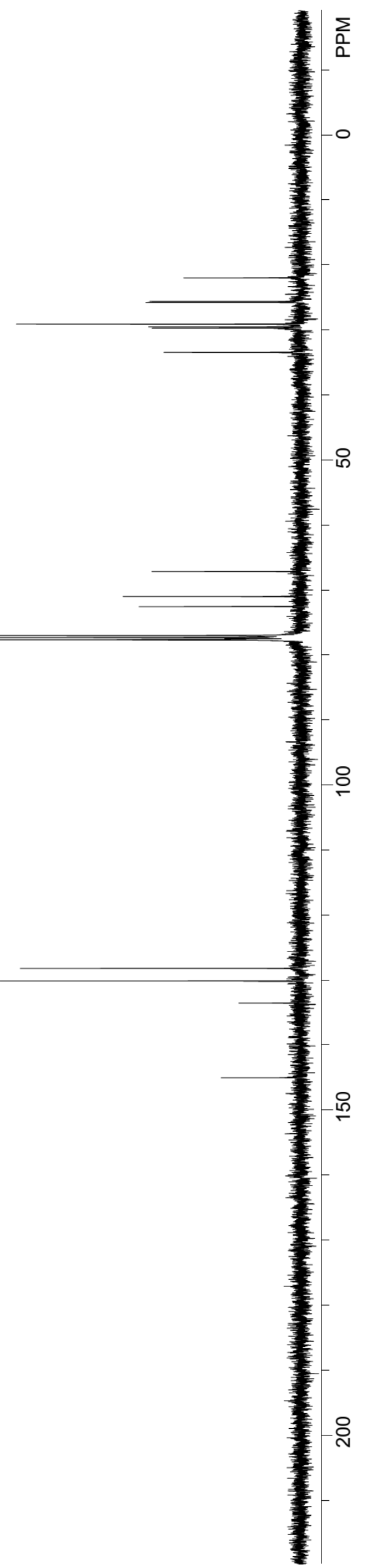


$6 เ \varepsilon^{\circ}\llcorner$

Z9E'
$6 \angle E^{\prime} L$

SOt

Zเ゙๋

9Zt

6Et เ

ILLL

$\angle Z L^{\circ} L$

$\angle \nabla L '$

$\rightarrow 9 L L$

$08 L^{\prime} \cdot$

$696^{\circ} \mathrm{L}$

$90 L^{\prime} \mathrm{Z}$

$\angle 00^{\circ} \varepsilon$

$\varepsilon เ \nabla \varepsilon$

乙ย๋ $\varepsilon$

$0 t \nabla^{\circ} \varepsilon$

$6 \mathrm{St} \varepsilon$

Z๑9 $\varepsilon$

$6 \pitchfork 9^{\circ} \varepsilon$

$0 \angle 9^{\circ} \varepsilon$

$\angle \angle 9^{\circ} \varepsilon$

$069^{\circ} \varepsilon$

$10 L^{\circ} \varepsilon$

$80 L^{\prime} \mathcal{E}$

$6 L L^{\prime} \varepsilon$

$6 \varepsilon L \mathcal{L}$

$\angle O Z^{\prime} \triangleright$

๕Zて॰

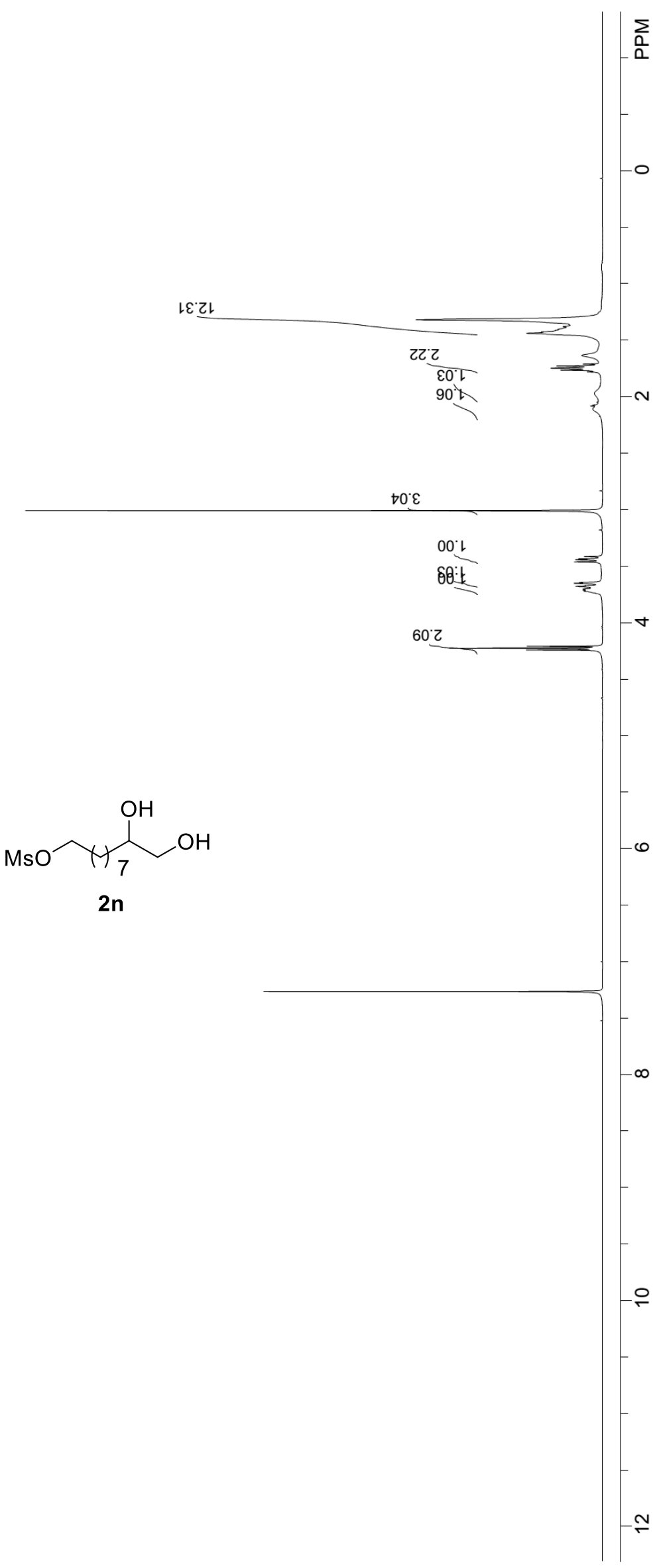

t9Z'L 
699.92

E0t 62

$899^{\circ} 62$

$9 \varepsilon L^{\prime} 6 z$

$92 \downarrow \varepsilon \varepsilon$

$\varepsilon 0 L^{\circ} \angle \varepsilon$

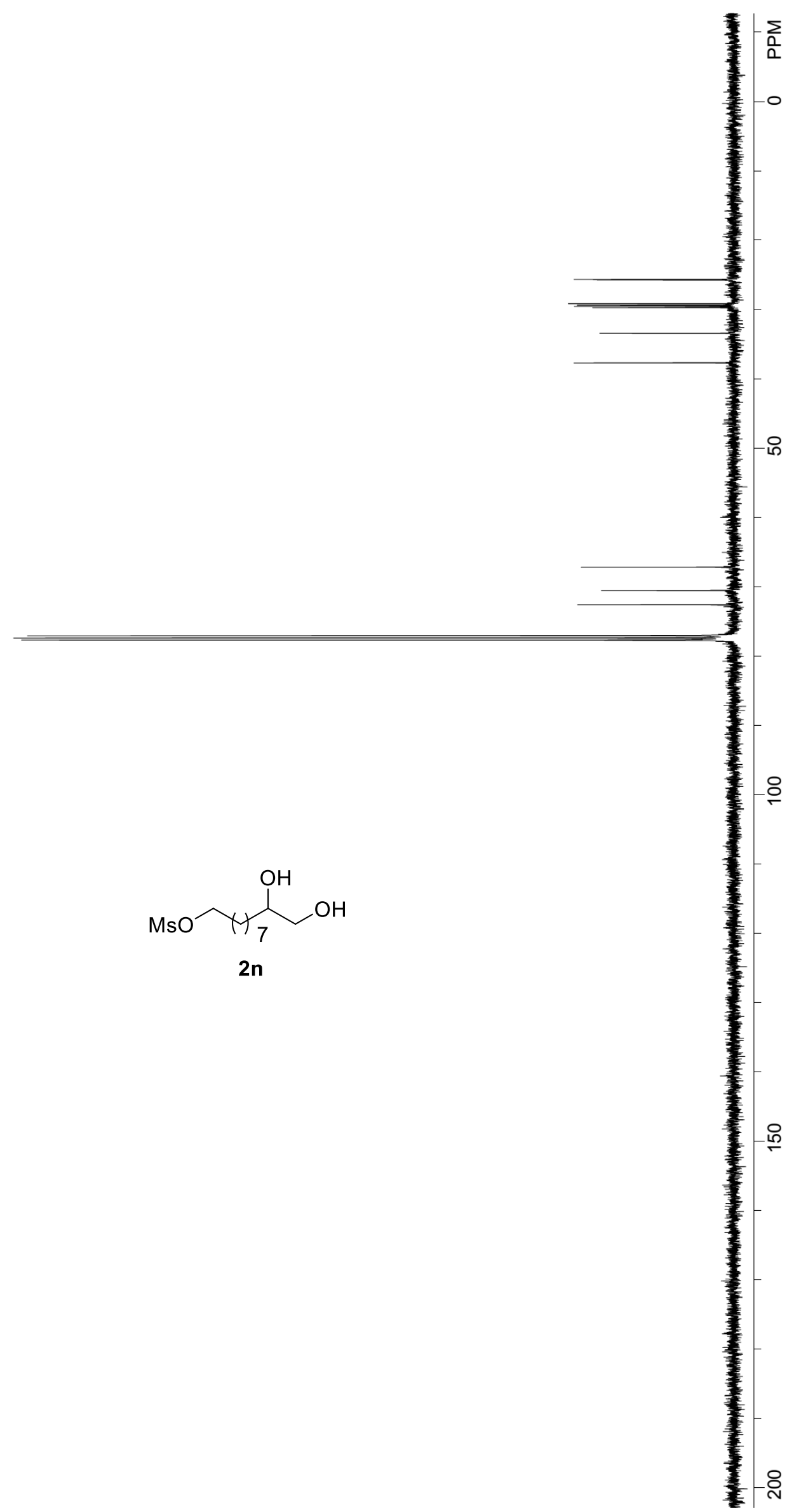

S9l. 29

$\angle 8 S^{\prime} Z L$

$S T 0^{\circ} \angle L$

$09 \varepsilon^{\circ} \angle L$

Z89. 

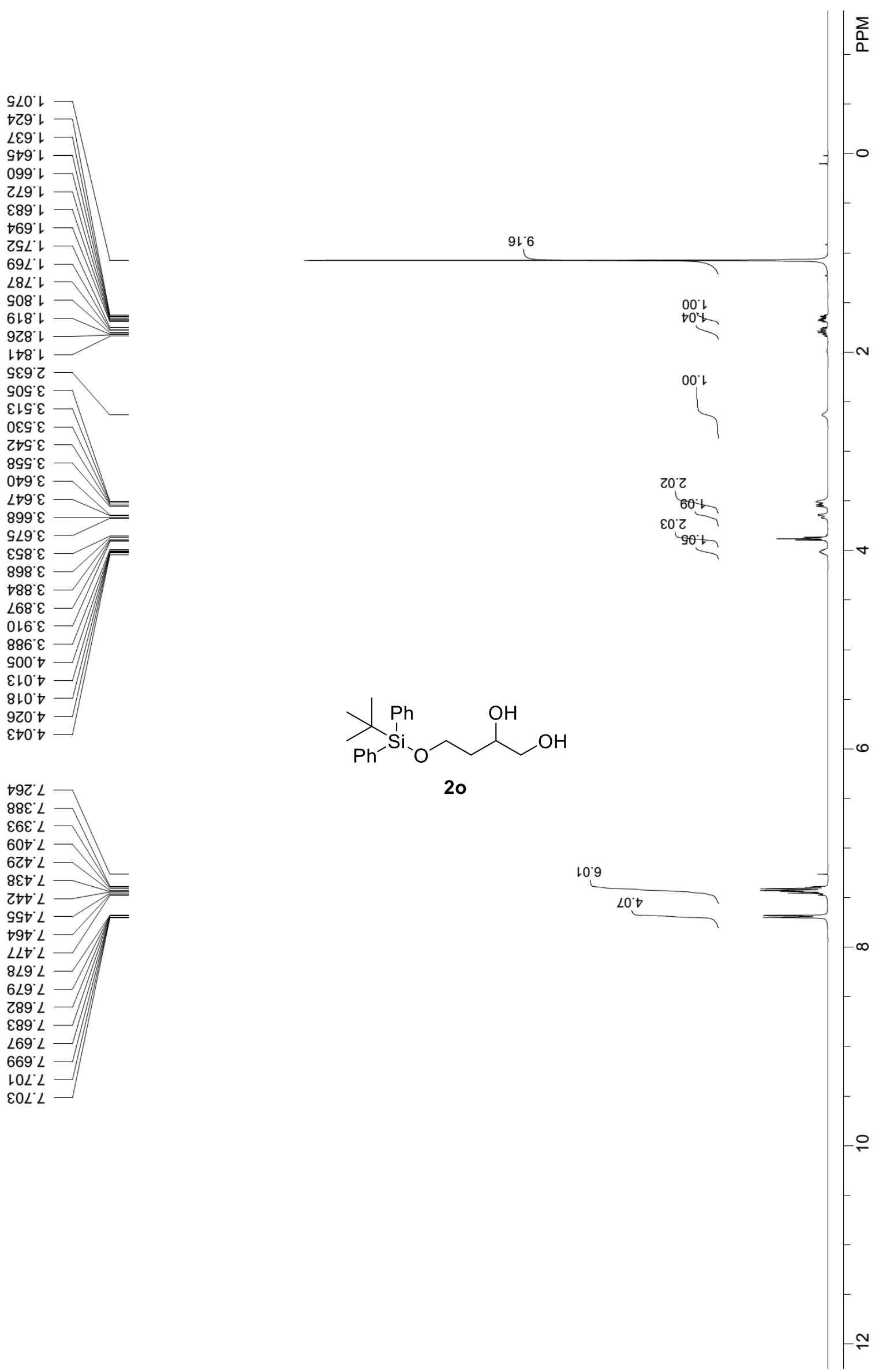

†9ट' L

$88 \varepsilon^{\circ} L$

$\varepsilon 6 \varepsilon^{\circ} L$

$60 t^{\circ} L$

$6 Z \nabla^{\circ} L$

$8 \varepsilon \nabla^{\circ} L$

Ztt L

SSt L

†9t L

$\angle L T \angle$

$6 \angle 9^{\circ} L$

$289^{\circ} \mathrm{L}$

$\varepsilon 89^{\circ} L$

$\angle 69^{\circ} \mathrm{L}$

$669^{\circ} \mathrm{L}$

$10 L ' L$

$\varepsilon 0 L^{\prime} L$ 
عง 6 เ

ELLL乙

$0 เ \downarrow \cdot \varsigma \varepsilon$

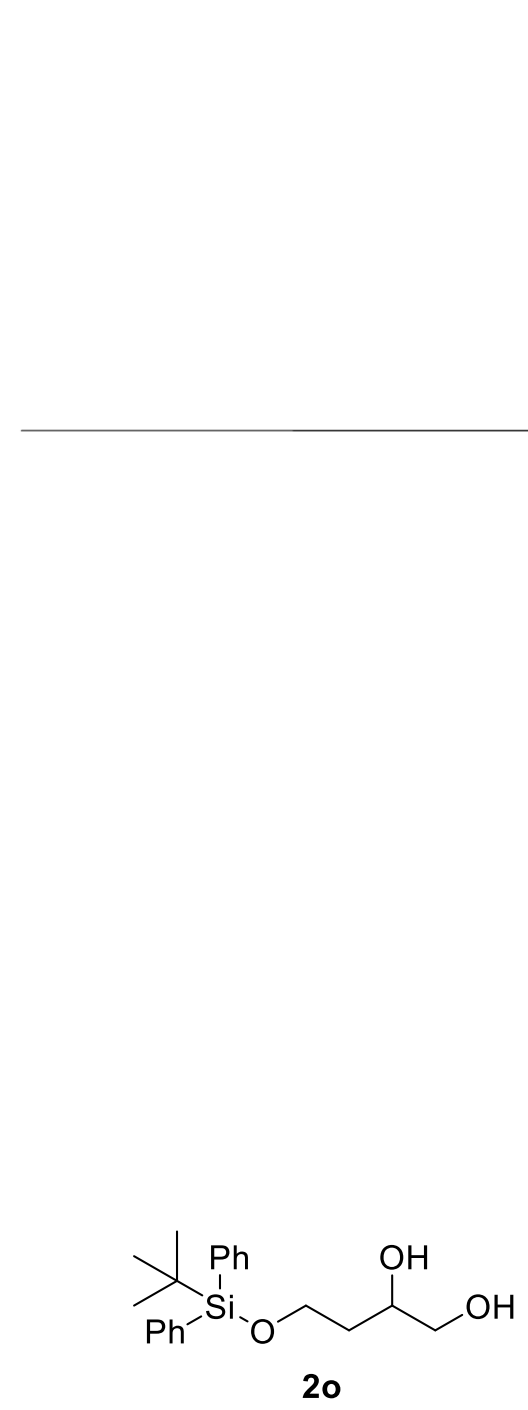

$\varepsilon 乙 8 ` Z 9$

$\angle 20 \angle 9$

ZL8.LL

$0 S 0^{\circ} \angle L$

$09 \varepsilon^{\circ} \angle L$

$\angle 89^{\circ} L$

6tเ:8ZL

เ乙乙0ع

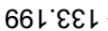

69乙'ยहL

$0 † 8 \cdot 9 \varepsilon$

9G8 $9 \varepsilon \downarrow$

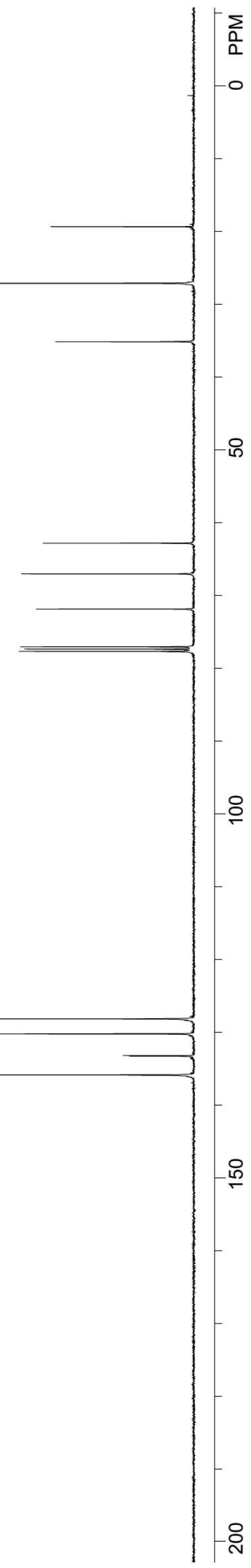



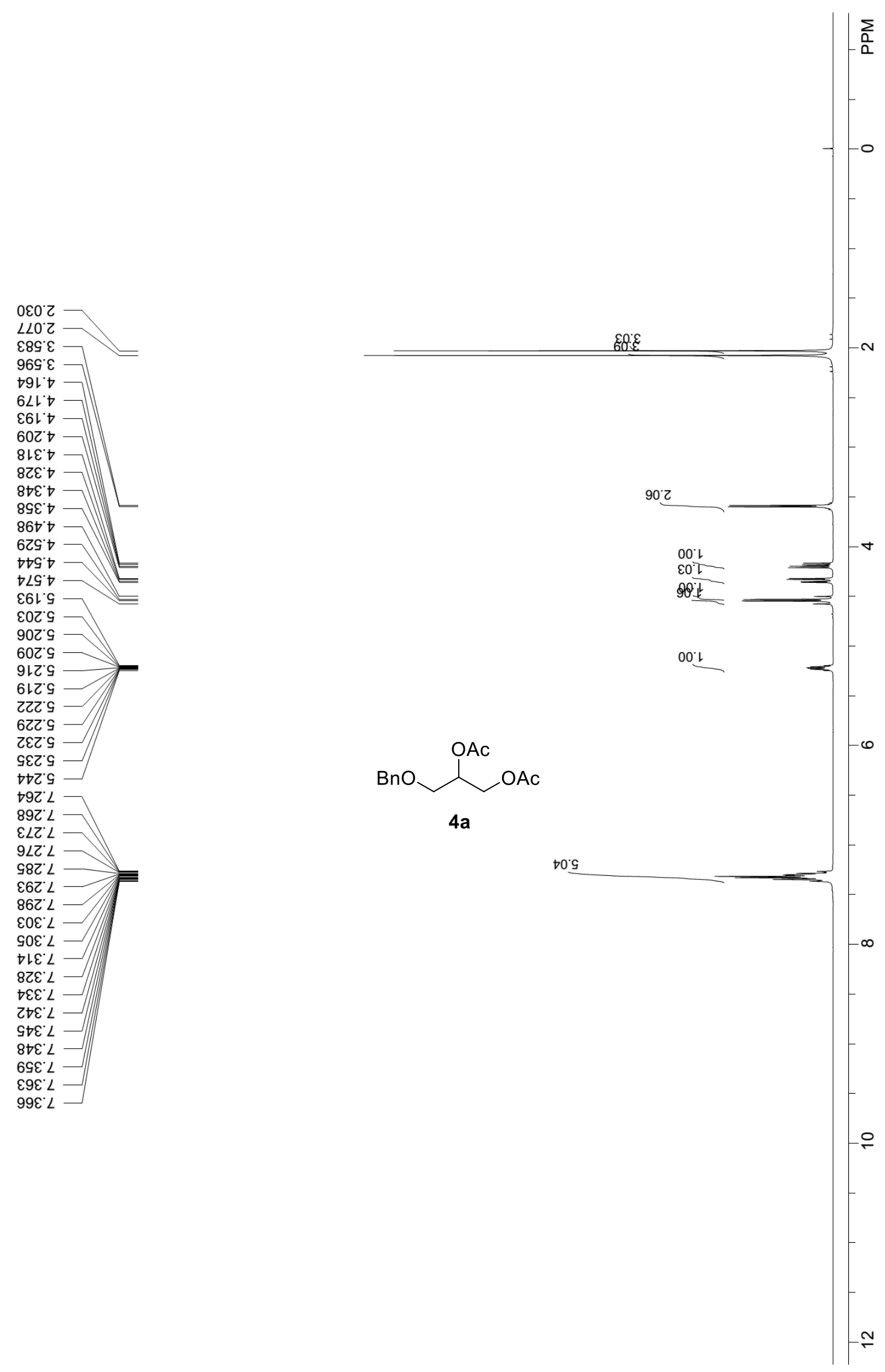

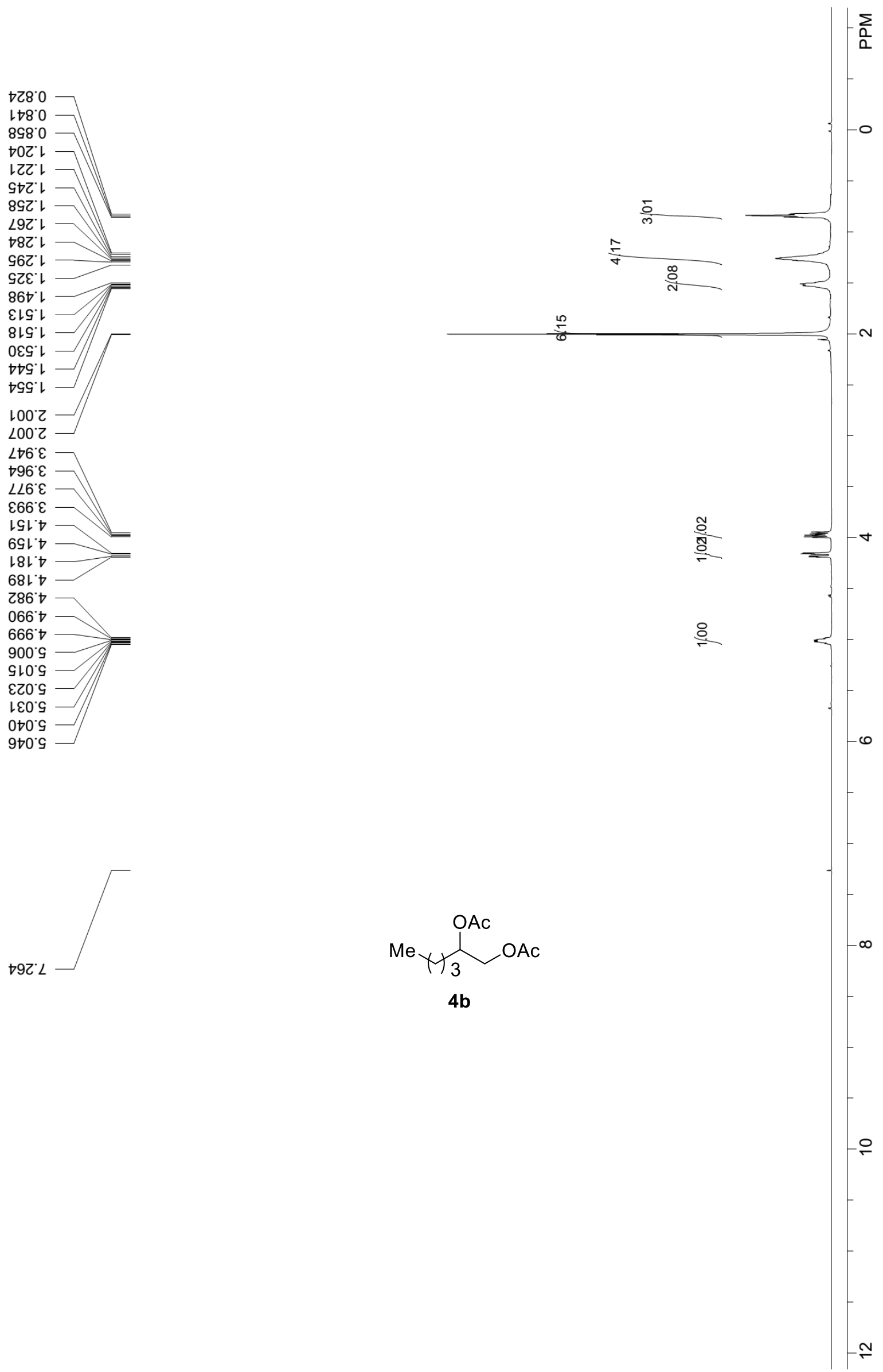

S-70 

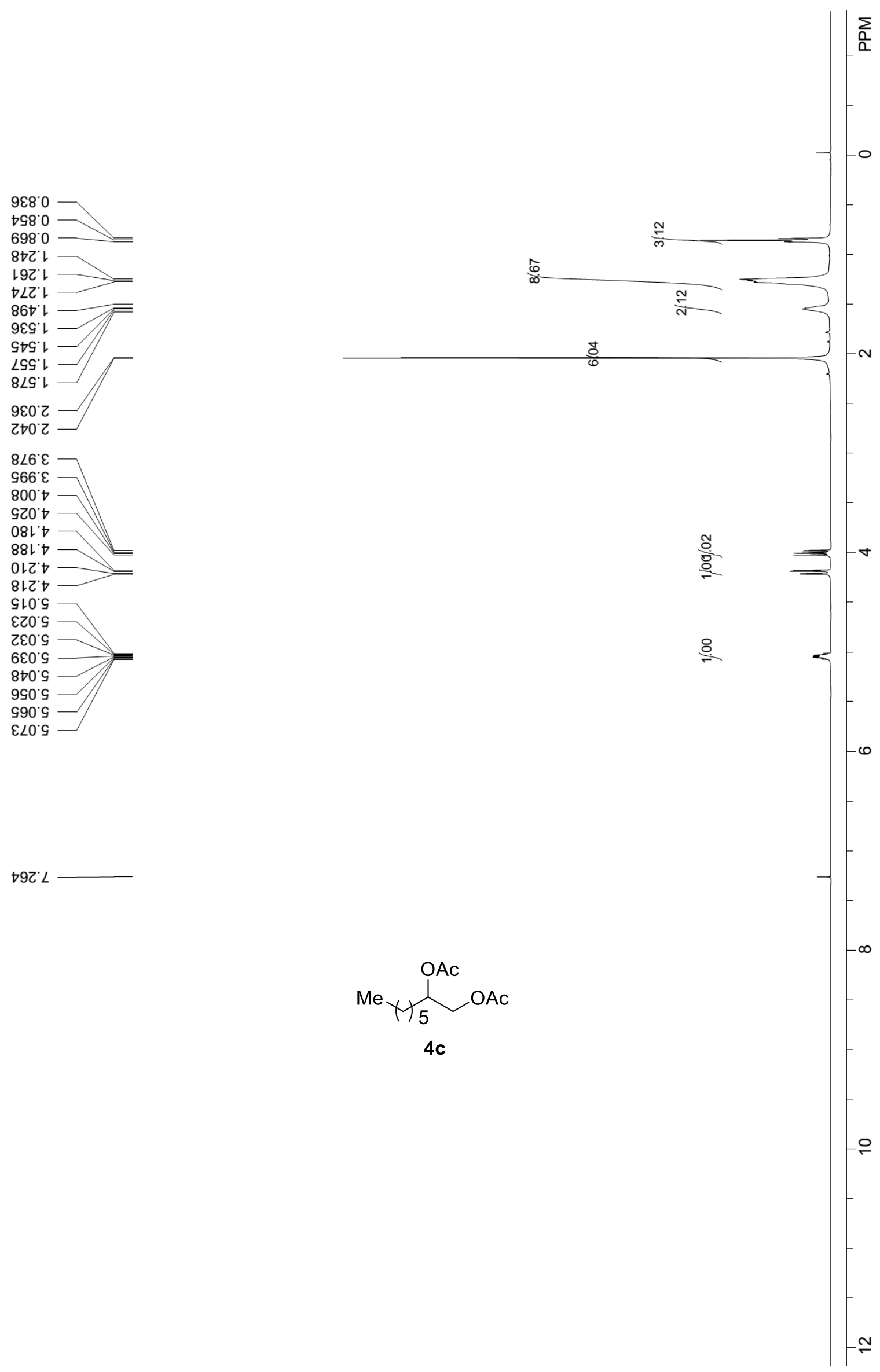

S-71 

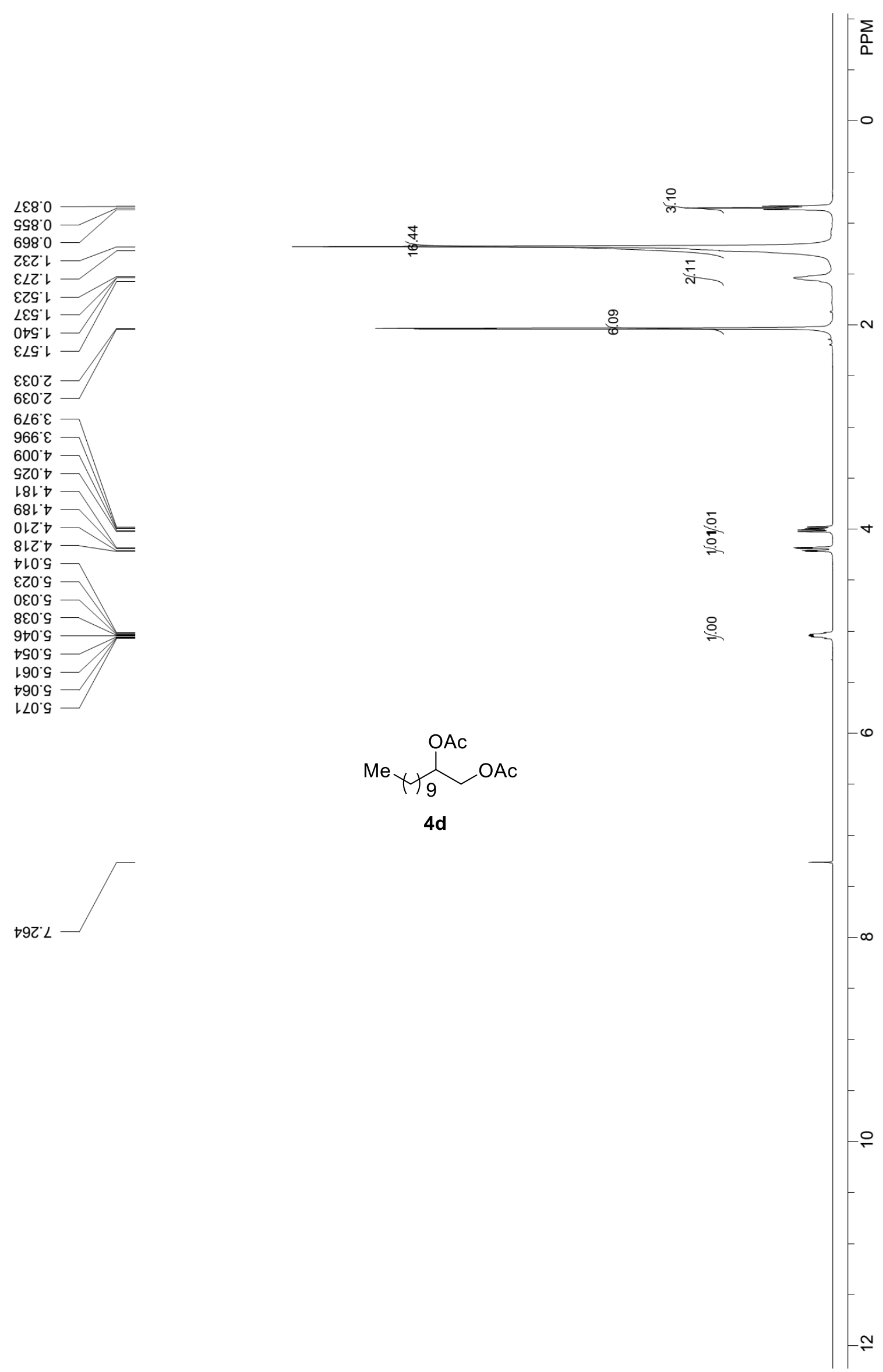

S-72 

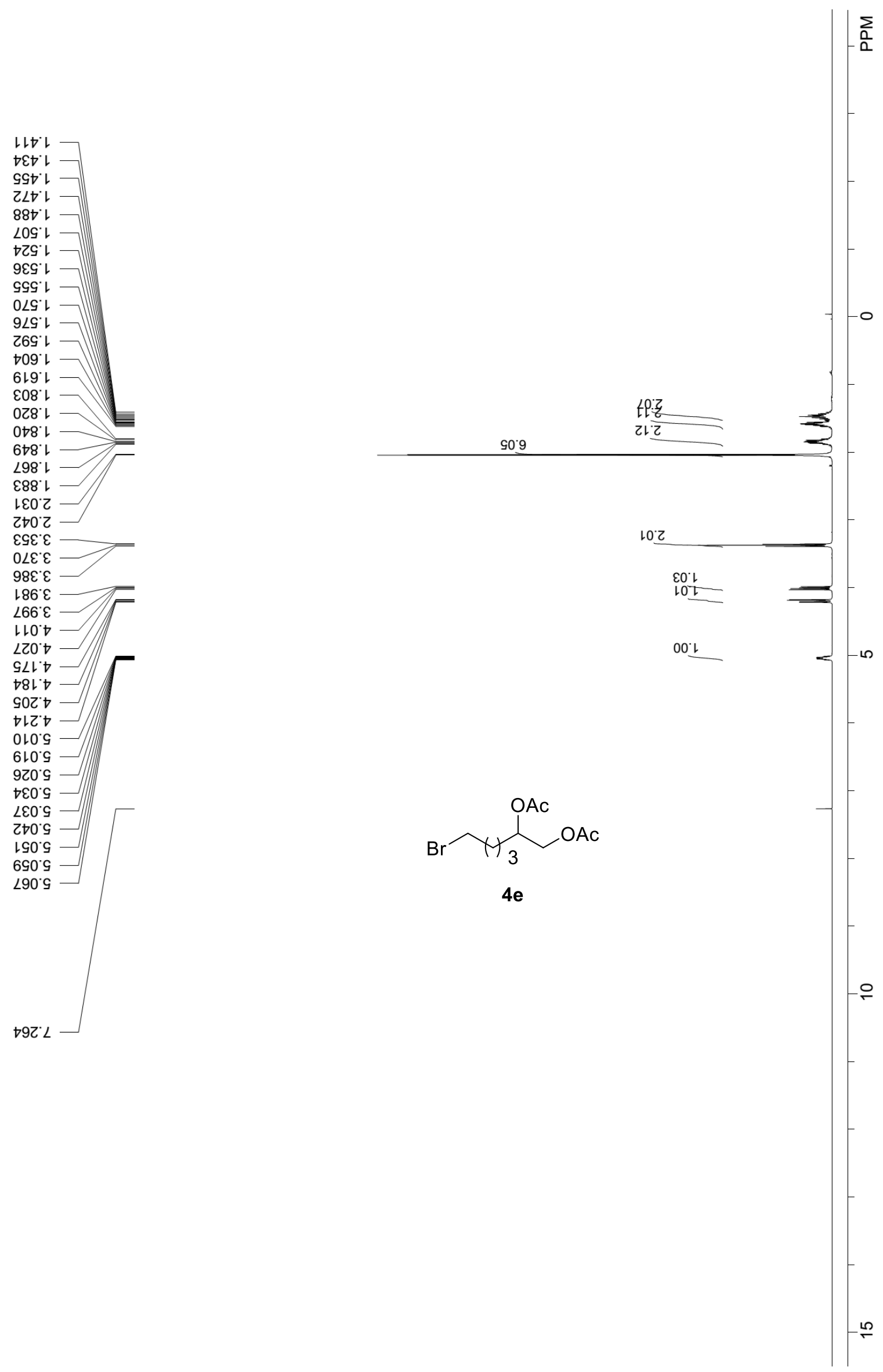

S-73 
$098^{\circ} 0 Z$

$6 \varepsilon レ$ レ

† ${ }^{\prime} \varepsilon 乙$

乙88 $6 乙$

† $\mathcal{E}^{\prime} 乙 \varepsilon$

$\neg \angle \mathcal{E}^{\prime} \varepsilon \mathcal{E}$

266'

LOZ'LL

SEO $L L$

$09 \varepsilon^{\circ} L L$

$6 \angle 9^{\circ} L L$

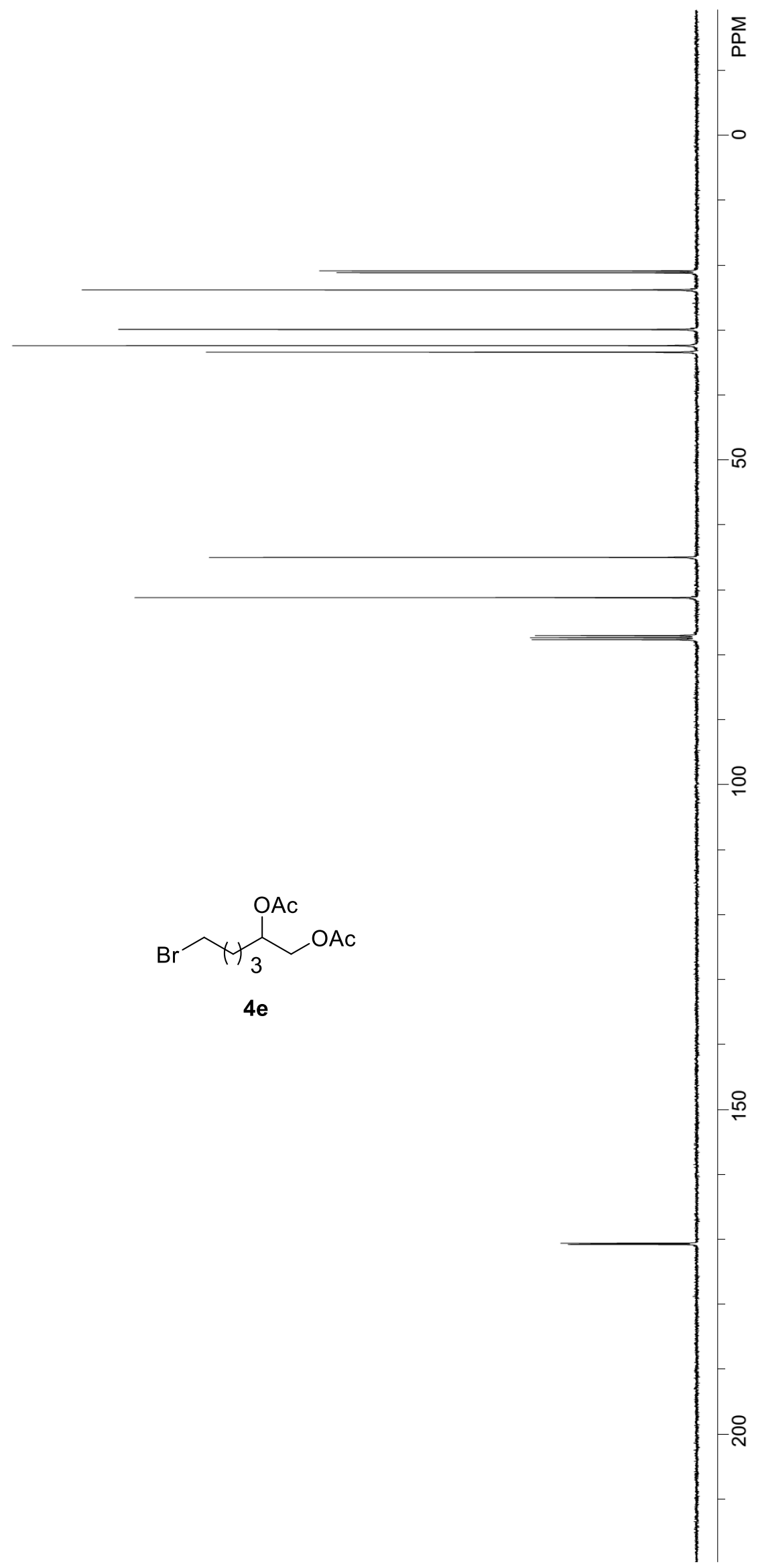




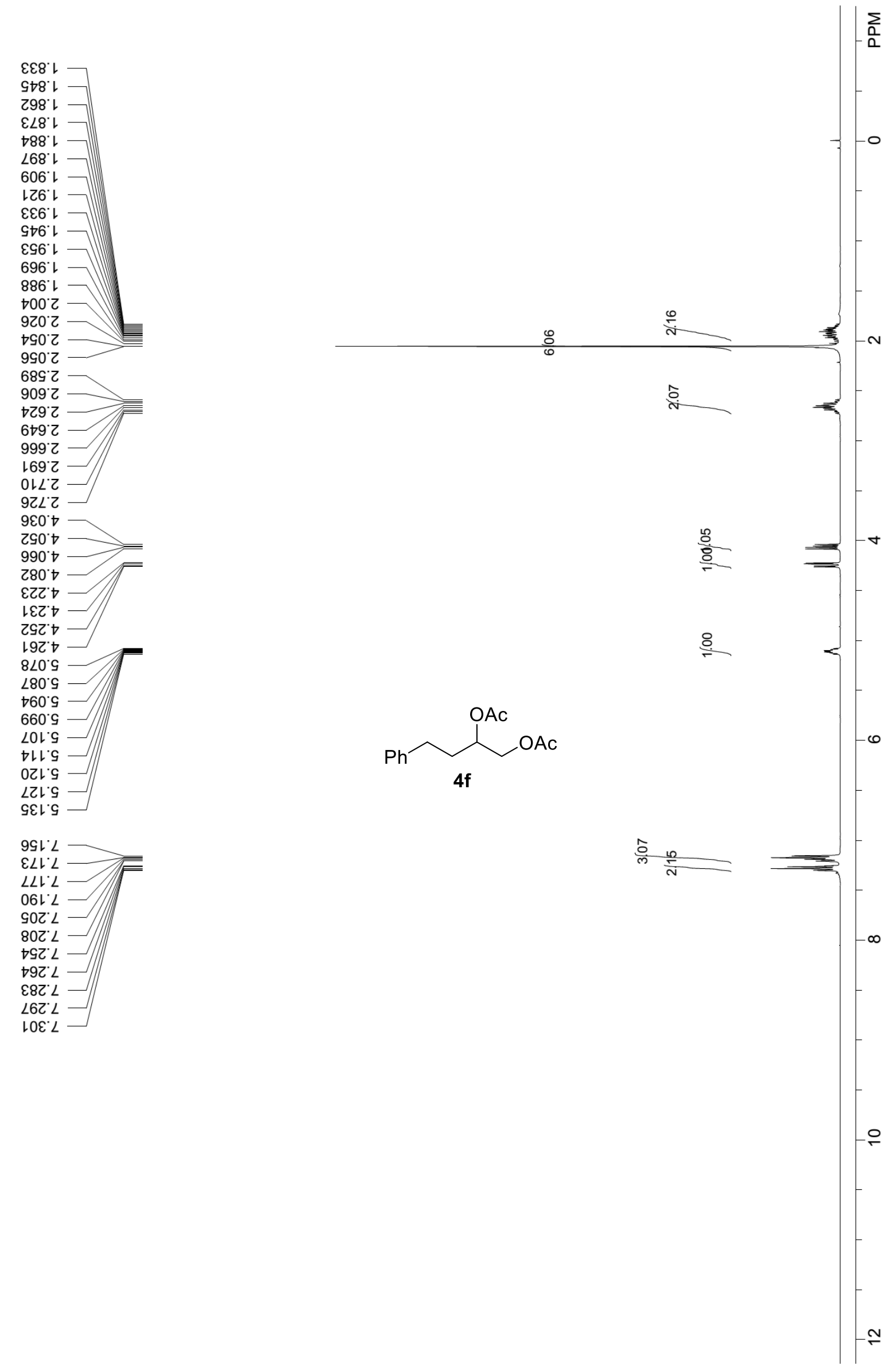


$000^{\circ}$

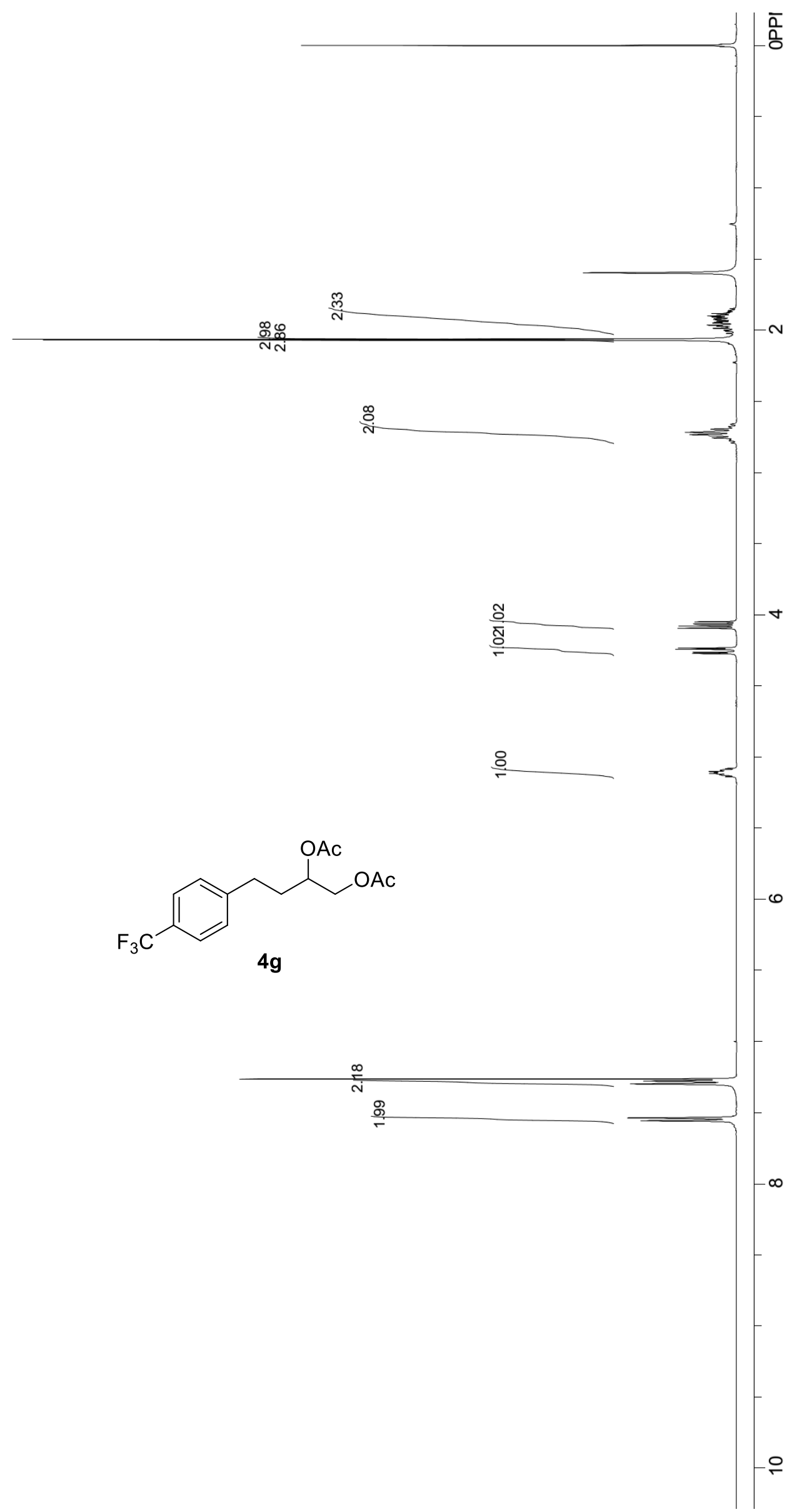

S-76 
$\downarrow \varepsilon 6^{\circ} 0 Z$

ย9เレ乙

$\angle 99^{\circ} L$

0ง\& 乙ะ

เ0เ.99

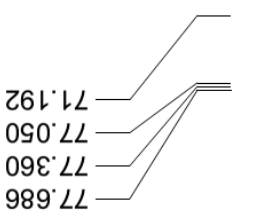

†乙乙 ६乙レ

\&S9' $9 Z 1$

889' GZL

EZL'GZL

6SL'GZL

$\angle L 6 G Z L$

$\angle \downarrow \varepsilon^{\circ} 8 Z L$

999.8Z

I96. 82 L

$\angle 86^{\circ} 8 \mathrm{ZL}$

8LE'6ZL

98ะ'งtr

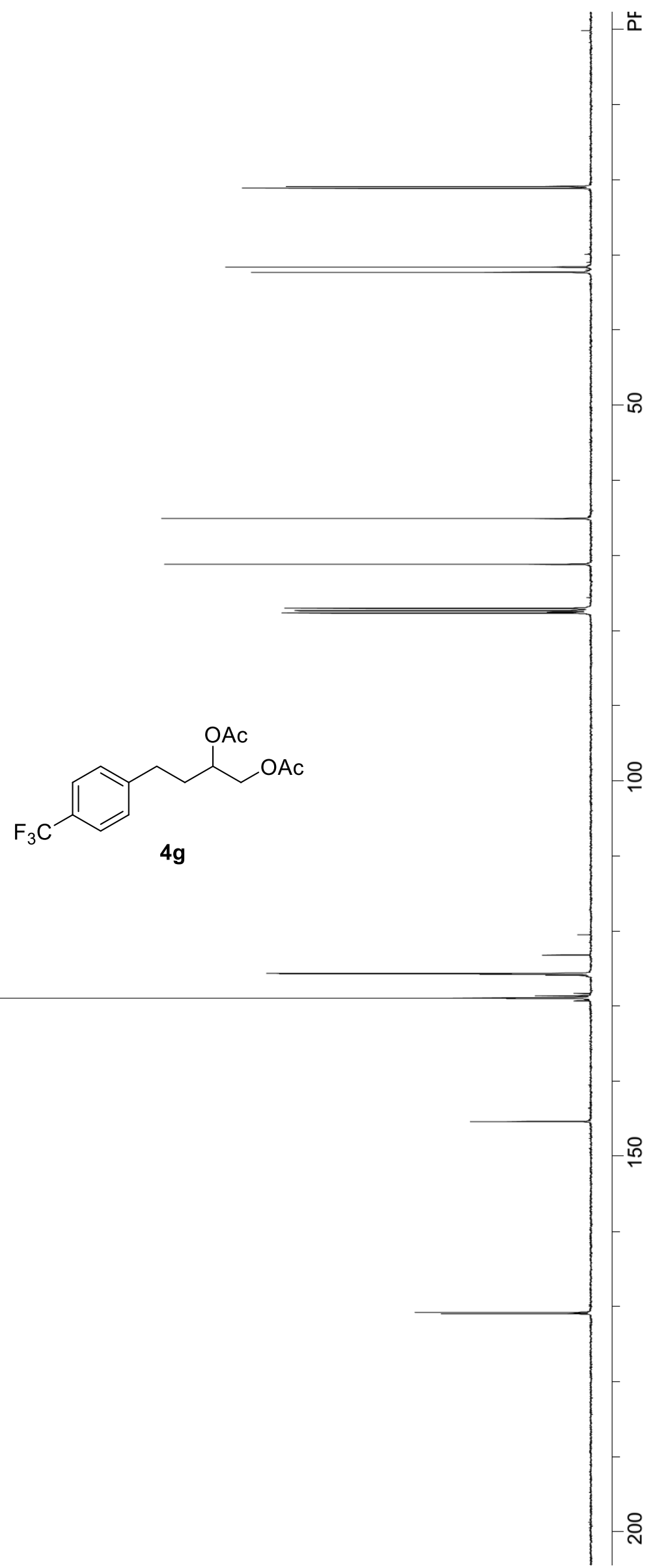

$4 g$

$\checkmark 6 L \circ 0 \angle L$

ZL6 $0 \angle L$

S-77 


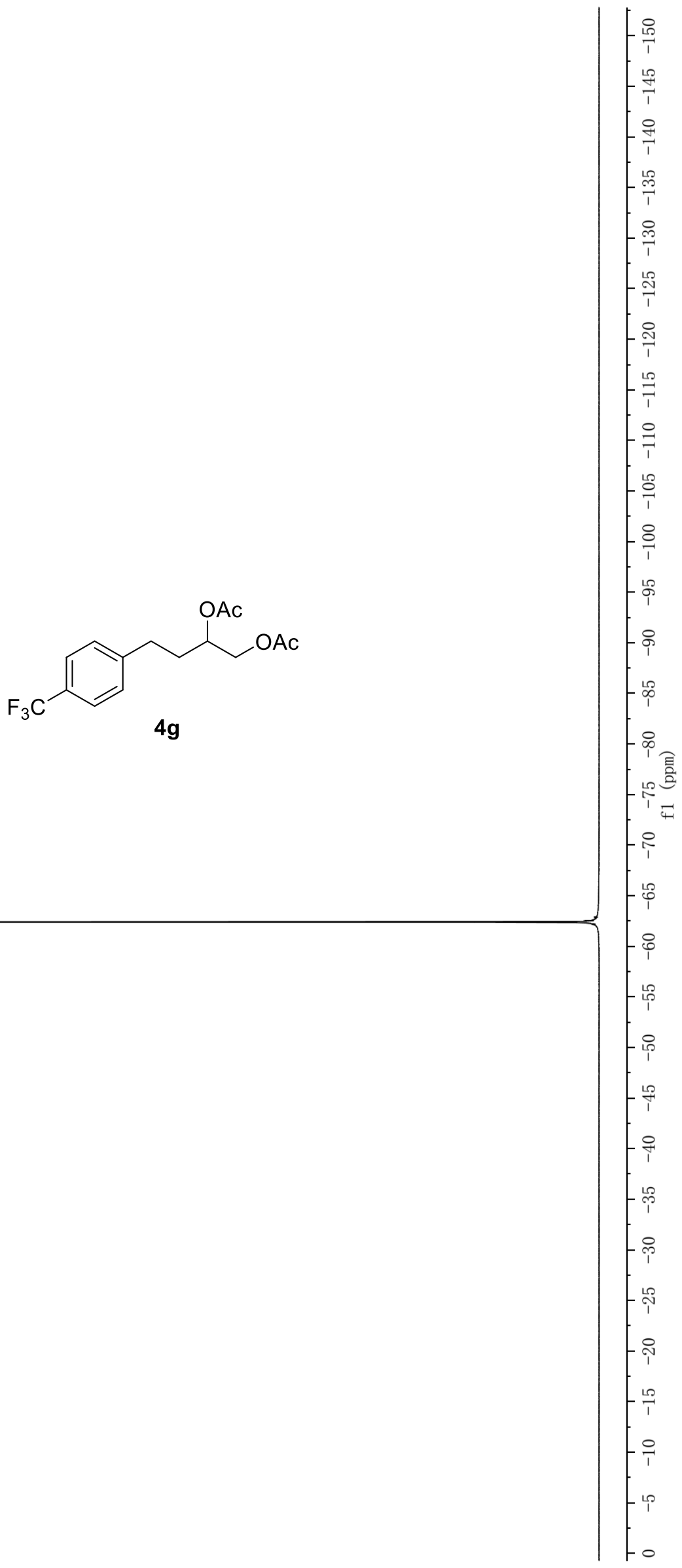

S-78 

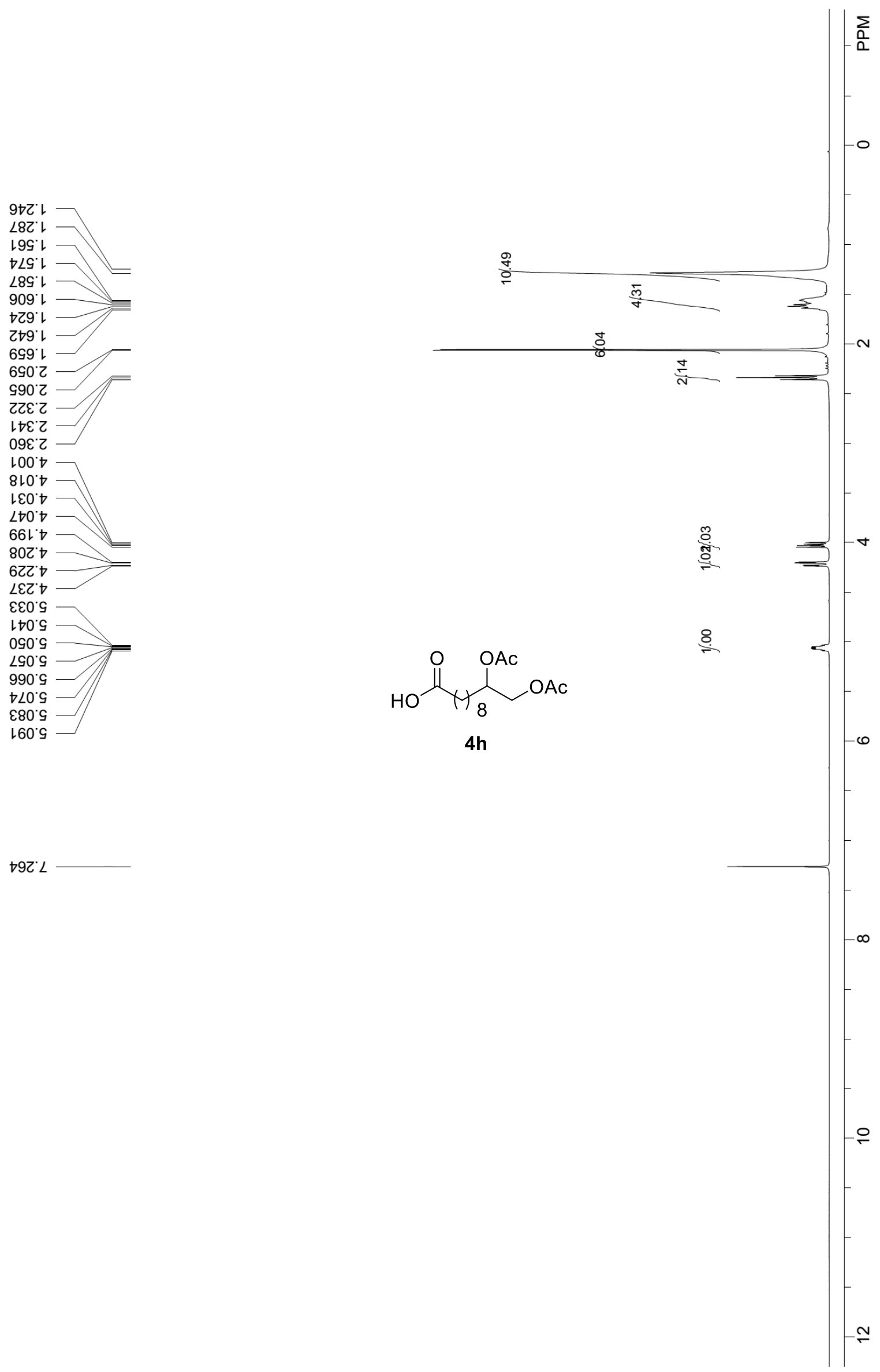

S-79 

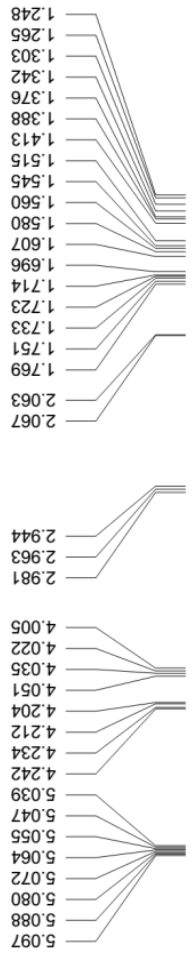

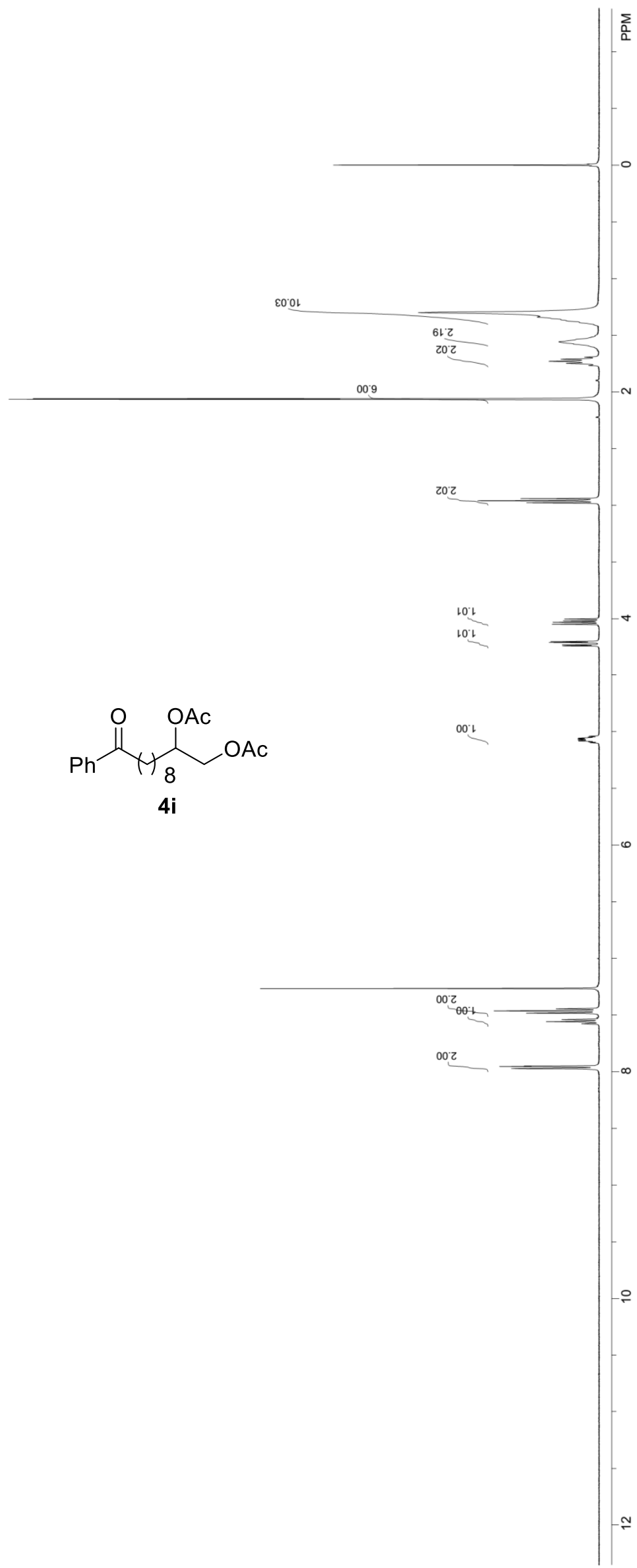

$t 9 Z^{\prime} L$
$\varepsilon t t^{\prime} L$
$Z 9 t^{\circ} L$

$29 t^{\circ} \mathrm{L}-$
$28 \mathrm{t}^{\circ} \mathrm{L}$ -

$280^{\circ} L$
$88 G^{\circ} L$
$L t G^{\circ} L$

$I t S^{\circ} L$
$\angle S S^{\circ} L$

$\angle S S^{\circ} L$
Z9G $L$

SLS'L

656.2
$\angle L 6 L$ 
$\angle 66^{\circ} 0 Z$

$96 Z^{\prime}\llcorner 乙$

$Z \nabla G^{\circ} \downarrow Z$

$\varepsilon เ \varepsilon^{\prime} G Z$

$6 \angle t 62$

$\nabla Z 9^{\circ} 6 Z$

$099^{\circ} 62$

$\varepsilon Z 6^{\circ} 0 \varepsilon$

†08 $8 \varepsilon$

0૬ع 99

$608 . L$

$\forall \varepsilon 0^{\circ} \angle L$

$09 \varepsilon^{\circ} L L$

$\angle \angle 9^{\circ} \angle L$

$\varepsilon 6 乙 8 Z 1$

$\varepsilon 6 L^{\circ} 8 \mathrm{LL}$

60 เ $\varepsilon \varepsilon \downarrow$

ZLE'LEL

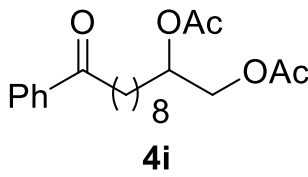

$978.0 \angle 1$

$866^{\circ} 0 \angle L$

$8 L L^{\circ} 00 Z$ 

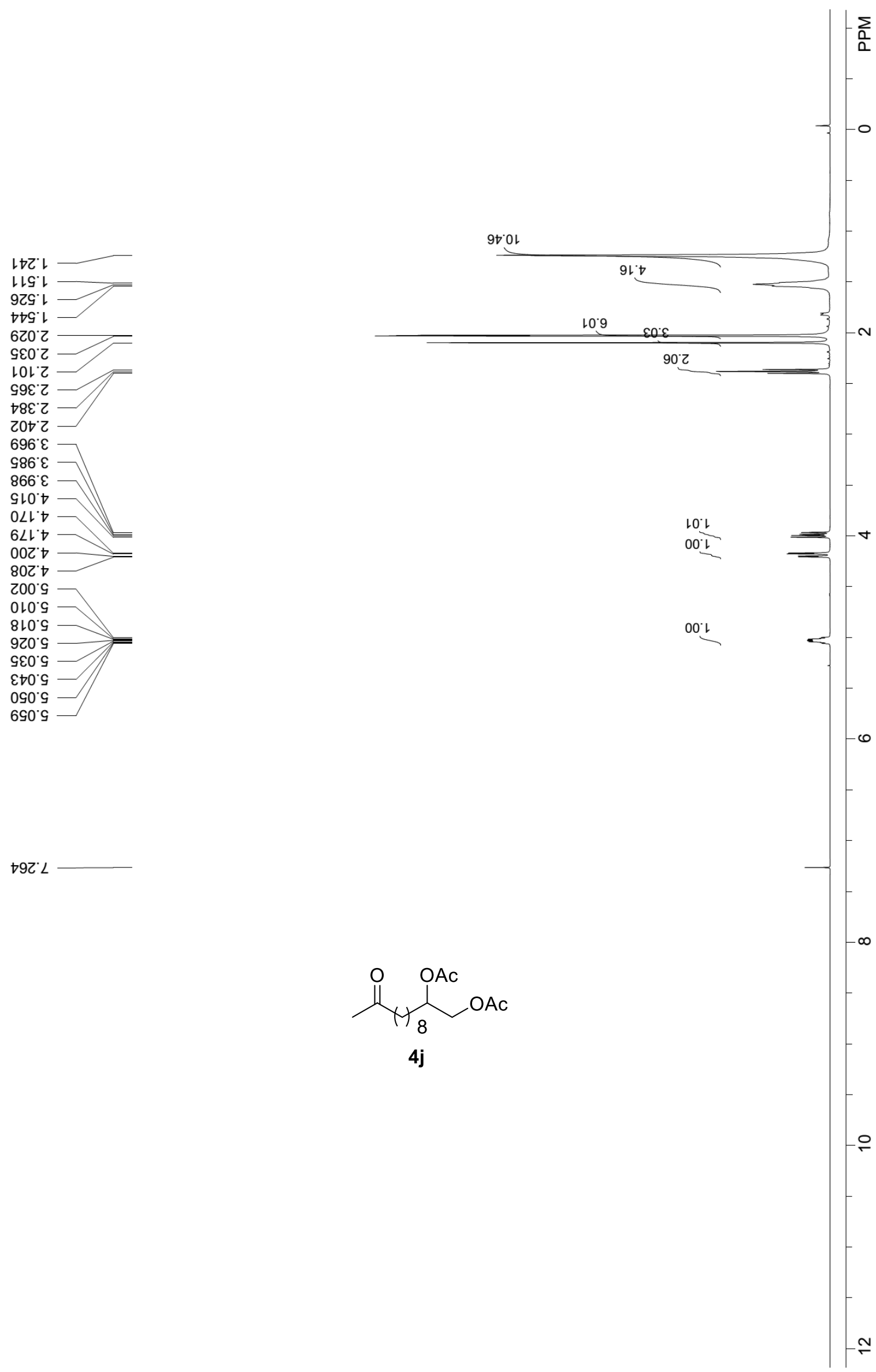

S-82 
๖๐レて

$6 \varepsilon \varepsilon^{\circ}$

$\varepsilon \nabla 0^{\circ} \nabla 乙$

$\downarrow \varepsilon \varepsilon \cdot \varsigma 乙$

S†ย' 62

$\downarrow 9 \nabla^{\circ} 6 z$

109.62

हEG 62

จ००० $0 \varepsilon$

$8 Z 6^{\circ} 0 \varepsilon$

6เO๋t

๑ะ' 99

हZ8. $L$

9E0 $L$

$09 \varepsilon^{\circ} \angle L$

$9 \angle 9^{\circ} \angle L$

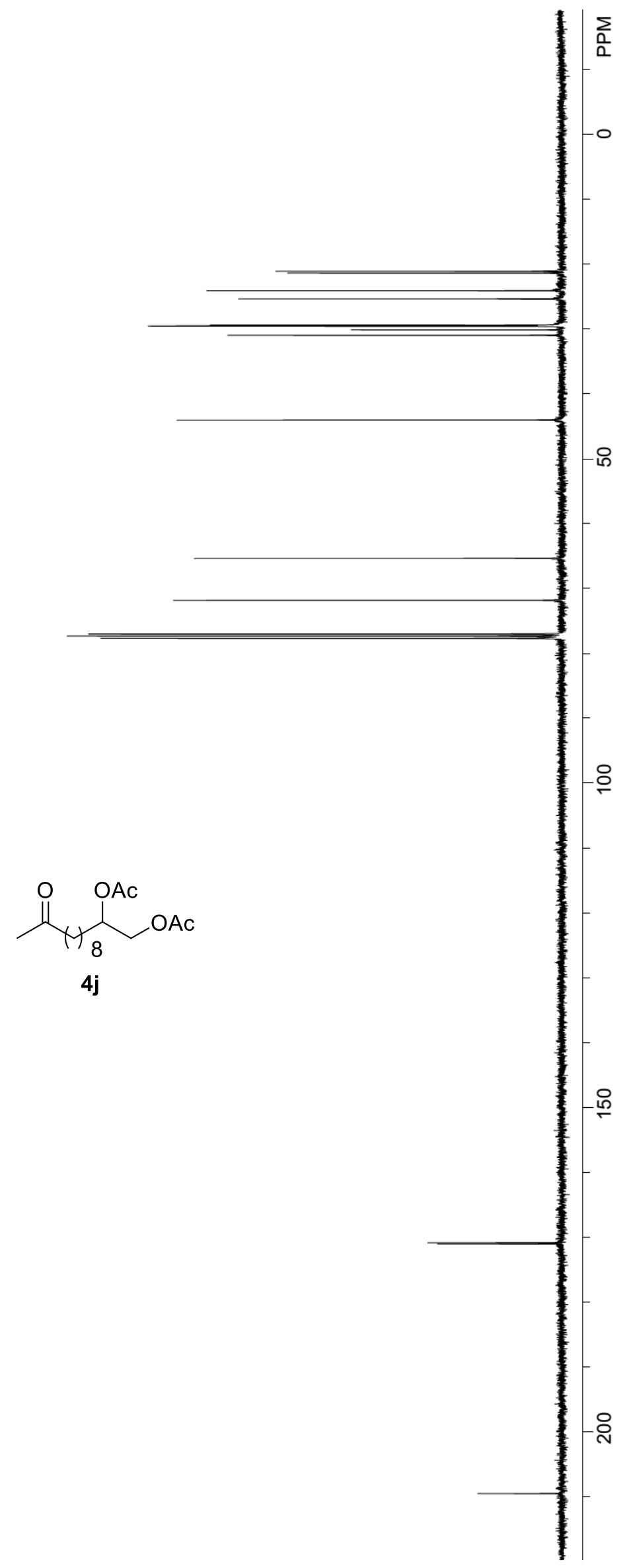



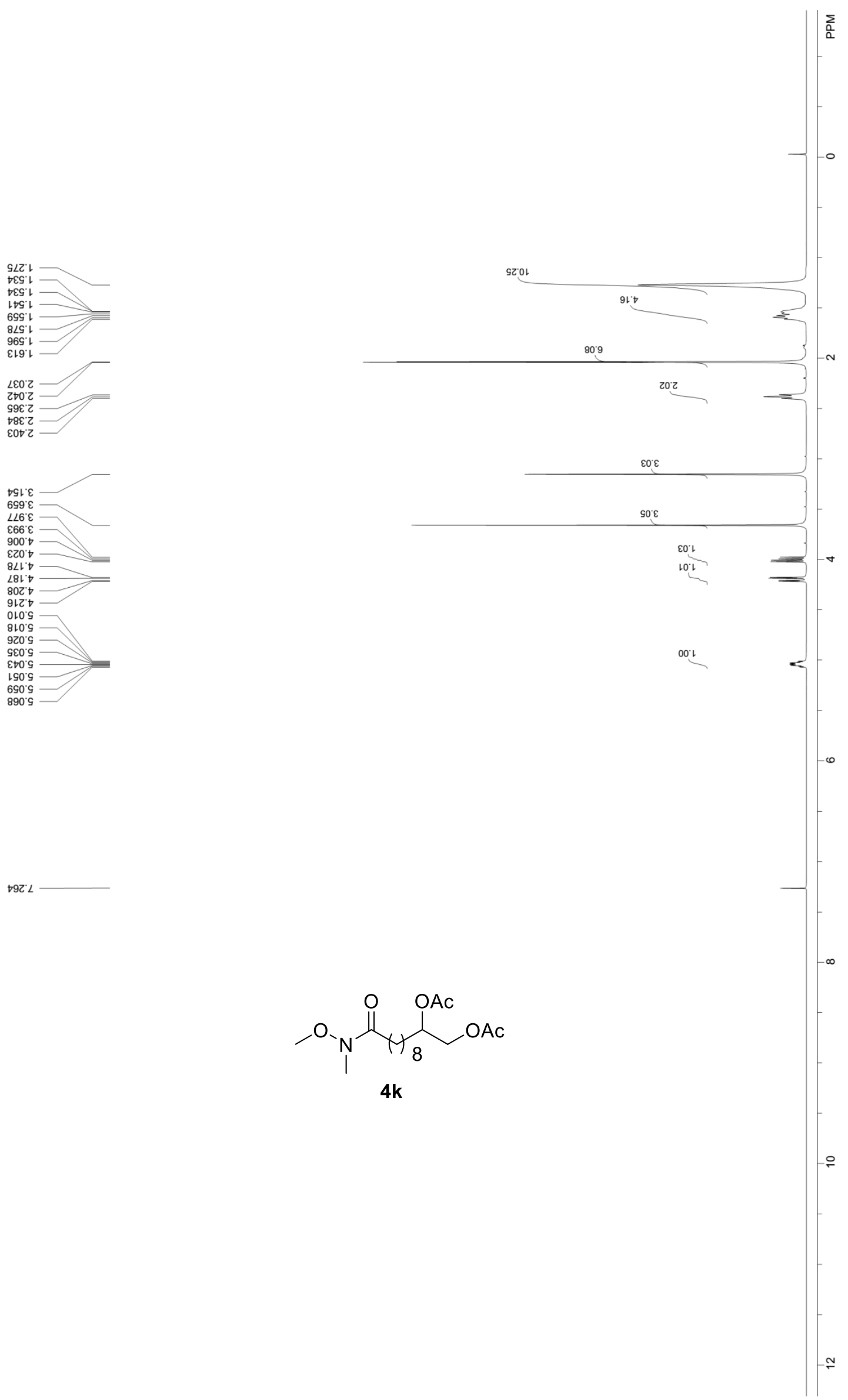

S-84 


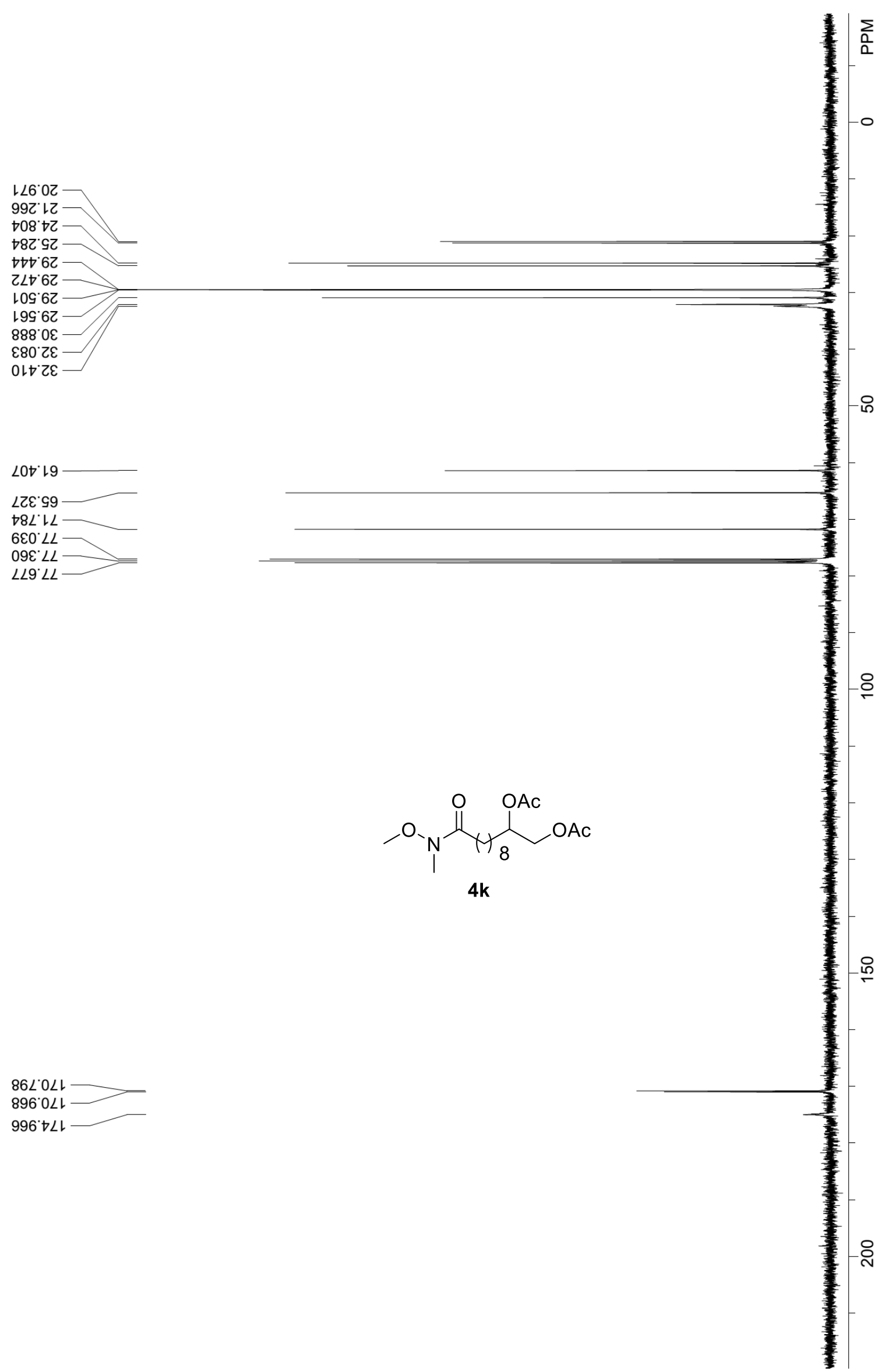



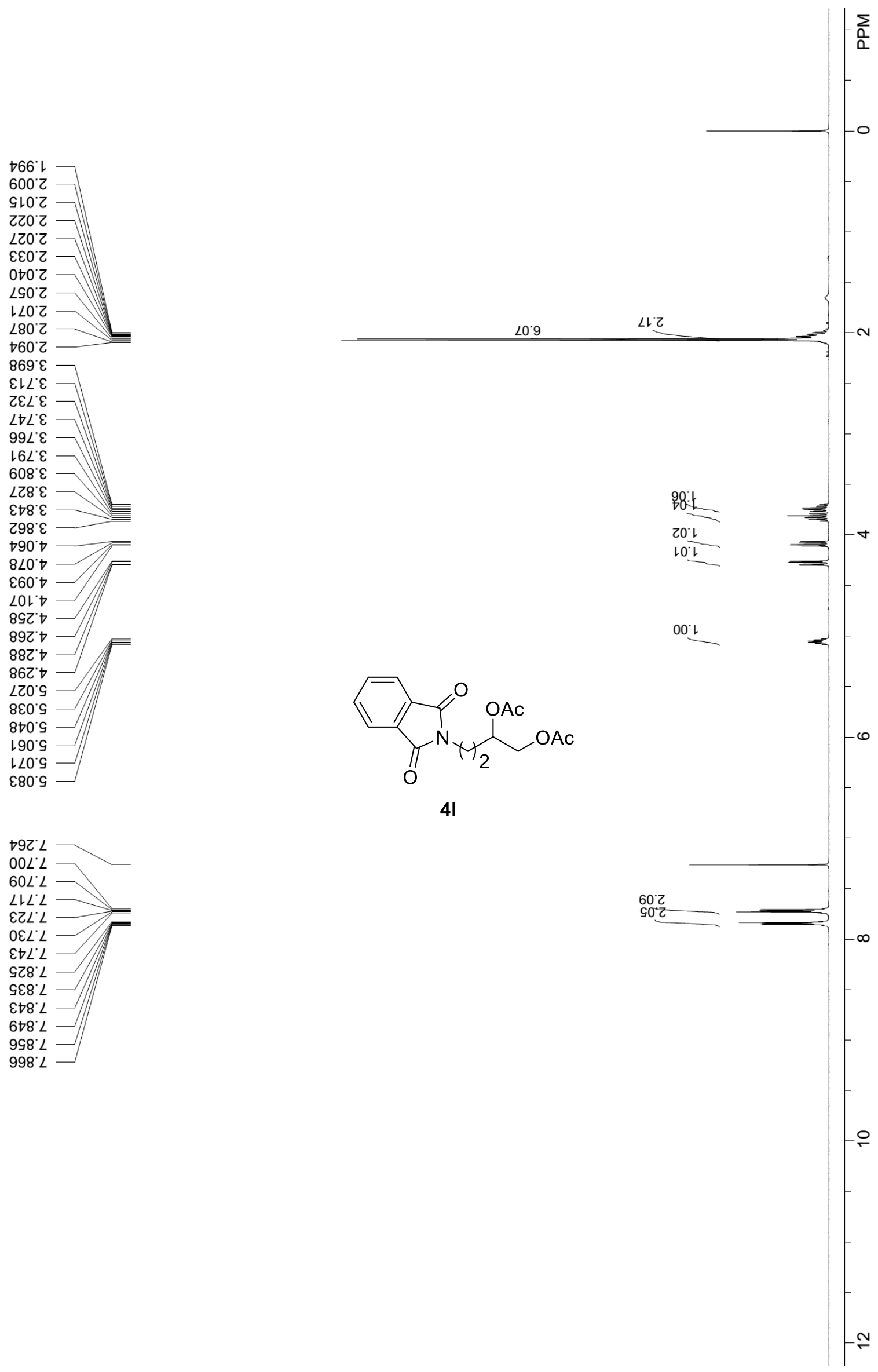
$996^{\circ} 02$

$181 \cdot 12$

$\varepsilon 09^{\circ} 62$

$\angle 8 \varepsilon^{\circ} \triangleright \varepsilon$

乙†8 $ฑ 9$

$8 Z 269$

$6+0^{\circ} \mathrm{LL}$

$09 \varepsilon^{\circ} \angle L$

$989^{\circ} \mathrm{L}$

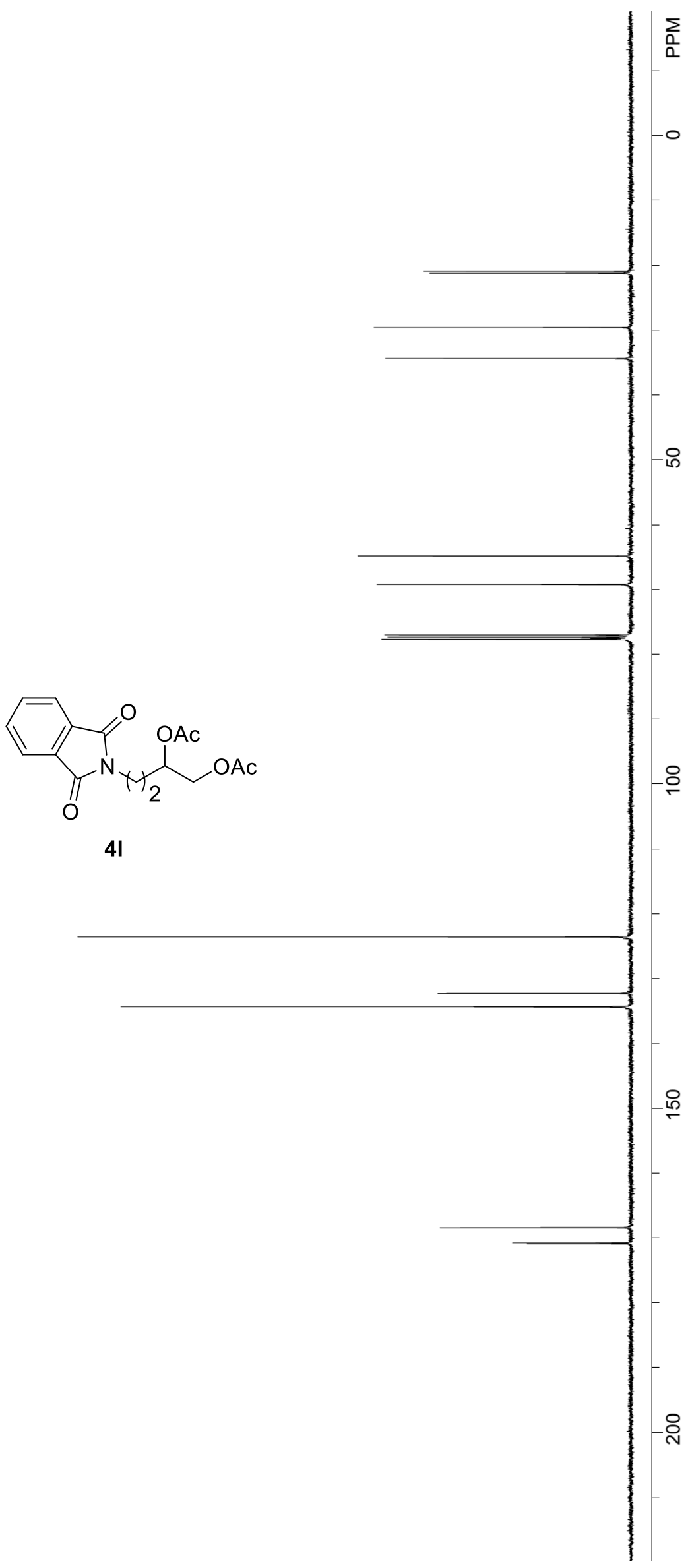

$9 l+89 k$

SLLOLL

$98^{\circ} 0<1$

$\varepsilon 97 ' Z \varepsilon$

$9\left\llcorner\varepsilon^{\prime} \triangleright \varepsilon\llcorner\right.$

8૬૬`६२Ь 

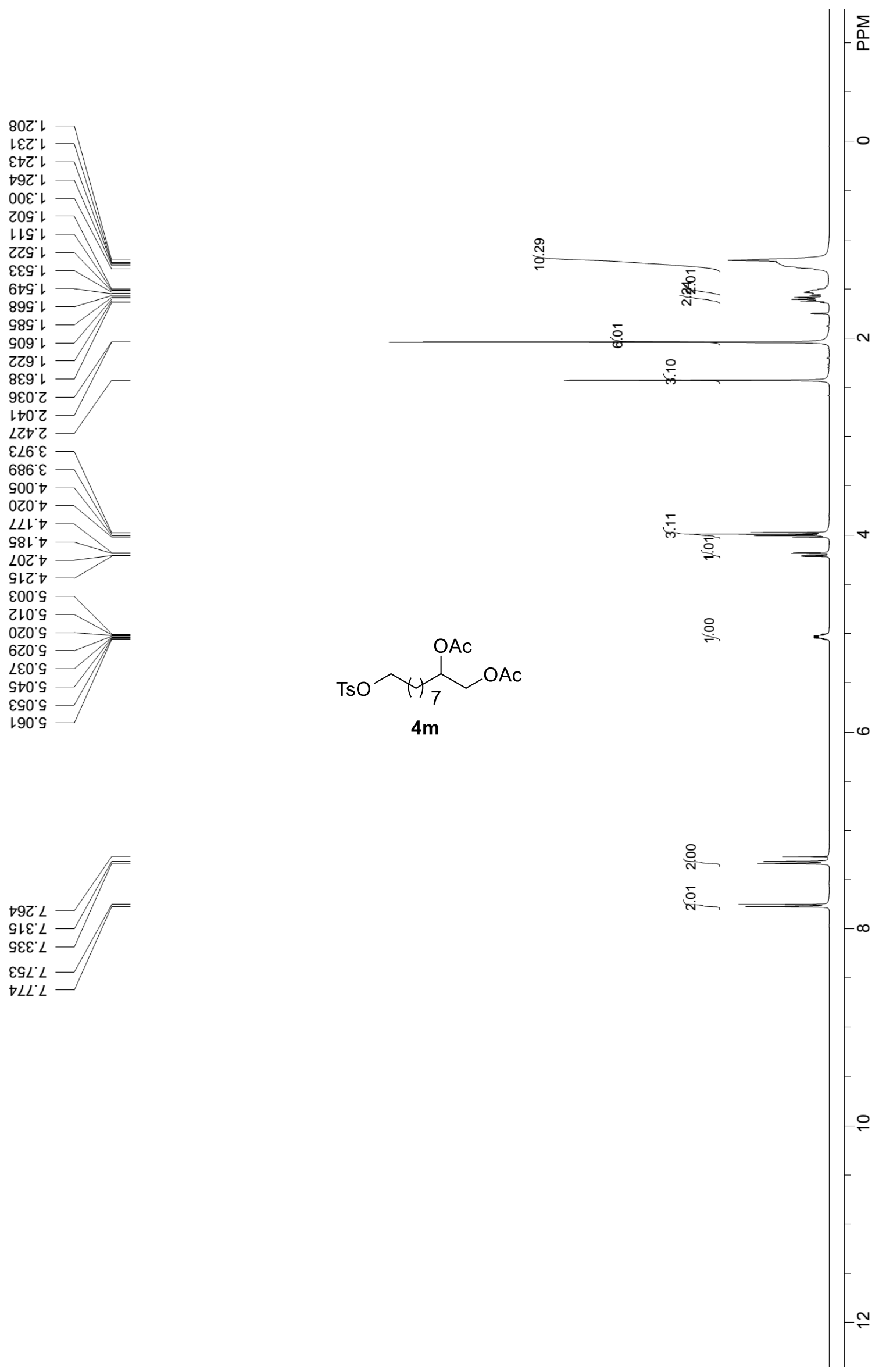

S-88 


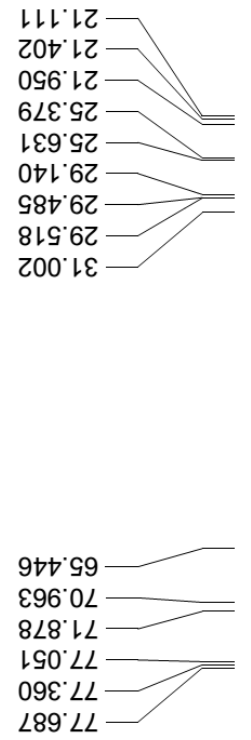

ह乙乙 8乙レ

0†レ'0ع

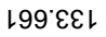

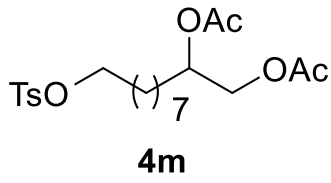

896レレ

$096^{\circ} 0 \angle \mathrm{L}$

เทレレL 

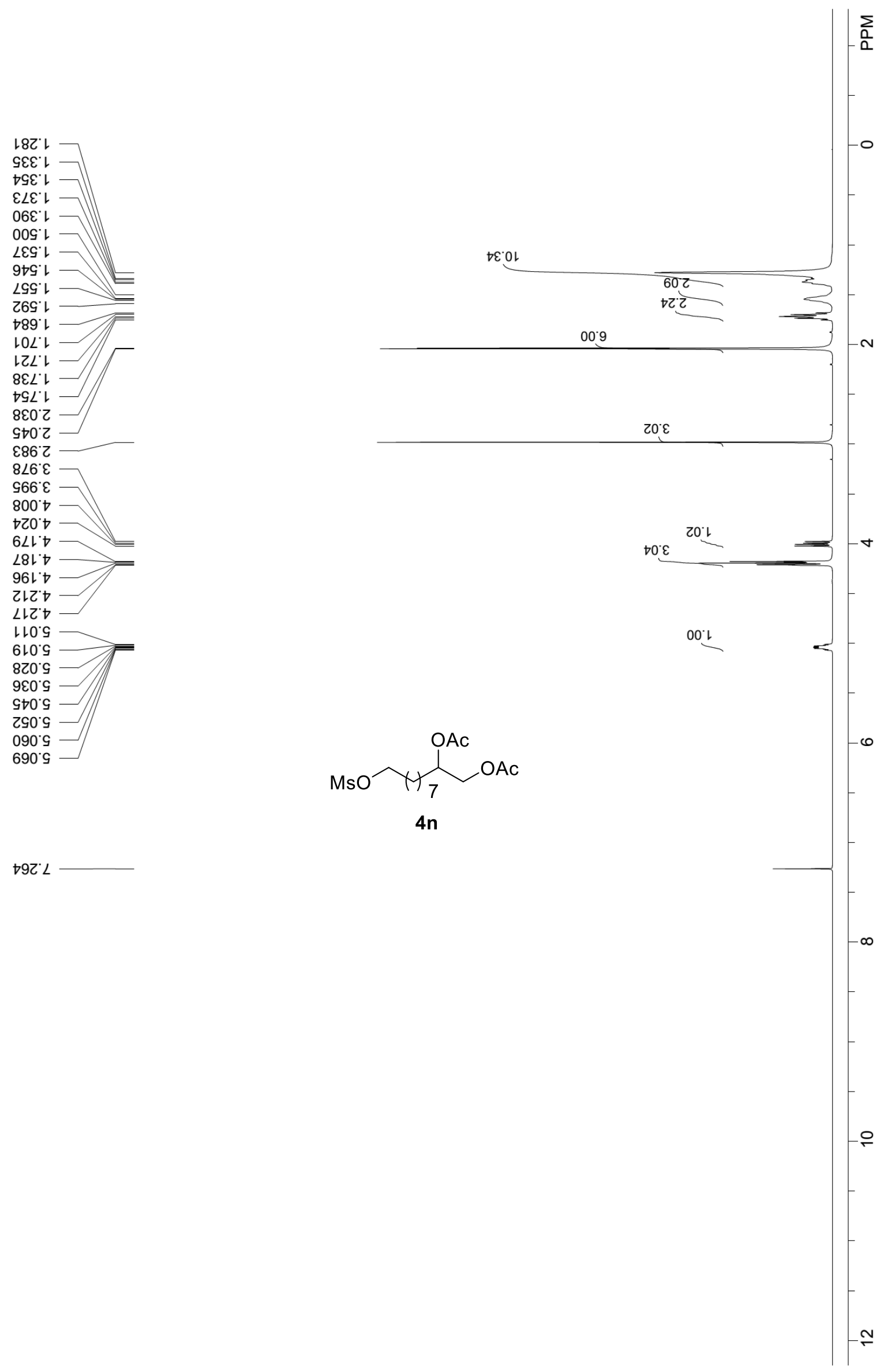


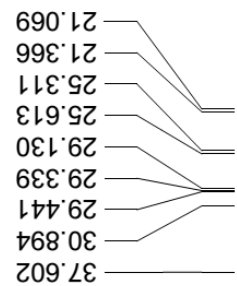

89\&'99

8Z७ 02

G9L'LL

I $00^{\circ} L L$

09E $\angle L$

$Z \angle 9^{\circ} L L$

$2 Z 6^{\circ} 0 \angle L$

$660^{\circ} L<1$

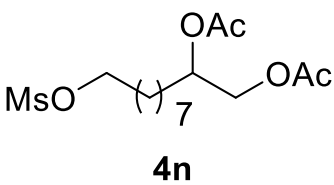



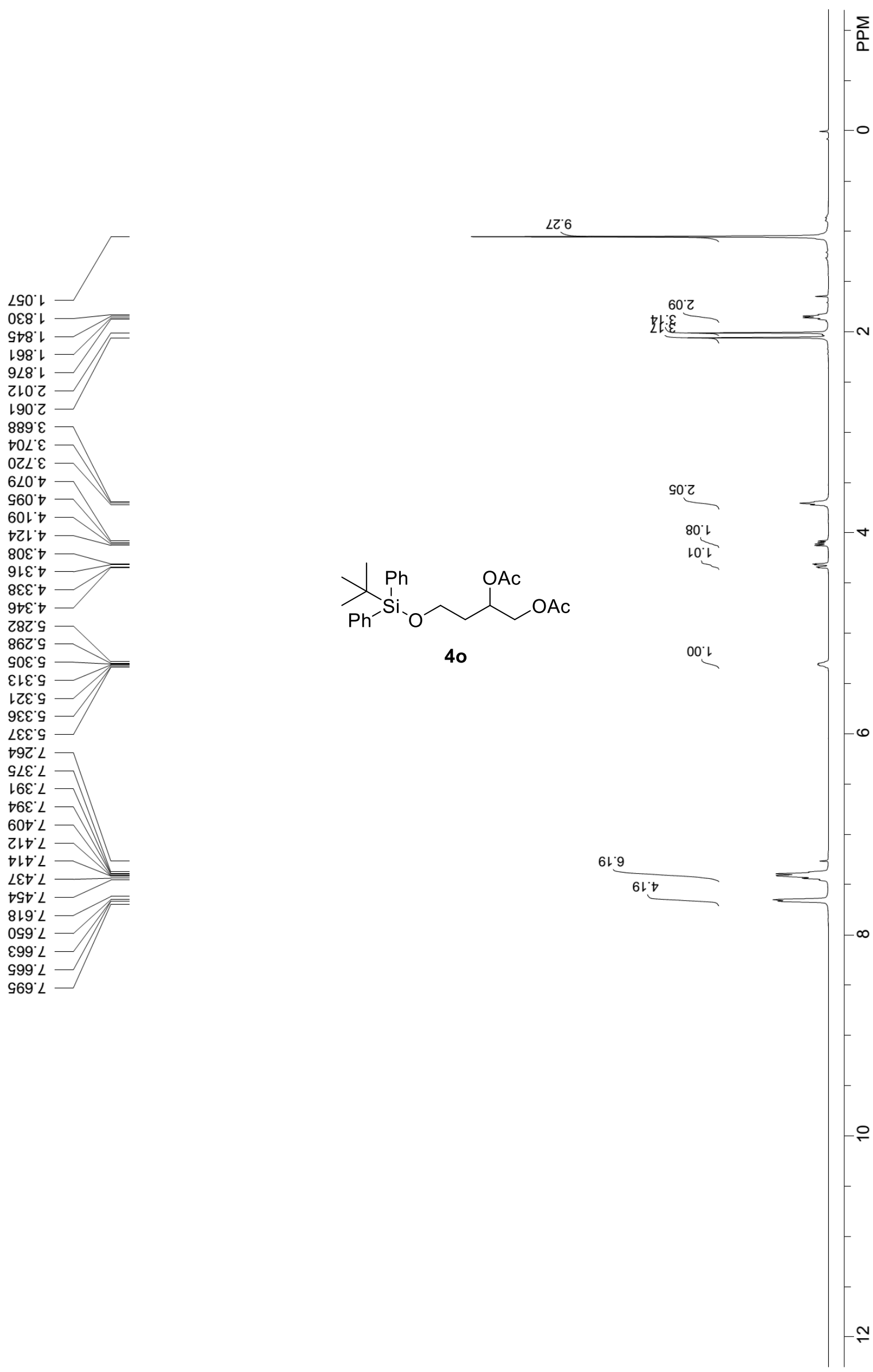
9Zt56

$960 . L$

$\varepsilon 乙 \varepsilon^{\circ}\llcorner 乙$

OLLLZ

$\angle 98^{\circ} \varepsilon \varepsilon$

$266^{\circ} 69$

乙૬G' 99

เ9t 69

$\varepsilon \varepsilon 0^{\circ} L L$

$09 \varepsilon^{\prime} L L$

SL9 $\angle L$

LE0 $8 Z L$

$\angle L O O E L$

S†L'E\&L

8L8 $\subseteq \varepsilon L$

$\angle 99^{\circ} 0 \angle L$

ISO $L L L$

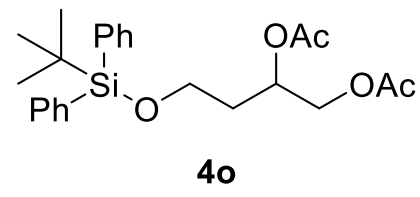



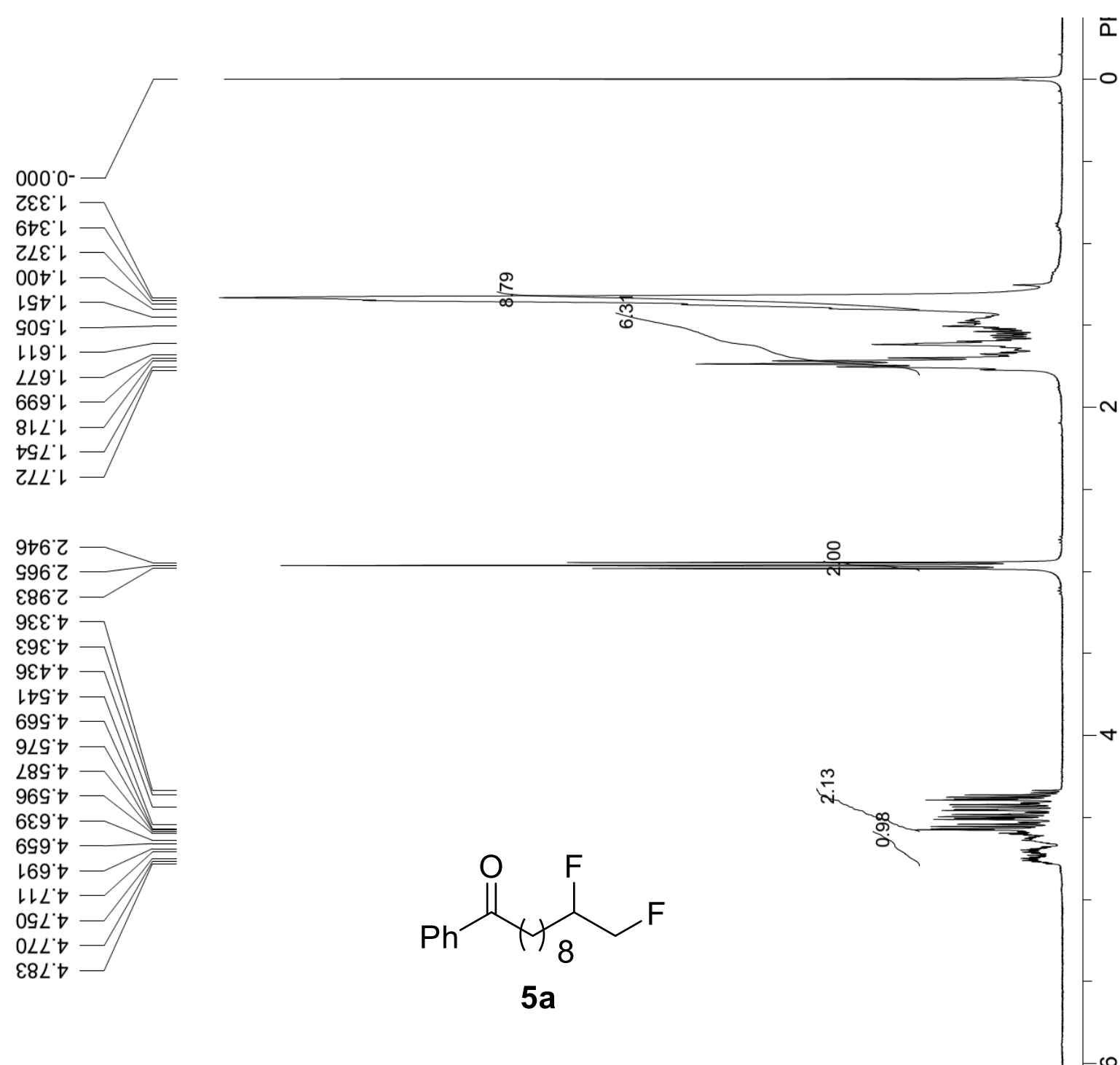

E9Z L

Z๐t L

$09 t^{\circ} \mathrm{L}$

$087^{\circ} L$

8ES $L$

999 $L$

$\nabla \angle S^{\circ} L$

$\angle D 6^{\circ} \angle$

IS6 $\mathrm{L}$

$696^{\circ} \mathrm{L}$

LL6. L

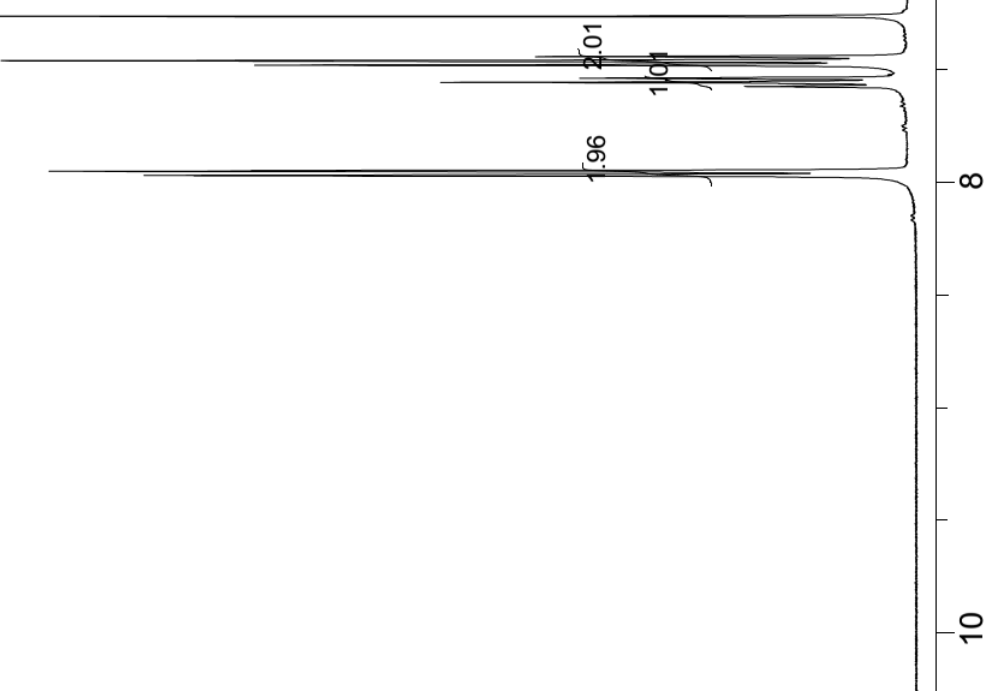




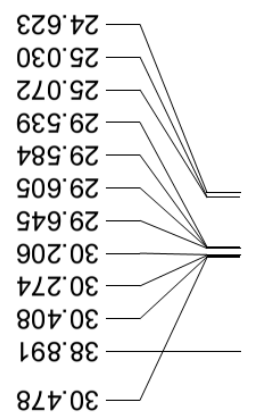

$8 \nabla 0 \angle L$

$09 \varepsilon^{\circ} \angle L$

$989^{\circ} \angle L$

$809^{\prime} \varepsilon 8$

เレL'\&8

$\downarrow \varepsilon 乙 ` \varsigma 8$

Z9t`98

レレ゙レ6

$20 t \cdot 16$

५Z6. Z6

เZI' $\varepsilon 6$<smiles>CC(CF)NC(=O)c1ccccc1</smiles>

$5 a$

$\neg 8 \varepsilon^{\prime} 8 Z \mathrm{~L}$

868. $8 Z$ L

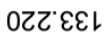

OSt $\angle E L$

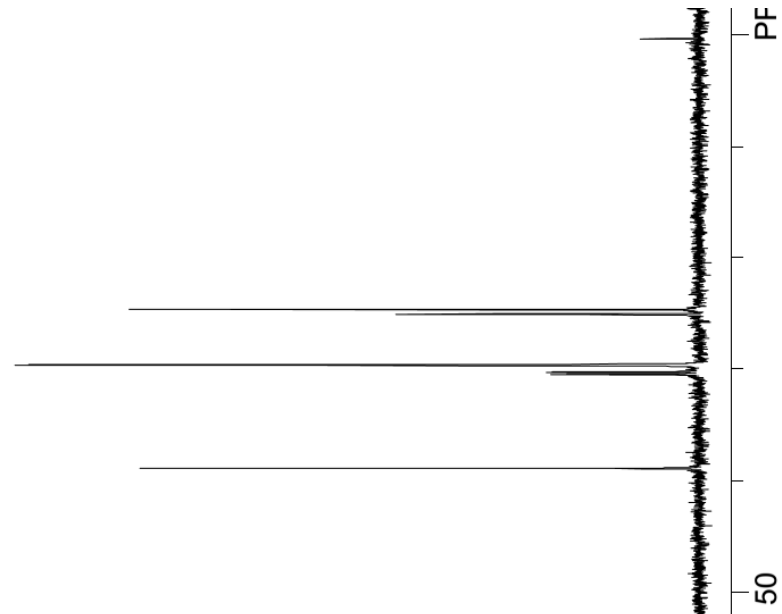


ย乙8 $6 乙{ }^{-}$

$\angle 8 L^{\prime} 6 Z Z^{-}-$

6ะ8'88เ-

$\varepsilon 08.88 \mathrm{~L}$

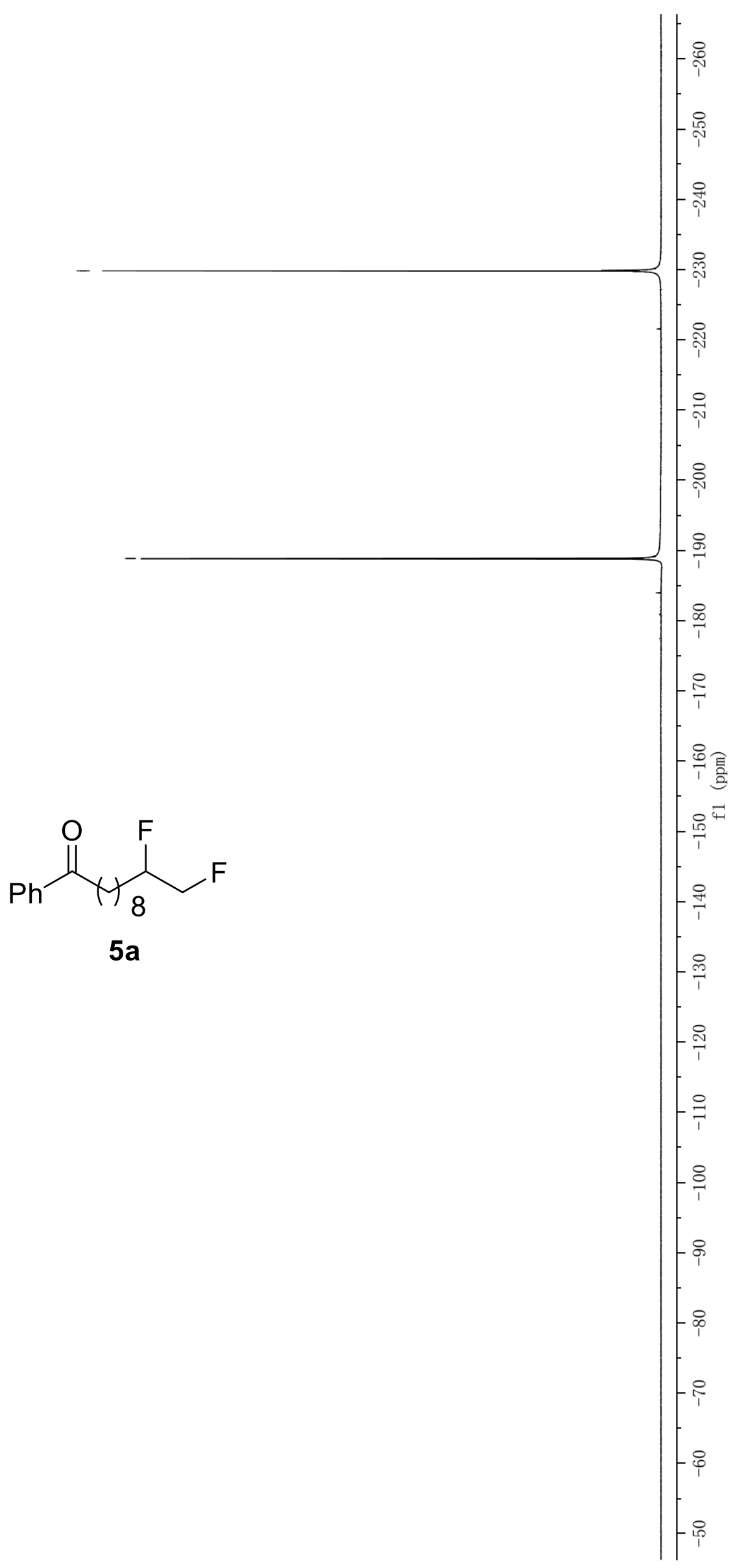

S-96 

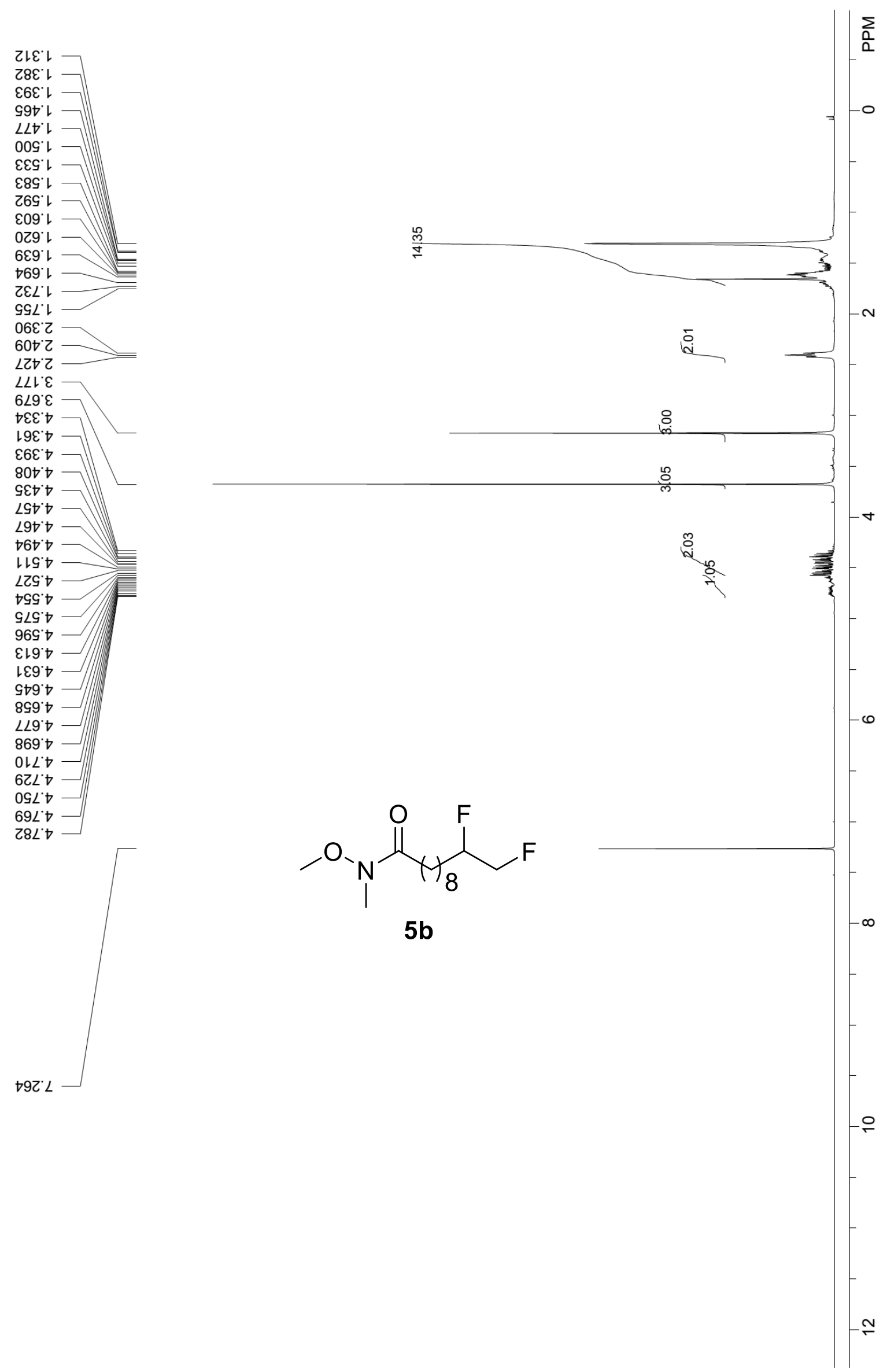


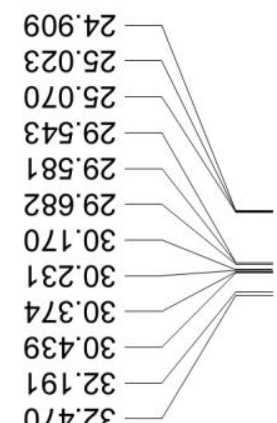

629.19

$9 \triangleright 0^{\circ} L L$

$09 \varepsilon^{\circ} \angle L$

Z89'LL

乙६૬' '८

$9 S L \cdot \varepsilon 8$

9९乙'ง8

$\varepsilon 8 t^{\circ} 98$

ฤ乙้เ6

ยEฑ เ6

$\angle 966^{\circ} \mathrm{C}$

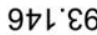

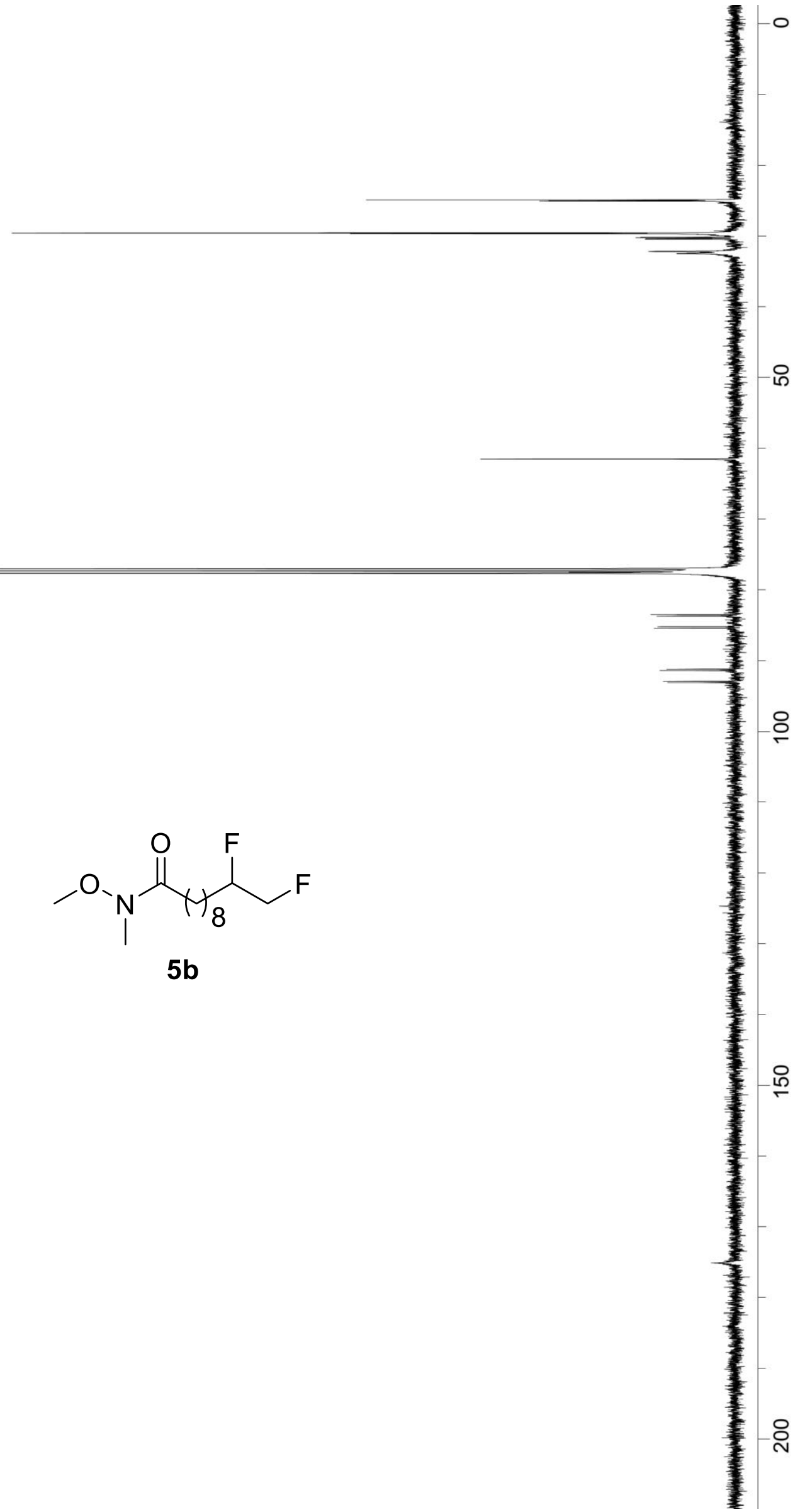

$9 เ \operatorname{LSLL}^{\circ}$ 
I8 $676-7$

LL'676-

$98 \cdot 88 \mathrm{I}->$
$\mathrm{I} 8^{\circ} \cdot 88 \mathrm{I}-$

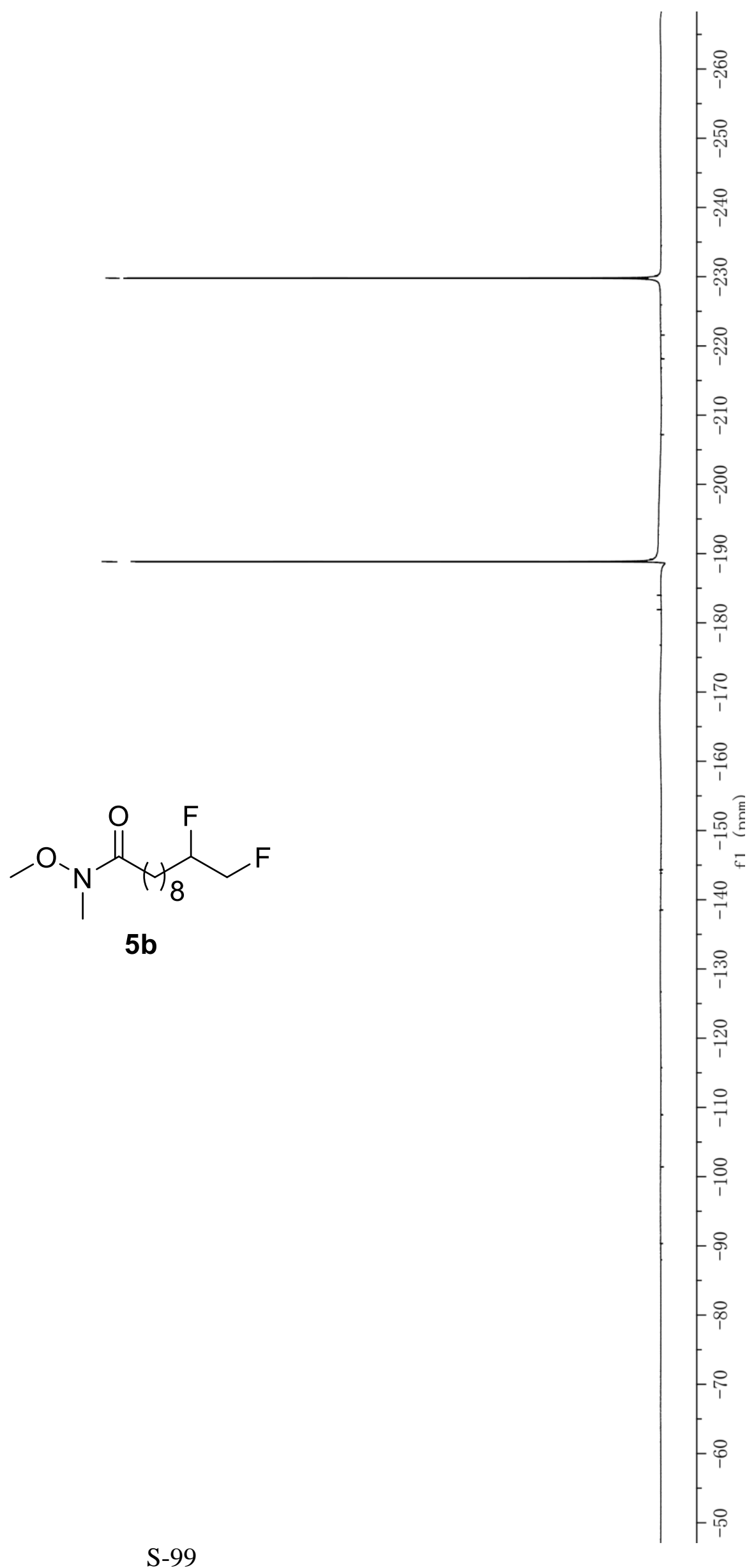



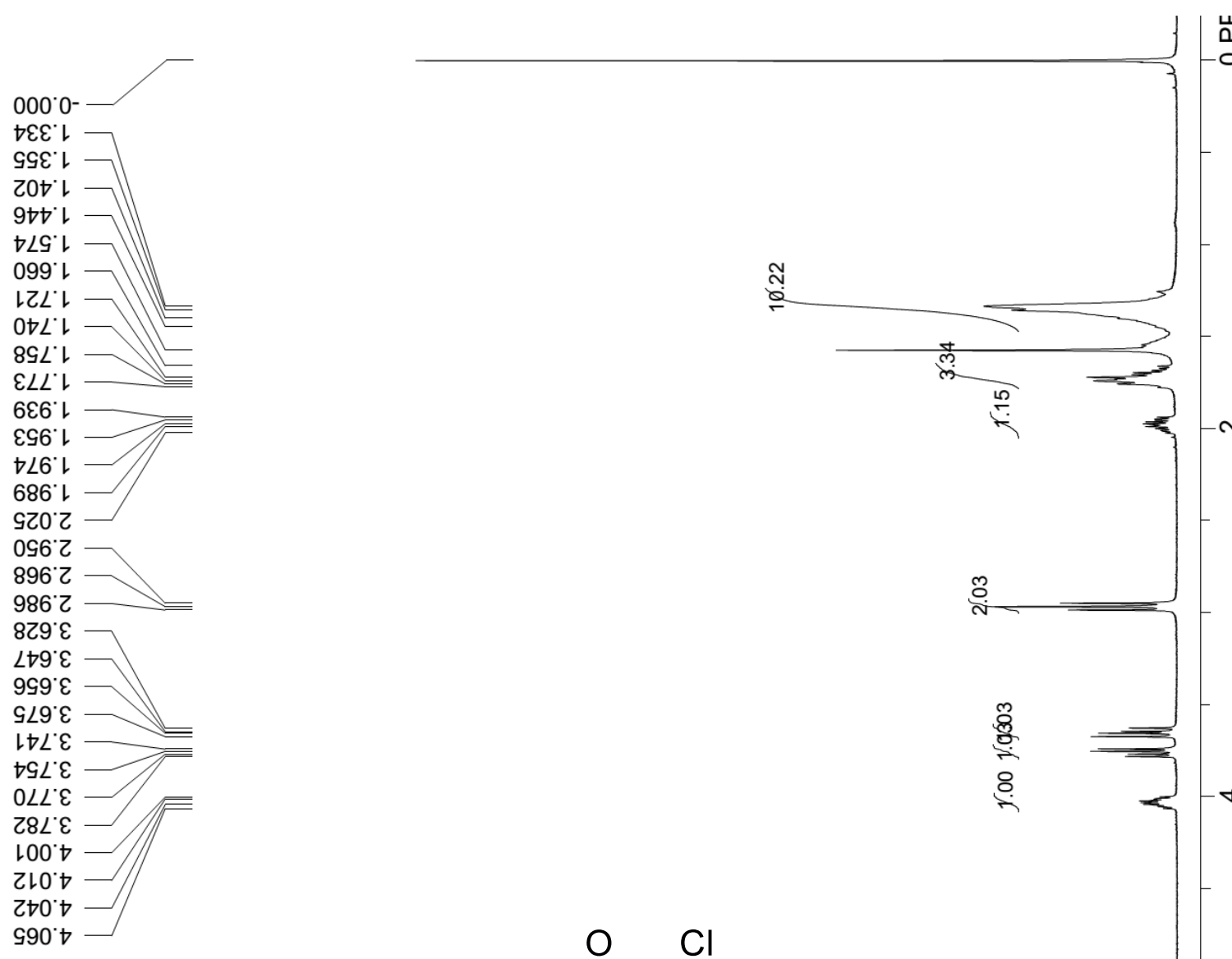<smiles>CC(CCl)NC(=O)c1ccccc1</smiles>

$6 a$

$6 \& 9^{\circ} L$

${ }^{89} \mathrm{~S}^{\circ} \mathrm{L}$

9L9' $L$

$6+6.2$

\&96. $L$

L $\angle 66^{\circ} L$

$\varepsilon \angle 6^{\circ} L$ 
$819^{\circ} \circ 2$

SOL.9Z

GटZ 62

$\varepsilon \nabla S^{\prime} 62$

$\varepsilon 0962$

$899^{\circ} 62$

乙๕६' $\varsigma \varepsilon$

$206.8 \varepsilon$

00987

$\varepsilon \angle G^{\prime} \vdash 9$

$S+0 . \angle L$

$09 \varepsilon^{\circ} L L$

$09 \varepsilon^{\circ} \angle L$
$189^{\circ} L L$

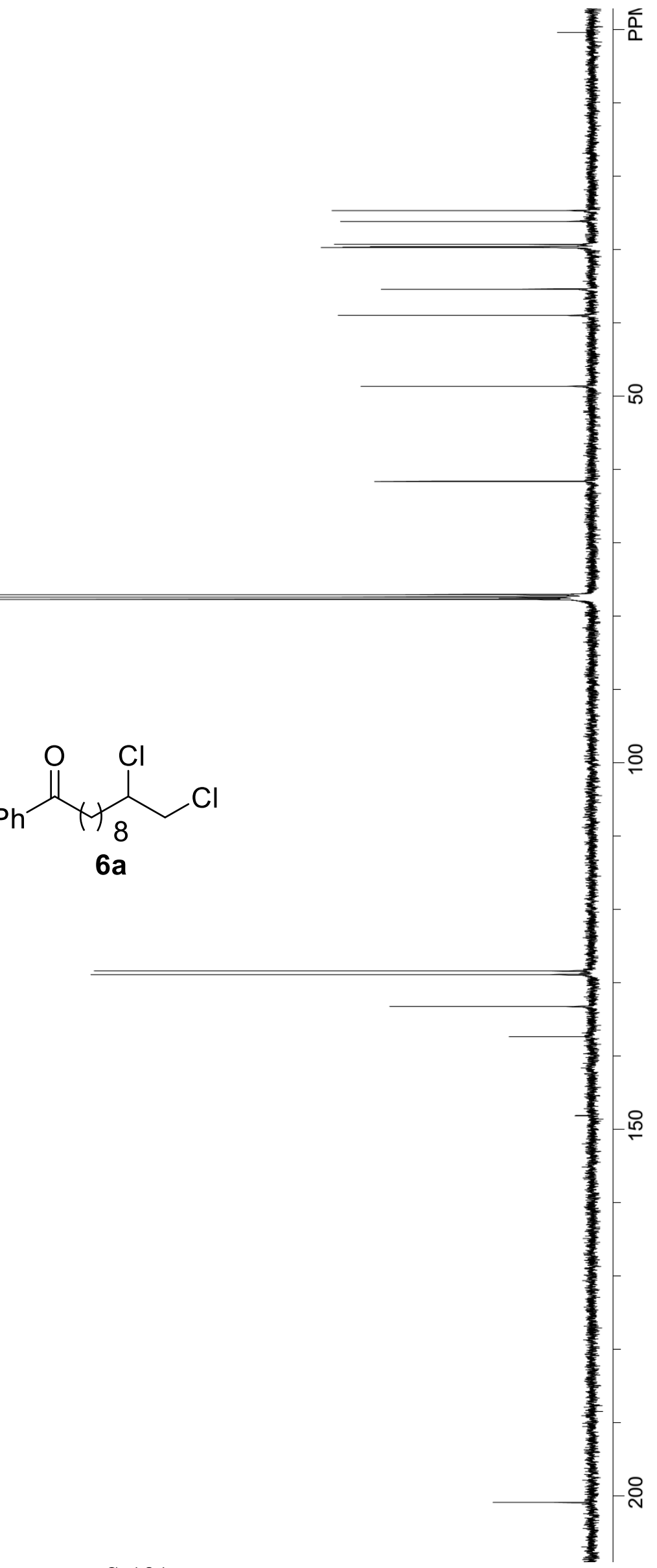

$98 \varepsilon^{\circ} 8 Z \mathrm{~L}$

$968.8 Z L$

८ย乙 $\varepsilon \varepsilon\llcorner$

$68 \varepsilon^{\circ} \angle \mathcal{L}$

$1 Z 6^{\circ} 00 Z$

S-101 

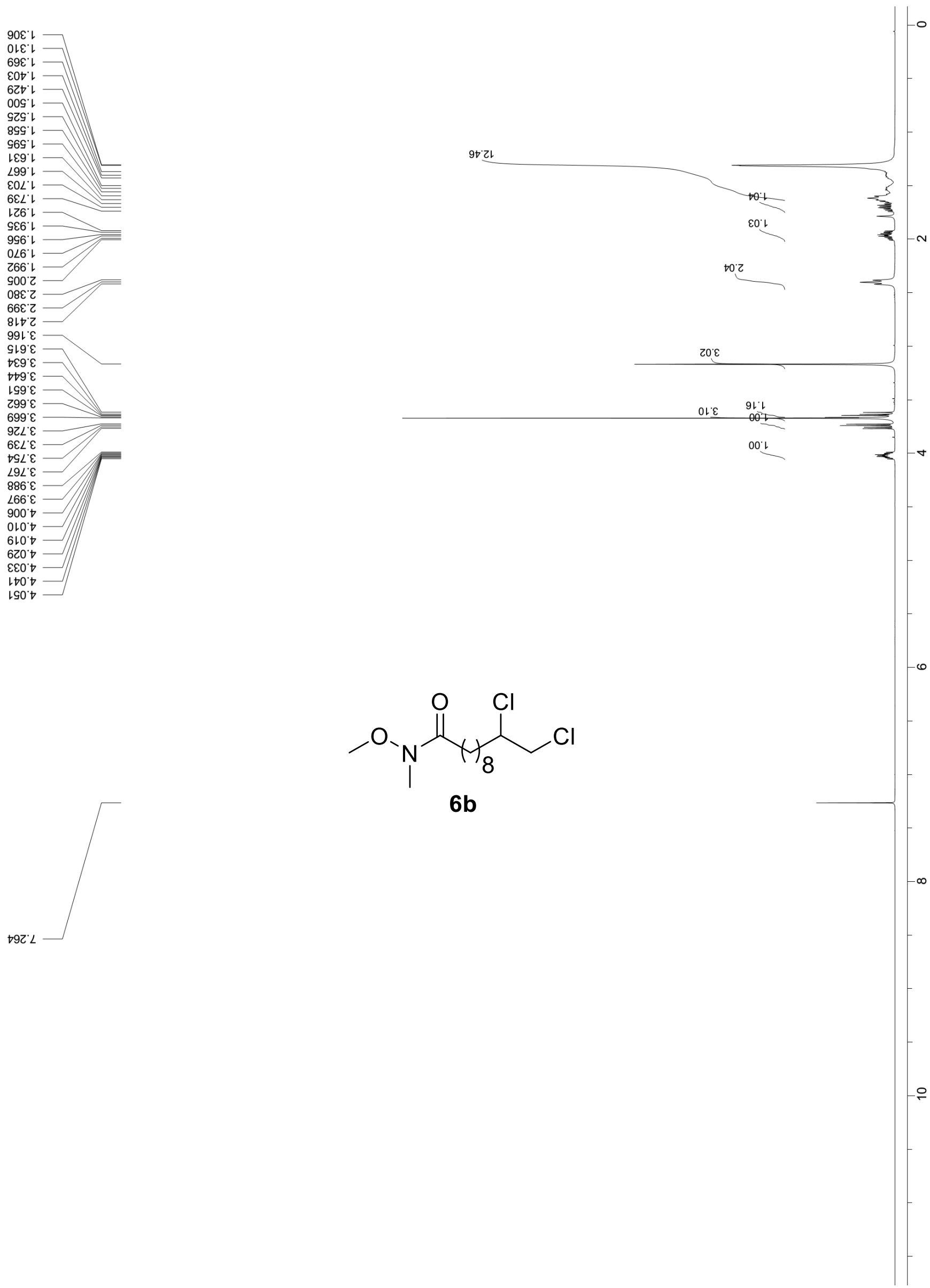

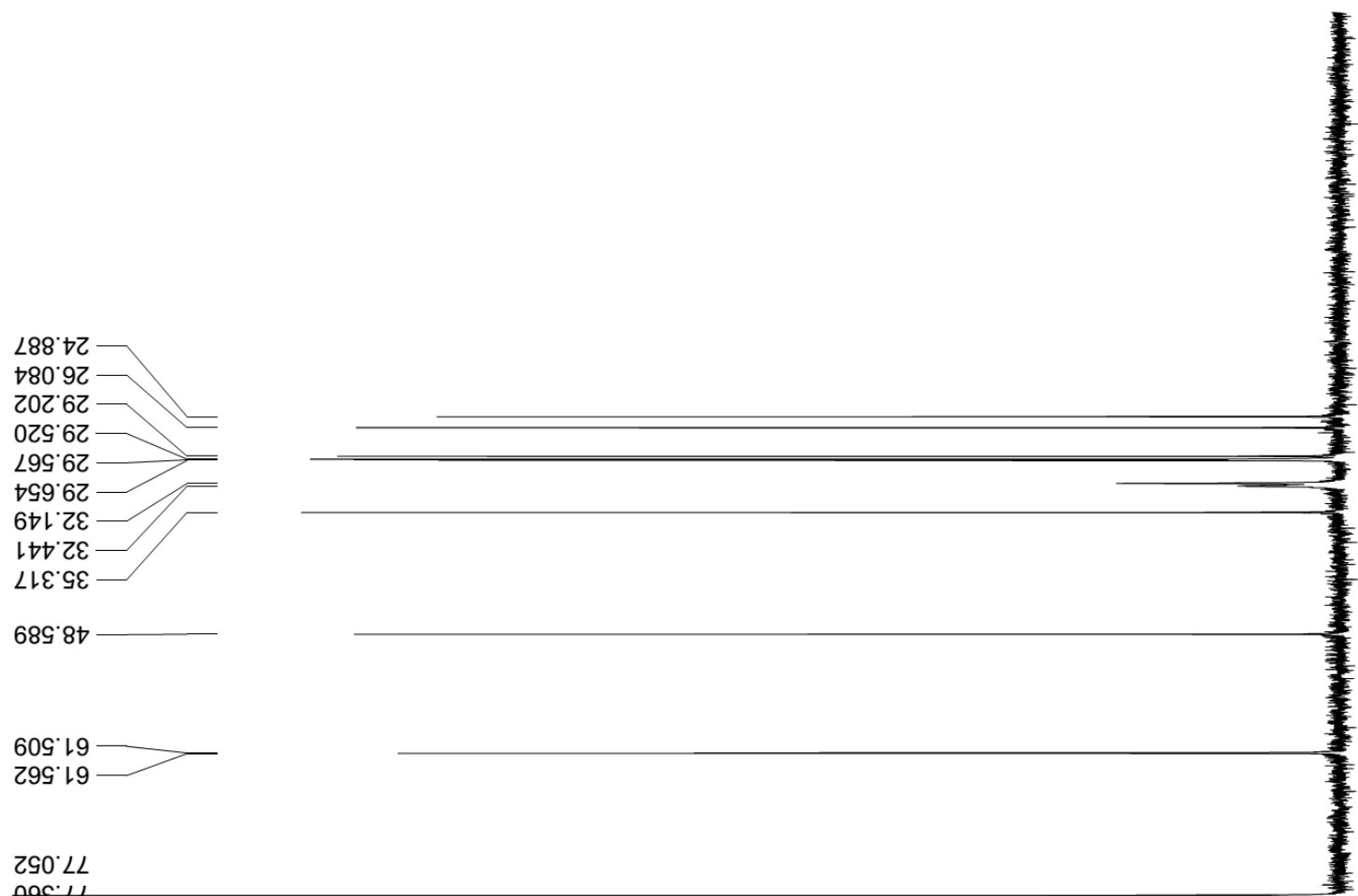

$290^{\circ} \mathrm{LL}$

थव: $\angle 1$

$989^{\circ} \mathrm{LL}$<smiles>CON(C)C(=O)[18CH]C(Cl)CCl</smiles>

$6 b$

L60'SLL 